\title{
Room Temperature Hydroamination of Alkenyl Ureas Catalyzed by a \\ Gold(I) Carbene Complex
}

\author{
Christopher F. Bender and Ross A. Widenhoefer* \\ P. M. Gross Chemical Laboratory \\ Duke University
}

Durham, North Carolina 27708-0346

\section{Supporting Information}

Experimental procedures, analytical and spectroscopic data, and copies of NMR spectra for heterocycles and selected compounds (54 pages). 


\section{Experimental}

General Methods. Reactions were performed under a nitrogen atmosphere utilizing standard Shlenk and drybox techniques unless specified otherwise. NMR were obtained on a Varian spectrometer operating at $400 \mathrm{MHz}$ for ${ }^{1} \mathrm{H} \mathrm{NMR}$ and $100 \mathrm{MHz}$ for ${ }^{13} \mathrm{C} \mathrm{NMR}$ in $\mathrm{CDCl}_{3}$ at $25{ }^{\circ} \mathrm{C}$ unless stated otherwise. IR spectra were obtained on a Nicolet Avatar 360-FT IR spectrometer. Gas chromatography was performed on a HP 5890 gas chromatography equipped with a 25 m polydimethylsiloxane capillary column. Column chromatography was performed employing 230-450 mesh silica gel (Sorbent Technologies). All compounds were isolated as colorless oils unless noted otherwise. Elemental analyses were performed by Complete Analysis Laboratories (Parsippany, NJ). Thin layer chromatography (TLC) was performed on silica gel $60 \mathrm{~F}_{254}$. Room temperature is $22-24{ }^{\circ} \mathrm{C}$.

1,4-Dioxane (anhydrous Acros), methanol (anhydrous Aldrich), $\mathrm{Au}\left(\mathrm{Me}_{2} \mathrm{~S}\right) \mathrm{Cl}$ (Aldrich), and 4 (Strem) were used as received. Tetrahydrofuran (THF) and diethyl ether were distilled from sodium benzophenone ketyl, and $\mathrm{CDCl}_{3}$ (Cambridge Isotope Labs) was distilled from CaH. 2,2-Diphenyl-4pentenylamine $\quad($ S1 $),{ }^{\text {S1 }} \quad C$-[1-(2-methylallyl)-cyclohexyl]methylamine $\quad\left(\right.$ S2),${ }^{\text {S1 }} \quad C$-(1-but-3-enylcyclohexyl)methylamine $\left(\right.$ S3) ${ }^{\text {S1 }} \quad$ 2-isopropyl-4-pentenylamine $\quad(\text { S4 })^{\text {S1 }} \quad$ 2,2-dimethyl-4-pentenenitrile (S5), ${ }^{\mathrm{S} 1}$ methyl-2-phenyl-4-pentenoate (S6), ${ }^{\mathrm{S} 2}$ benzyl 2,2-diphenyl-4-pentenylcarbamate (2a) ${ }^{\mathrm{S} 3} \mathrm{~N}-(2,2-$ diphenyl-4-pentenyl)acetamide (2b) ${ }^{\mathrm{S} 4} \mathrm{~N}$-(2,2-diphenyl-4-pentenyl)- $N^{\prime}$-phenylurea (2c) ${ }^{\mathrm{S} 4}$ and $\mathrm{Au}(\mathbf{4}) \mathrm{Cl}^{\mathrm{S} 5}$ were synthesized employing published procedures. 


\section{Substrates}

$N$-(2,2-Diphenyl-4-pentenyl)- $N^{\prime}$-phenylurea (2c). Phenylisocyanate $(0.31 \mathrm{~mL}, 2.8 \mathrm{mmol})$ was added dropwise to a solution of $\mathbf{S} 1(0.66 \mathrm{~g}, 2.8 \mathrm{mmol})$ in $\mathrm{THF}(10 \mathrm{~mL})$ at $0{ }^{\circ} \mathrm{C}$ and the reaction mixture was stirred overnight. The resulting solution was diluted with ether $(50 \mathrm{~mL})$, washed with $1 \mathrm{M} \mathrm{HCl}(25$ $\mathrm{mL})$, sat. $\mathrm{NaHCO}_{3}(25 \mathrm{~mL})$, and brine $(25 \mathrm{~mL})$, dried $\left(\mathrm{MgSO}_{4}\right)$, and concentrated. The resulting white solid was chromatographed (ether- $\left.\mathrm{CH}_{2} \mathrm{Cl}_{2}=1: 9\right)$ to give $2 \mathrm{c}(0.79 \mathrm{~g}, 80 \%)$ as a white solid. mp 171$172.5{ }^{\circ} \mathrm{C} . \quad \mathrm{TLC}\left(\mathrm{ether}-\mathrm{CH}_{2} \mathrm{Cl}_{2}=1: 9\right): R_{f}=0.64 .{ }^{1} \mathrm{H}$ NMR: $\delta \quad 6.99-7.24(\mathrm{~m}, 15 \mathrm{H}), 6.56(\mathrm{br}, 1 \mathrm{H})$, $5.40(\mathrm{tdd}, J=7.2,10.1,17.1 \mathrm{~Hz}, 1 \mathrm{H}), 4.89-4.96(\mathrm{~m}, 2 \mathrm{H}), 4.55(\mathrm{t}, J=5.6 \mathrm{~Hz}, 1 \mathrm{H}), 3.90(\mathrm{~d}, J=5.6 \mathrm{~Hz}$ $2 \mathrm{H}), 2.92(\mathrm{~d}, J=7.0 \mathrm{~Hz}, 2 \mathrm{H}) .{ }^{13} \mathrm{C}\left\{{ }^{1} \mathrm{H}\right\}$ NMR: $\delta \quad 155.9,145.5,138.4,133.9,129.3,128.1,126.5$, 124.0, 121.5, 118.7, 50.3, 47.1, 42.0. IR (neat, $\mathrm{cm}^{-1}$ ): 3324, 2360, 1642, 1550, 1232, 694. HRMS calcd (found) for $\mathrm{C}_{24} \mathrm{H}_{25} \mathrm{~N}_{2} \mathrm{O}\left(\mathrm{MH}^{+}\right): 357.1967$ (357.1966).

$N$-(2,2-Diphenyl-4-pentenyl)- $N$ '-ethylurea $\quad$ (S7), $\quad N$-(4-bromophenyl)- $N^{\prime}$-(2,2-diphenyl-4pentenyl)urea (S8), $N$-(2,2-diphenyl-4-pentenyl)- $N^{\prime}$-(4-methoxyphenyl)urea (S9), $N$-(4-acetylphenyl)- $N{ }^{\prime}$ (2,2-diphenyl-4-pentenyl)urea (S10), $N$-[1-(2-methylallyl)cyclohexylmethyl]- $N$ '-phenylurea (S11), $N$-(1but-3-enylcyclohexylmethyl)- $N$ '-phenylurea $\quad$ (S12), $\quad N$-(1-but-3-enylcyclohexylmethyl)- $N$ '-ethylurea (S13), and $N$-(2-isopropyl-4-pentenyl)- $N$ '-phenylurea (S14) were synthesized employing a procedure similar to that used to synthesize $\mathbf{2 c}$.

S7. White solid, 57\%. mp 179.5-180 ${ }^{\circ} \mathrm{C}$. TLC (ether- $\left.\mathrm{CH}_{2} \mathrm{Cl}_{2}=1: 2\right): R_{f}=0.49 .{ }^{1} \mathrm{H}$ NMR: $\delta$ 7.17-7.31 (m, $10 \mathrm{H}), 5.45(\mathrm{tdd}, J=7.2,10.1,17.1 \mathrm{~Hz}, 1 \mathrm{H}), 4.95-5.02(\mathrm{~m}, 2 \mathrm{H}), 4.11$ (br t, $J=5.6 \mathrm{~Hz}, 1$ $\mathrm{H}), 3.88(\mathrm{~s}, 3 \mathrm{H}), 3.00-3.06(\mathrm{~m}, 2 \mathrm{H}), 2.88(\mathrm{~d}, J=7.0 \mathrm{~Hz}, 2 \mathrm{H}), 1.02(\mathrm{t}, J=7.2 \mathrm{~Hz}, 3 \mathrm{H}) .{ }^{13} \mathrm{C}\left\{{ }^{1} \mathrm{H}\right\}$ NMR: $\delta 158.1,145.7,134.1,128.4,128.2,126.5,118.6,50.5,47.3,41.9,35.4,15.5$. IR (neat, $\left.\mathrm{cm}^{-1}\right)$ : 3347, 3290, 1615, 1574, 1494, 697. Anal. calcd (found) for $\mathrm{C}_{20} \mathrm{H}_{24} \mathrm{~N}_{2} \mathrm{O}: \mathrm{H}, 7.84$ (7.84); C, 77.89 (78.00). HRMS calcd (found) for $\mathrm{C}_{20} \mathrm{H}_{24} \mathrm{~N}_{2} \mathrm{O}\left(\mathrm{M}^{+}\right)$: 308.1889 (308.1886). 
S8. White solid, $68 \% . \mathrm{mp} 219-220{ }^{\circ} \mathrm{C} . \quad \mathrm{TLC}\left(\right.$ ether- $\left.\mathrm{CH}_{2} \mathrm{Cl}_{2}=1: 19\right): R_{f}=0.53 .{ }^{1} \mathrm{H}$ NMR $\left(d_{6^{-}}\right.$ DMSO): $\delta 8.69$ (br s, $1 \mathrm{H}), 7.19-7.36(\mathrm{~m}, 14 \mathrm{H}), 5.56$ (br t, $J=5.8 \mathrm{~Hz}, 1 \mathrm{H}), 5.45$ (tdd, $J=7.0,12.3$, $17.1 \mathrm{~Hz}, 1 \mathrm{H}), 4.90-4.99(\mathrm{~m}, 2 \mathrm{H}), 3.90(\mathrm{~d}, J=5.6 \mathrm{~Hz}, 2 \mathrm{H}), 2.88(\mathrm{~d}, J=7.0 \mathrm{~Hz}, 2 \mathrm{H}) .{ }^{13} \mathrm{C}\left\{{ }^{1} \mathrm{H}\right\} \mathrm{NMR}$ $\left(d_{6}\right.$-DMSO): $\delta 154.8,145.7,139.8,134.3,131.4,128.1,127.7,126.0,119.3,118.1,112.2,49.7,45.4$ 40.9. IR (neat, $\mathrm{cm}^{-1}$ ): $3337,1647,1549,1489,1233,697$. Anal. calcd (found) for $\mathrm{C}_{24} \mathrm{H}_{23} \mathrm{BrN}_{2} \mathrm{O}: \mathrm{H}$, 5.32 (5.23); C, 66.21 (66.07). HRMS calcd for $\mathrm{C}_{24} \mathrm{H}_{23}{ }^{79} \mathrm{BrN}_{2} \mathrm{O}\left(\mathrm{M}^{+}\right)$: 434.0994 (434.0994).

S9. White solid, $48 \% . \mathrm{mp} 177-177.5{ }^{\circ} \mathrm{C} . \quad \mathrm{TLC}\left(\right.$ ether- $\left.\mathrm{CH}_{2} \mathrm{Cl}_{2}=1: 9\right): R_{f}=0.38 .{ }^{1} \mathrm{H}$ NMR: $\delta$ 7.09-7.26 (m, $10 \mathrm{H}), 6.92-6.95$ (m, 2 H), 6.73-6.77 (m, 2 H), 6.14 (br s, $1 \mathrm{H}), 5.42$ (tdd, $J=7.2,10.1$, $17.1 \mathrm{~Hz}, 1 \mathrm{H}), 4.92-4.99(\mathrm{~m}, 2 \mathrm{H}), 4.29$ (br t, $J=5.5 \mathrm{~Hz}, 1 \mathrm{H}), 3.91$ (d, $J=5.6 \mathrm{~Hz}, 2 \mathrm{H}), 3.79(\mathrm{~s}, 3 \mathrm{H})$, $2.82(\mathrm{~d}, J=7.0 \mathrm{~Hz}, 2 \mathrm{H}) .{ }^{13} \mathrm{C}\left\{{ }^{1} \mathrm{H}\right\}$ NMR: $\delta \quad 157.2,156.6,145.5,133.9,130.6,128.4,128.1,126.5$, 125.4, 118.7, 114.7, 55.6, 50.5, 47.1, 42.1. IR (neat, $\mathrm{cm}^{-1}$ ): 3331, 1642, 1554, 1508, 1235, 696. Anal. calcd (found) for $\mathrm{C}_{25} \mathrm{H}_{26} \mathrm{~N}_{2} \mathrm{O}_{2}$ : H, 6.78 (6.75); C, 77.69 (77.74). HRMS calcd (found) for $\mathrm{C}_{25} \mathrm{H}_{26} \mathrm{~N}_{2} \mathrm{O}_{2}$ $\left(\mathrm{M}^{+}\right): 386.1994$ (386.1989).

S10. White, waxy solid, 88\%. TLC (ether- $\left.\mathrm{CH}_{2} \mathrm{Cl}_{2}=1: 9\right): R_{f}=0.39 .{ }^{1} \mathrm{H}$ NMR: $\delta$ 7.76-7.79 (m, 2 H), 7.44 (br s, $1 \mathrm{H}), 7.14-7.30(\mathrm{~m}, 12 \mathrm{H}), 5.42(\mathrm{tdd}, J=7.0,10.1,17.1 \mathrm{~Hz}, 1 \mathrm{H}), 4.93-5.00$ (m, 2 H), 4.86 (br t, $J=5.6 \mathrm{~Hz}, 1 \mathrm{H}), 3.97(\mathrm{~d}, J=5.8 \mathrm{~Hz}, 2 \mathrm{H}), 2.87$ (d, $J=7.0 \mathrm{~Hz}, 2 \mathrm{H}), 2.47(\mathrm{~s}, 3 \mathrm{H})$. ${ }^{13} \mathrm{C}\left\{{ }^{1} \mathrm{H}\right\}$ NMR: $\delta 197.8,155.0,145.4,144.2,133.8,131.2,130.1,128.4,128.1,126.6,118.8,118.0$, 50.2, 47.0, 42.0, 26.5. IR (neat, $\mathrm{cm}^{-1}$ ): 3343, 1660, 1533, 1223, 1176, 699. Anal. calcd (found) for $\mathrm{C}_{26} \mathrm{H}_{26} \mathrm{~N}_{2} \mathrm{O}_{2}$ : H, 6.58 (6.54); C, 78.36 (78.56). HRMS calcd (found) for $\mathrm{C}_{26} \mathrm{H}_{26} \mathrm{~N}_{2} \mathrm{O}_{2}\left(\mathrm{M}^{+}\right)$: 398.1994 , (398.2002).

S11. White solid, $85 \%$. TLC (ether- $\left.\mathrm{CH}_{2} \mathrm{Cl}_{2}=1: 19\right): R_{f}=0.41 .{ }^{1} \mathrm{H}$ NMR: $\delta 7.86(\mathrm{~s}, 1 \mathrm{H}), 7.31$ $(\mathrm{d}, J=8.4 \mathrm{~Hz}, 2 \mathrm{H}), 7.22(\mathrm{t}, J=7.8 \mathrm{~Hz}, 2 \mathrm{H}), 6.99(\mathrm{t}, J=7.2 \mathrm{~Hz}, 1 \mathrm{H}), 5.81(\mathrm{br} \mathrm{t}, J=5.6 \mathrm{~Hz}, 1 \mathrm{H}), 4.78$ (s, $1 \mathrm{H}), 4.61(\mathrm{~s}, 1 \mathrm{H}), 3.20(\mathrm{~d}, J=6.0 \mathrm{~Hz}, 2 \mathrm{H}), 1.99(\mathrm{~s}, 2 \mathrm{H}), 1.72(\mathrm{~s}, 3 \mathrm{H}), 1.31-1.44(\mathrm{~m}, 10 \mathrm{H})$. 
${ }^{13} \mathrm{C}\left\{{ }^{1} \mathrm{H}\right\}$ NMR: $\delta \quad 157.1,143.1,139.3,129.1,123.1,120.4,114.9,46.4,44.4,37.6,33.9,26.2,25.5$, 21.8. IR (neat, $\mathrm{cm}^{-1}$ ): $3361,2928,1642,1559,1236,893 . \mathrm{mp} 99.5-100.5^{\circ} \mathrm{C}$. Anal. calcd (found) for $\mathrm{C}_{18} \mathrm{H}_{26} \mathrm{~N}_{2} \mathrm{O}$ : H, 9.15 (9.11); C, 75.48 (75.45). HRMS calcd (found) for $\mathrm{C}_{18} \mathrm{H}_{26} \mathrm{~N}_{2} \mathrm{O}\left(\mathrm{M}^{+}\right)$: 286.2045, (286.2051).

S12. White solid, 83\%. TLC (ether- $\left.\mathrm{CH}_{2} \mathrm{Cl}_{2}=1: 9\right): R_{f}=0.65 .{ }^{1} \mathrm{H}$ NMR: $\delta 7.93($ br s, $1 \mathrm{H}$ ), $7.30(\mathrm{~d}, J=7.9 \mathrm{~Hz}, 2 \mathrm{H}), 7.21(\mathrm{~d}, J=7.6 \mathrm{~Hz}, 2 \mathrm{H}), 6.97(\mathrm{t}, J=6.7 \mathrm{~Hz}, 1 \mathrm{H}), 5.87$ (br t, $J=5.6 \mathrm{~Hz}, 1$ H), $5.74(\operatorname{tdd}, J=6.5,10.3,16.9 \mathrm{~Hz}, 1 \mathrm{H}), 4.86-4.98(\mathrm{~m}, 2 \mathrm{H}), 3.13(\mathrm{~d}, J=6.0 \mathrm{~Hz}, 2 \mathrm{H}), 1.90-1.96(\mathrm{~m}, 2$ $\mathrm{H}), 1.20-1.44(\mathrm{~m}, 12 \mathrm{H}) .{ }^{13} \mathrm{C}\left\{{ }^{1} \mathrm{H}\right\} \mathrm{NMR}: \delta 157.1,139.4,139.4,129.0,122.9,120.1,114.2,46.4,36.4$, 34.7, 33.5, 27.5, 26.3, 21.5. IR (neat, $\mathrm{cm}^{-1}$ ): $3333,2925,1645,1557,1233$, 694. Anal. calcd (found) for $\mathrm{C}_{18} \mathrm{H}_{26} \mathrm{~N}_{2} \mathrm{O}:$ H, 9.15 (9.29); C, 75.48 (75.32). HRMS calcd (found) for $\mathrm{C}_{18} \mathrm{H}_{27} \mathrm{~N}_{2} \mathrm{O}\left(\mathrm{M}^{+}\right.$): 287.2123 (287.2119).

S13. Colorless oil, 99\%. TLC (ether- $\left.\mathrm{CH}_{2} \mathrm{Cl}_{2}=1: 2\right): R_{f}=0.51 .{ }^{1} \mathrm{H}$ NMR: $\delta 5.79$ (tdd, $J=6.5$, 10.3, 17.1 Hz, $1 \mathrm{H}), 4.89-5.01(\mathrm{~m}, 2 \mathrm{H}), 4.83$ (br t, $J=5.1 \mathrm{~Hz}, 1 \mathrm{H}), 4.71$ (br t, $J=5.5 \mathrm{~Hz}, 1 \mathrm{H}), 3.18$ $(\mathrm{dq}, J=5.6,7.2 \mathrm{~Hz}, 2 \mathrm{H}), 3.07(\mathrm{~d}, J=6.0 \mathrm{~Hz}, 2 \mathrm{H}), 1.93-1.99(\mathrm{~m}, 2 \mathrm{H}), 1.23-1.48(\mathrm{~m}, 12 \mathrm{H}), 1.11(\mathrm{t}, J$ $=7.2 \mathrm{~Hz}, 3 \mathrm{H}) .{ }^{13} \mathrm{C}\left\{{ }^{1} \mathrm{H}\right\}$ NMR: $\delta \quad 158.9,139.6,114.2,46.5,36.3,35.4,34.9,33.7,27.5,26.4,21.6$, 15.7. IR (neat, $\mathrm{cm}^{-1}$ ): $3353,2924,2857,1629,1568,1253$. Anal. calcd (found) for $\mathrm{C}_{14} \mathrm{H}_{26} \mathrm{~N}_{2} \mathrm{O}: \mathrm{H}$, 10.99 (11.10); C, 70.54 (70.52). HRMS calcd (found) for $\mathrm{C}_{14} \mathrm{H}_{26} \mathrm{~N}_{2} \mathrm{O}\left(\mathrm{M}^{+}\right)$: 238.2045 (238.2047).

S14. White solid, 38\%. TLC (EtOAc-hexanes $=1: 2): R_{f}=0.29 .{ }^{1} \mathrm{H}$ NMR $(300 \mathrm{MHz}): \delta 7.68$ (br s, 1 H), 7.20-7.32 (m, 4 H), 6.96-7.02 (m, 1 H), 5.64-5.78 (m, 2 H), 4.91-5.01 (m, 2 H), 3.08-3.26 (m, $2 \mathrm{H}), 2.04-2.13(\mathrm{~m}, 1 \mathrm{H}), 1.88-1.98(\mathrm{~m}, 1 \mathrm{H}), 1.63-1.76(\mathrm{~m}, 1 \mathrm{H}), 1.34-1.44(\mathrm{~m}, 1 \mathrm{H}), 0.85(\mathrm{~d}, J=$ $6.9 \mathrm{~Hz}, 3 \mathrm{H}), 0.84(\mathrm{~d}, J=6.9 \mathrm{~Hz}, 3 \mathrm{H}) .{ }^{13} \mathrm{C}\left\{{ }^{1} \mathrm{H}\right\} \mathrm{NMR}: \delta \quad 156.9,139.4,137.5,129.0,122.9,120.0$, 116.1, 44.3, 41.0, 33.5, 28.3, 19.6, 19.1. IR (neat, $\mathrm{cm}^{-1}$ ): 3358, 2958, 1642, 1555, 1241, 694. Anal. 
calcd (found) for $\mathrm{C}_{15} \mathrm{H}_{22} \mathrm{~N}_{2} \mathrm{O}: \mathrm{H}, 9.00$ (9.07); C, 73.13 (73.27). HRMS calcd (found) for $\mathrm{C}_{15} \mathrm{H}_{22} \mathrm{~N}_{2} \mathrm{O}$ $\left(\mathrm{M}^{+}\right): 246.1732(246.1731)$.

$N$-(2,2-Dimethyl-1-phenyl-4-pentenyl)- $N$ '-phenylurea (S15). A solution of phenyl magnesium bromide (1 M in THF, $15 \mathrm{~mL}, 15 \mathrm{mmol})$ and $\mathbf{S 5}(1.08 \mathrm{~g}, 9.89 \mathrm{mmol})$ in ether $(60 \mathrm{~mL})$ was refluxed for $17 \mathrm{~h}$. The resulting suspension was cooled to $-15^{\circ} \mathrm{C}$, treated with methanol $(17 \mathrm{~mL})$, stirred for $5 \mathrm{~min}$, cooled to $-78{ }^{\circ} \mathrm{C}$, and treated with $\mathrm{NaBH}_{4}(920 \mathrm{mg}, 24.3 \mathrm{mmol})$ added in one portion. The reaction mixture was warmed slowly to room temperature and stirred for an additional $1 \mathrm{~h}$. The resulting suspension was treated with $1 \mathrm{M} \mathrm{NaOH}(50 \mathrm{~mL})$ and extracted with ether $(2 \times 50 \mathrm{~mL})$. The combined organic extracts were washed aqueous sodium hydroxide $(1 \mathrm{M}, 5 \mathrm{~mL})$ and brine $(45 \mathrm{~mL})$, dried $\left(\mathrm{MgSO}_{4}\right)$, and concentrated to yield 2,2-dimethyl-1-phenyl-4-pentenylamine (S16, $760 \mathrm{mg}, 41 \%, 93 \%$ pure) that was used in the subsequent step without further purification.

Phenylisocyanate $(210 \mu \mathrm{L}, 1.93 \mathrm{mmol})$ was added dropwise to a solution of S16 (380 $\mathrm{mg}, 2.01$ mmol) in THF $(10 \mathrm{~mL})$ at $0{ }^{\circ} \mathrm{C}$ and the reaction was stirred overnight. The resulting solution was diluted with ether $(25 \mathrm{~mL})$, washed with $1 \mathrm{M} \mathrm{HCl}(15 \mathrm{~mL})$, sat. aqueous $\mathrm{NaHCO}_{3}(15 \mathrm{~mL})$, and brine (15 mL), dried $\left(\mathrm{MgSO}_{4}\right)$, and concentrated. The resulting residue was chromatographed (ether- $\mathrm{CH}_{2} \mathrm{Cl}_{2}$ $=1: 30)$ to give $\mathbf{S 1 5}(450 \mathrm{mg}, 76 \%)$ as a white solid. TLC (ether- $\left.\mathrm{CH}_{2} \mathrm{Cl}_{2}=1: 30\right): R_{f}=0.45 .{ }^{1} \mathrm{H}$ NMR: ઈ 7.73 (br s, $1 \mathrm{H}), 7.14-7.19(\mathrm{~m}, 9 \mathrm{H}), 6.95-7.01(\mathrm{~m}, 1 \mathrm{H}), 6.48$ (br s, $1 \mathrm{H}), 5.81$ (tdd, $J=7.3,10.1,17.3$ Hz, $1 \mathrm{H}), 4.98-5.05(\mathrm{~m}, 2 \mathrm{H}), 4.72(\mathrm{~d}, J=8.7 \mathrm{~Hz}, 1 \mathrm{H}), 1.92-2.05(\mathrm{~m}, 2 \mathrm{H}), 0.82(\mathrm{~s}, 3 \mathrm{H}), 0.78$ (s, $3 \mathrm{H})$. ${ }^{13} \mathrm{C}\left\{{ }^{1} \mathrm{H}\right\}$ NMR: $\delta \quad 156.3,140.4,139.1,134.8,129.1,128.5,127.9,127.1,123.1,120.2,118.1,62.0$, 44.2, 37.8, 24.1, 23.5. IR (neat, $\mathrm{cm}^{-1}$ ): 3345, 1642, 1549, 1498, 1234, 696. Anal. calcd (found) for $\mathrm{C}_{20} \mathrm{H}_{24} \mathrm{~N}_{2} \mathrm{O}$ : H, 7.84 (7.86); C, 77.89 (77.78). HRMS calcd (found) for $\mathrm{C}_{20} \mathrm{H}_{24} \mathrm{~N}_{2} \mathrm{O}\left(\mathrm{M}^{+}\right)$: 308.1889 (308.1888). 
$N$-(2,2-Dimethyl-1-phenyl-4-pentenyl)- $N^{\prime}$-ethylurea (S17). S17 was synthesized in 66\% yield as a colorless oil that solidified to a white solid over several days employing a procedure similar to that used to synthesize S15. TLC (ether- $\left.\mathrm{CH}_{2} \mathrm{Cl}_{2}=1: 9\right): R_{f}=0.22 .{ }^{1} \mathrm{H}$ NMR: $\delta \quad 7.20-7.29(\mathrm{~m}, 5 \mathrm{H}), 5.78-$ $5.91(\mathrm{~m}, 2 \mathrm{H}), 4.95-5.10(\mathrm{~m}, 3 \mathrm{H}), 4.54(\mathrm{~d}, J=8.2 \mathrm{~Hz}, 1 \mathrm{H}), 3.03-3.16(\mathrm{~m}, 2 \mathrm{H}), 1.98-2.11(\mathrm{~m}, 2 \mathrm{H})$, $0.96(\mathrm{t}, J=7.2 \mathrm{~Hz}, 3 \mathrm{H}), 0.87(\mathrm{~s}, 3 \mathrm{H}), 0.83(\mathrm{~s}, 3 \mathrm{H}) .{ }^{13} \mathrm{C}\left\{{ }^{1} \mathrm{H}\right\} \mathrm{NMR}: \delta \quad 158.5,140.8,135.0,128.5$, $127.8,127.0,117.9,62.2,44.2,37.9,35.2,24.2,23.4,15.5$. IR (neat, $\mathrm{cm}^{-1}$ ): 3348, 2969, 1628, 1559, 1252, 703. Anal. calcd (found) for $\mathrm{C}_{16} \mathrm{H}_{24} \mathrm{~N}_{2} \mathrm{O}: \mathrm{H}, 9.29$ (9.14); C, 73.81 (73.78). HRMS calcd (found) for $\mathrm{C}_{16} \mathrm{H}_{24} \mathrm{~N}_{2} \mathrm{O}\left(\mathrm{M}^{+}\right)$: 260.1889 (260.1889).

$\boldsymbol{N}$-Benzyl-N'-4-pentenylurea (S18). 4-Pentenitrile (4.38 g, $54.0 \mathrm{mmol})$ was added to a suspension of $\mathrm{LiAlH}_{4}(8.15 \mathrm{~g}, 215 \mathrm{mmol})$ in ether $(250 \mathrm{~mL})$ at $0{ }^{\circ} \mathrm{C}$. The reaction mixture was warmed to room temperature, stirred overnight, cooled to $0{ }^{\circ} \mathrm{C}$, and quenched by successive addition of water $(11.5 \mathrm{~mL}), 15 \% \mathrm{NaOH}(11.5 \mathrm{~mL})$, and water $(11.5 \mathrm{~mL})$. The resulting suspension was warmed to room temperature, filtered through Celite, and eluted with ether $(200 \mathrm{~mL})$. The resulting solution was carefully concentrated to yield 4-pentenylamine ${ }^{\mathrm{S} 6}(\mathbf{S 1 9}, 38 \% \mathrm{w} / \mathrm{w}$ in ether, $3.31 \mathrm{~g}, 72 \%)$. S18 was synthesized from $\mathbf{S 1 9}$ employing a procedure similar to that used to synthesize 2c.

For S19: ${ }^{1} \mathrm{H}$ NMR: $\delta 5.80(\mathrm{tdd}, J=6.5,10.3,17.1 \mathrm{~Hz}, 1 \mathrm{H}), 4.92-5.04(\mathrm{~m}, 2 \mathrm{H}), 2.70(\mathrm{t}, J=$ $7.1 \mathrm{~Hz}, 2 \mathrm{H}), 2.06-2.11(\mathrm{~m}, 2 \mathrm{H}), 1.50-1.57(\mathrm{~m}, 2 \mathrm{H}), 1.41$ (br. s, $2 \mathrm{H}) .{ }^{13} \mathrm{C}\left\{{ }^{1} \mathrm{H}\right\} \mathrm{NMR}: \delta \quad 138.5,114.8$ $41.8,32.8,31.2$

For S18: White solid, 70\%. TLC (ether $\left.-\mathrm{CH}_{2} \mathrm{Cl}_{2}=1: 2\right): R_{f}=0.60 .{ }^{1} \mathrm{H}$ NMR: $\delta \quad 7.18-7.27(\mathrm{~m}$, $5 \mathrm{H}), 5.73(\mathrm{tdd}, J=6.7,10.3,16.9 \mathrm{~Hz}, 1 \mathrm{H}), 5.63($ br t $, J=6.0 \mathrm{~Hz}, 1 \mathrm{H}), 5.33$ (br t, $J=5.8 \mathrm{~Hz}, 1 \mathrm{H})$, 4.91-4.99 (m, $2 \mathrm{H}), 4.22(\mathrm{~d}, J=5.8 \mathrm{~Hz}, 2 \mathrm{H}), 3.02-3.07(\mathrm{~m}, 2 \mathrm{H}), 1.96-2.01(\mathrm{~m}, 2 \mathrm{H}), 1.46$ (quintet, $J=$ $7.3 \mathrm{~Hz}, 2 \mathrm{H}) .{ }^{13} \mathrm{C}\left\{{ }^{1} \mathrm{H}\right\}$ NMR: $\delta 159.0,139.7,138.0,128.6,127.3,127.1,115.0,44.2,39.9,31.1,29.5$. 
IR (neat, $\mathrm{cm}^{-1}$ ): 3329, 2927, 1620, 1584, 1262, 692. Anal. calcd (found) for $\mathrm{C}_{13} \mathrm{H}_{18} \mathrm{~N}_{2} \mathrm{O}: \mathrm{H}, 8.31$ (8.40); C, 71.53 (71.18). HRMS calcd (found) for $\mathrm{C}_{13} \mathrm{H}_{18} \mathrm{~N}_{2} \mathrm{O}\left(\mathrm{M}^{+}\right)$: 218.1419 (218.1420).

$N$-Ethyl- $N^{\prime}$-(2-phenyl-4-pentenyl)urea (S21). S6 in THF (35 mL) was added to a solution of $\mathrm{KOH}(89 \%, 5.9 \mathrm{~g}, 94 \mathrm{mmol})$ in water $(25 \mathrm{~mL})$. $\mathrm{MeOH}(50 \mathrm{~mL})$ was added and the reaction mixture was heated at $40{ }^{\circ} \mathrm{C}$ for $19 \mathrm{~h}$. The resulting mixture was cooled to room temperature, acidified with concentrated $\mathrm{HCl}(10 \mathrm{~mL})$, and extracted with EtOAc $(3 \times 75 \mathrm{~mL})$. The combined organic extracts were washed with $1 \mathrm{M} \mathrm{HCl}(5 \mathrm{~mL})$ and brine $(45 \mathrm{~mL})$, dried $\left(\mathrm{MgSO}_{4}\right)$, and concentrated to yield 2-phenyl-4pentenoic acid $^{\mathrm{S} 2}(\mathbf{S 2 2}, 3.77 \mathrm{~g}, 92 \%)$ as a yellow oil.

Oxalyl chloride $(1.10 \mathrm{~mL}, 12.6 \mathrm{mmol})$ was added over $10 \mathrm{~min}$ to a solution of $\mathbf{S 2 2}(1.53 \mathrm{~g}, 8.68$ mmol) and toluene $(10 \mathrm{~mL})$, stirred for $3 \mathrm{~h}$, and concentrated to $\sim 5 \mathrm{~mL}$ under reduced pressure. The resulting solution was added over $15 \mathrm{~min}$ to a solution of ammonia in toluene $(10 \mathrm{~mL})$ at $-78{ }^{\circ} \mathrm{C}$. The reaction mixture was warmed to room temperature, stirred overnight, and concentrated under vacuum to give a pale yellow oil that was partitioned between $\mathrm{CH}_{2} \mathrm{Cl}_{2}(50 \mathrm{~mL})$ and $10 \% \mathrm{NaOH}(50 \mathrm{~mL})$. The layers were separated and the aqueous layer extracted with $\mathrm{CH}_{2} \mathrm{Cl}_{2}(3 \times 20 \mathrm{~mL})$. The combined organic extracts were washed with brine $(30 \mathrm{~mL})$, dried $\left(\mathrm{MgSO}_{4}\right)$ and concentrated. The resulting residue was chromatographed (EtOAc- $\left.\mathrm{CH}_{2} \mathrm{Cl}_{2}=1: 4\right)$ to give 2-phenyl-4-pentenamide ${ }^{\mathrm{S} 2}(\mathbf{S 2 3}, 1.05 \mathrm{~g}, 69 \%)$ as a white solid. $\mathrm{mp} 60-61{ }^{\circ} \mathrm{C}$.

A solution of $\mathbf{S 2 3}(653 \mathrm{mg}, 3.73 \mathrm{mmol})$ in ether $(10 \mathrm{~mL})$ was added to a suspension of $\mathrm{LiAlH}_{4}$ (580 $\mathrm{mg}, 15.3 \mathrm{mmol})$ in ether $(40 \mathrm{~mL})$ and the resulting suspension was refluxed for $16 \mathrm{~h}$. The reaction mixture was cooled to $0{ }^{\circ} \mathrm{C}$ and quenched by successive addition of water $(0.8 \mathrm{~mL}), 15 \% \mathrm{NaOH}(0.8$ $\mathrm{mL})$, and water $(0.8 \mathrm{~mL})$. The resulting suspension was warmed to room temperature, filtered through Celite, and eluted with ether $(150 \mathrm{~mL})$. The solution was carefully concentrated to yield 2-phenyl-4- 
pentenylamine $^{\mathrm{S} 2}(\mathbf{S 2 4}, 75 \% \mathrm{w} / \mathrm{w}$ in ether, $0.60 \mathrm{~g}, 100 \%)$. S21 was synthesized from S24 employing a procedure similar to that used to synthesize $\mathbf{S 1 5}$.

For S21: Colorless, viscous oil, 72\%. TLC (ether- $\left.\mathrm{CH}_{2} \mathrm{Cl}_{2}=1: 3\right): R_{f}=0.34 .{ }^{1} \mathrm{H}$ NMR: $\delta$ 7.177.34 (m, $6 \mathrm{H}), 5.68$ (tdd, $J=7.2,10.1,17.1 \mathrm{~Hz}, 1 \mathrm{H}), 4.94-5.03(\mathrm{~m}, 2 \mathrm{H}), 4.11(\mathrm{br} \mathrm{t}, J=5.6 \mathrm{~Hz}, 1 \mathrm{H})$, $3.65(\mathrm{ddd}, J=5.5,7.3,13.0 \mathrm{~Hz}, 1 \mathrm{H}), 3.16(\mathrm{ddd}, J=4.4,9.2,13.5 \mathrm{~Hz}, 1 \mathrm{H}), 3.09$ (dt, $J=5.6,7.2 \mathrm{~Hz}, 2$ H), 2.82-2.90 (m, $1 \mathrm{H}), 2.33-2.46(\mathrm{~m}, 2 \mathrm{H}), 1.05(\mathrm{t}, J=7.2 \mathrm{~Hz}, 3 \mathrm{H}) .{ }^{13} \mathrm{C}\left\{{ }^{1} \mathrm{H}\right\}$ NMR: $\delta \quad 158.3,142.7$, 136.2, 128.7, 128.0, 126.8, 116.6, 46.1, 45.5, 38.3, 35.3, 15.5. IR (neat, $\mathrm{cm}^{-1}$ ): 3332, 1630, 1566, 1256, 913, 698. Anal. calcd (found) for $\mathrm{C}_{14} \mathrm{H}_{20} \mathrm{~N}_{2} \mathrm{O}: \mathrm{H}, 8.68$ (8.76); C, 72.38 (72.21). HRMS calcd (found) for $\mathrm{C}_{14} \mathrm{H}_{20} \mathrm{~N}_{2} \mathrm{O}\left(\mathrm{M}^{+}\right): 232.1576(232.1581)$.

For S22: ${ }^{1} \mathrm{H}$ NMR: $\delta 11.87$ (br s, $\left.1 \mathrm{H}\right), 7.26-7.37$ (m, $\left.5 \mathrm{H}\right), 5.74(\operatorname{tdd}, J=6.8,10.3,17.1 \mathrm{~Hz}, 1$ $\mathrm{H}), 5.02-5.12(\mathrm{~m}, 2 \mathrm{H}), 3.66(\mathrm{t}, J=7.5 \mathrm{~Hz}, 1 \mathrm{H}), 2.81-2.88(\mathrm{~m}, 1 \mathrm{H}), 2.51-2.58(\mathrm{~m}, 1 \mathrm{H}) .{ }^{13} \mathrm{C}\left\{{ }^{1} \mathrm{H}\right\}$ NMR: $\delta 179.9,137.9,135.0,128.9,128.2,127.7,117.4,51.5,37.2$.

For S23: TLC (EtOAc- $\left.\mathrm{CH}_{2} \mathrm{Cl}_{2}=1: 4\right): R_{f}=0.32 .{ }^{1} \mathrm{H}$ NMR: $\delta 7.24-7.35(\mathrm{~m}, 5 \mathrm{H}), 6.02(\mathrm{br} \mathrm{s}, 1$ H), $5.71(\mathrm{tdd}, J=7.0,9.7,16.7,1 \mathrm{H}), 5.53(\mathrm{br} \mathrm{s}, 1 \mathrm{H}), 4.96-5.08(\mathrm{~m}, 2 \mathrm{H}), 3.46(\mathrm{t}, J=7.5 \mathrm{~Hz}, 1 \mathrm{H})$, 2.84-2.92 (m, $1 \mathrm{H}), 2.48-2.55(\mathrm{~m}, 1 \mathrm{H}) .{ }^{13} \mathrm{C}\left\{{ }^{1} \mathrm{H}\right\}$ NMR: $\delta \quad 175.7,139.4,135.8,128.9,128.1,127.5$, $116.9,52.6,37.2$.

For S24: ${ }^{1} \mathrm{H}$ NMR: $\delta$ 7.17-7.34 (m, $\left.5 \mathrm{H}\right), 5.68(\mathrm{tdd}, J=6.5,10.1,17.1 \mathrm{~Hz}, 1 \mathrm{H}), 4.92-5.01(\mathrm{~m}$, $2 \mathrm{H}), 2.96(\mathrm{dd}, J=4.6,12.1 \mathrm{~Hz}, 1 \mathrm{H}), 2.84(\mathrm{dd}, J=8.7,12.1 \mathrm{~Hz}, 1 \mathrm{H}), 2.64-2.71(\mathrm{~m}, 1 \mathrm{H}), 2.31-2.44$ (m, 2 H), 1.47 (br s, $2 \mathrm{H}) .{ }^{13} \mathrm{C}\left\{{ }^{1} \mathrm{H}\right\}$ NMR: $\delta$ 143.1, 136.7, 128.7, 128.1, 126.7, 116.3, 49.5, 47.5, 38.5.

$N$-Phenyl- $N^{\prime}$-(2-phenyl-4-pentenyl)urea (S25). S25 was synthesized from S24 employing a procedure similar to that used to synthesize $\mathbf{S 1 5}$. White solid, $82 \%$. TLC (ether- $\mathrm{CH}_{2} \mathrm{Cl}_{2}=1: 19$ ): $R_{f}=$ 0.37. ${ }^{1} \mathrm{H}$ NMR: $\delta 7.37$ (br s, $\left.1 \mathrm{H}\right), 7.00-7.28(\mathrm{~m}, 10 \mathrm{H}), 5.61(\operatorname{tdd}, J=7.0,10.1,17.1 \mathrm{~Hz}, 1 \mathrm{H}), 5.47$ (br t, $J=5.3 \mathrm{~Hz}, 1 \mathrm{H}), 4.90-4.97(\mathrm{~m}, 2 \mathrm{H}), 3.53(\mathrm{td}, J=6.5,13.3 \mathrm{~Hz}, 1 \mathrm{H}), 3.20(\mathrm{ddd}, J=5.0,8.7,13.5 \mathrm{~Hz}$, 
$1 \mathrm{H}), 2.76-2.83(\mathrm{~m}, 1 \mathrm{H}), 2.27-2.42(\mathrm{~m}, 2 \mathrm{H}) .{ }^{13} \mathrm{C}\left\{{ }^{1} \mathrm{H}\right\}$ NMR: $\delta \quad 156.4,142.4,139.0,136.0,129.1$, 128.7, 127.9, 126.8, 123.3, 120.5, 116.7, 45.9, 45.3, 38.1. IR (neat, $\mathrm{cm}^{-1}$ ): 3322, 1643, 1547, 1496, 1235, 694. Anal. calcd (found) for $\mathrm{C}_{18} \mathrm{H}_{20} \mathrm{~N}_{2} \mathrm{O}: \mathrm{H}, 7.19$ (7.32); C, 77.11 (77.00). HRMS calcd (found) for $\mathrm{C}_{18} \mathrm{H}_{20} \mathrm{~N}_{2} \mathrm{O}\left(\mathrm{M}^{+}\right): 280.1576(280.1574)$.

\section{Heterocyclic Products}

Phenyl 2-methyl-4,4-diphenyl-pyrrolidine-1-carboxlamide (3c). ${ }^{\mathrm{S} 4}$ Dioxane $(0.50 \mathrm{~mL})$ was added to a mixture of $2 \mathbf{c}(90 \mathrm{mg}, 0.25 \mathrm{mmol}), \mathrm{Au}(4) \mathrm{Cl}(7.5 \mathrm{mg}, 0.012 \mathrm{mmol})$, and AgOTf $(3.4 \mathrm{mg}$, $0.013 \mathrm{mmol}$ ) and the resulting suspension was stirred at room temperature for $18 \mathrm{~h}$. The resulting mixture was concentrated and chromatographed (ether- $\left.\mathrm{CH}_{2} \mathrm{Cl}_{2}=1: 30\right)$ to give $\mathbf{5}(86 \mathrm{mg}, 96 \%)$ as white microcrystals. mp 184.5-186 ${ }^{\circ} \mathrm{C}$. TLC (ether- $\left.\mathrm{CH}_{2} \mathrm{Cl}_{2}=1: 30\right): R_{f}=0.41 .{ }^{1} \mathrm{H}$ NMR: $\delta 7.44(\mathrm{~d}, J=7.7$ Hz, 2 H), 7,13-7.30 (m, 12 H), 7.01 (tt, $J=1.2,7.3 \mathrm{~Hz}, 1 \mathrm{H}), 6.54$ (br s, $1 \mathrm{H}), 4.60$ (d, $J=10.6 \mathrm{~Hz}, 1 \mathrm{H})$, 3.79-3.87 (m, $1 \mathrm{H}), 3.75$ (d, $J=10.8 \mathrm{~Hz}, 1 \mathrm{H}), 2.84(\mathrm{ddd}, J=1.4,6.3,12.3 \mathrm{~Hz}, 1 \mathrm{H}), 2.35(\mathrm{dd}, J=9.2$, $12.3 \mathrm{~Hz}, 1 \mathrm{H}), 1.34(\mathrm{~d}, 3 \mathrm{H}) .{ }^{13} \mathrm{C}\left\{{ }^{1} \mathrm{H}\right\}$ NMR: $\delta$ 154.2, 145.6, 145.2, 139.2, 128.9, 128.7, 128.5, 126.8, 126.6, 126.4, 122.9, 119.8, 56.2, 52.8, 52.1, 46.6, 20.8. IR (neat, $\mathrm{cm}^{-1}$ ): $3267,1645,1538,1443,1388$, 695. HRMS calcd (found) for $\mathrm{C}_{24} \mathrm{H}_{25} \mathrm{~N}_{2} \mathrm{O}\left(\mathrm{MH}^{+}\right): 357.1967$ (357.1969).

The remaining nitrogen heterocycles were synthesized employing a procedure analogous to that used to synthesize $\mathbf{3 c}$.

Benzyl 2-methyl-4,4-diphenylpyrrolidinecarbamate (3a). ${ }^{\mathrm{S} 3}$ Viscous colorless oil. TLC $($ EtOAc-hexanes $=1: 5): R_{f}=0.41 .{ }^{1} \mathrm{H}$ NMR $(1: 1$ ratio of rotomers): $\delta 7.11-7.40(\mathrm{~m}, 15 \mathrm{H}),[5.31(\mathrm{~d}, J$ $=12.4 \mathrm{~Hz}), 5.09(\mathrm{~d}, J=12.4 \mathrm{~Hz}), 1: 1,1 \mathrm{H}],[5.18(\mathrm{abq}, J=12.4 \mathrm{~Hz}), 1: 1,1 \mathrm{H}],[4.74(\mathrm{dd}, J=2.0,11.6$ $\mathrm{Hz}), 4.58(\mathrm{dd}, J=1.6,11.6 \mathrm{~Hz}), 1: 1,1 \mathrm{H}], 3.65-3.81(\mathrm{~m}, 2 \mathrm{H}), 2.80-2.86(\mathrm{~m}, 1 \mathrm{H}),[2.31(\mathrm{dd}, J=9.6$, $12.4 \mathrm{~Hz}), 2.26(\mathrm{dd}, J=9.6,12.8 \mathrm{~Hz}), 1: 1,1 \mathrm{H}],[1.36(\mathrm{~d}, J=6.0 \mathrm{~Hz}), 1.29(\mathrm{~d}, J=6.0 \mathrm{~Hz}), 1: 1,3 \mathrm{H}]$. 
${ }^{13} \mathrm{C}\left\{{ }^{1} \mathrm{H}\right\}$ NMR: $\delta$ [155.7, 154.9, (1:1)], [146.0, 146.0, (1:1)], [145.5, 145.3, (1:1)], [137.4, 137.3, (1:1)], $128.8,128.8,128.8,128.7,128.3,128.3,128.1,127.8,127.1,126.8,126.8,126.7,126.7,126.6,[67.1$, 66.9, (1:1)], 56.2, [53.1, 53.1, (1:1)], [52.9, 52.5, (1:1)], [47.2, 46.3, (1:1)], [21.5, 20.4, (1:1)]. IR (neat, $\mathrm{cm}^{-1}$ ): 3031, 2963, 1700, 1447, 1216, 1095. Anal. calcd (found) for $\mathrm{C}_{25} \mathrm{H}_{25} \mathrm{NO}_{2}: \mathrm{H}, 6.78$ (6.59); C, $80.83(80.83) ; \mathrm{N}, 3.77(3.80)$.

1-(2-Methyl-4,4-diphenyl-pyrrolidin-1-yl)-ethanone (3b). ${ }^{\mathrm{S} 4}$ White solid. TLC (ether- $\mathrm{CH}_{2} \mathrm{Cl}_{2}$ $=1: 2): R_{f}=0.57 .{ }^{1} \mathrm{H}$ NMR $(1: 1$ mixture of rotomers): $\delta 7.13-7.33(\mathrm{~m}, 10 \mathrm{H}),[4.99(\mathrm{dd}, J=2.4,12.1$ Hz), 4.29 (dd, $J=1.9,10.8 \mathrm{~Hz}), 1 \mathrm{H}],[4.04$ (qdd, $J=6.5,6.5,8.9 \mathrm{~Hz}), 3.77$ (qdd, $J=6.2,6.2,12.6 \mathrm{~Hz}$ ), $1 \mathrm{H}],[3.94(\mathrm{~d}, J=10.9 \mathrm{~Hz}), 3.58(\mathrm{~d}, J=12.1 \mathrm{~Hz}), 1 \mathrm{H}],[3.00$ (ddd, $J=2.6,6.8,12.6 \mathrm{~Hz}), 2.93(\mathrm{ddd}, J$ $=1.9,7.0,12.8), 1 \mathrm{H}],[2.40(\mathrm{dd}, J=8.7,12.5 \mathrm{~Hz}), 2.23(\mathrm{dd}, J=9.1,12.8 \mathrm{~Hz}), 1 \mathrm{H}],[2.11(\mathrm{~s}), 2.02(\mathrm{~s})$,

$3 \mathrm{H}],[1.33(\mathrm{~d}, J=6.2 \mathrm{~Hz}), 1.32(\mathrm{~d}, J=6.2 \mathrm{~Hz}), 3 \mathrm{H}] .{ }^{13} \mathrm{C}\left\{{ }^{1} \mathrm{H}\right\}$ NMR (1:1 mixture of rotomers): $\delta$ $169.9,168.9,145.8,145.7,145.2,145.0,128.8,128.7,128.7,128.7,126.8,126.7,126.7,126.5,126.5$, $126.4,58.1,54.6,53.1,52.8,52.5,52.3,47.4,45.7,23.5,22.1,21.6,20.2$. IR (neat, $\mathrm{cm}^{-1}$ ): 2969,2876 , 1637, 1412, 1347, 699. Anal. calcd (found) for $\mathrm{C}_{19} \mathrm{H}_{21} \mathrm{NO}$ : H, 7.58 (7.38); C, 81.68 (81.80).

Ethyl 2-methyl-4,4-diphenyl-pyrrolidine-1-carboxamide (S26). Colorless oil. TLC (ether$\left.\mathrm{CH}_{2} \mathrm{Cl}_{2}=1: 4\right): R_{f}=0.35 .{ }^{1} \mathrm{H}$ NMR: $\delta$ 7.09-7.28 $(\mathrm{m}, 10 \mathrm{H}), 4.48(\mathrm{~d}, J=10.9 \mathrm{~Hz}, 1 \mathrm{H}), 4.13(\mathrm{br} \mathrm{t}, J=$ $5.1 \mathrm{~Hz}, 1 \mathrm{H}), 3.62(\mathrm{~d}, J=10.6 \mathrm{~Hz}, 1 \mathrm{H}), 3.58-3.66(\mathrm{~m}, 1 \mathrm{H}), 3.21-3.36(\mathrm{~m}, 1 \mathrm{H}), 2.76$ (ddd, $J=1.7,6.3$, $12.1 \mathrm{~Hz}, 1 \mathrm{H}), 2.32(\mathrm{dd}, J=9.1,12.3 \mathrm{~Hz}, 1 \mathrm{H}), 1.26(\mathrm{~d}, J=6.0 \mathrm{~Hz}, 3 \mathrm{H}), 1.13$ (t, $J=7.2 \mathrm{~Hz}, 3 \mathrm{H})$. ${ }^{13} \mathrm{C}\left\{{ }^{1} \mathrm{H}\right\}$ NMR: $\delta \quad 157.0,145.9,145.6,128.6,128.6,127.0,126.6,126.6,126.4,56.1,52.9,51.8,47.1$, 35.6, 21.0, 15.9. IR (neat, $\mathrm{cm}^{-1}$ ): 3332, 2967, 1625, 1530, 1336, 698. Anal. calcd (found) for $\mathrm{C}_{20} \mathrm{H}_{24} \mathrm{~N}_{2} \mathrm{O}$ : H, 7.84 (7.79); C, 77.89 (78.04). HRMS calcd (found) for $\mathrm{C}_{20} \mathrm{H}_{24} \mathrm{~N}_{2} \mathrm{O}\left(\mathrm{M}^{+}\right)$: 308.1889, found 308.1888 . 
4-Bromophenyl 2-methyl-4,4-diphenylpyrrolidine-1-carboxamide (S27). White solid. TLC (ether- $\left.\mathrm{CH}_{2} \mathrm{Cl}_{2}=1: 19\right): R_{f}=0.69 .{ }^{1} \mathrm{H}$ NMR: $\delta 7.15-7.40(\mathrm{~m}, 14 \mathrm{H}), 6.20(\mathrm{~s}, 1 \mathrm{H}), 4.59(\mathrm{~d}, J=10.1 \mathrm{~Hz}$, $1 \mathrm{H}), 3.79-3.85(\mathrm{~d}, J=10.8 \mathrm{~Hz}, 1 \mathrm{H}), 2.89(\mathrm{dd}, J=5.1,11.3 \mathrm{~Hz}, 1 \mathrm{H}), 2.40(\mathrm{dd}, J=9.2,12.5 \mathrm{~Hz}, 1 \mathrm{H})$, $1.37(\mathrm{~d}, J=6.2 \mathrm{~Hz}, 3 \mathrm{H}) .{ }^{13} \mathrm{C}\left\{{ }^{1} \mathrm{H}\right\}$ NMR: $\delta \quad 153.8,145.5,145.1,138.3,131.9,128.8,128.7,126.9$, $126.8,126.7,126.5,121.2,115.5,56.3,52.9,52.3,46.9,20.9$. IR (neat, $\mathrm{cm}^{-1}$ ): 2972, 1643, 1518, 1401, 1238, 698. Anal. calcd (found) for $\mathrm{C}_{24} \mathrm{H}_{23} \mathrm{BrN}_{2} \mathrm{O}$ : H, 5.32 (5.20); C, 66.21 (66.02). HRMS calcd (found) for $\mathrm{C}_{24} \mathrm{H}_{23}{ }^{79} \mathrm{BrN}_{2} \mathrm{O}\left(\mathrm{M}^{+}\right)$: 434.0994 (434.0999).

4-Methoxyphenyl 2-methyl-4,4-diphenylpyrrolidine-1-carboxamide (S28). White solid. TLC (ether- $\left.\mathrm{CH}_{2} \mathrm{Cl}_{2}=1: 9\right): R_{f}=0.52 .{ }^{1} \mathrm{H}$ NMR: $\delta$ 7.14-7.33 (m, $\left.12 \mathrm{H}\right), 6.85(\mathrm{~d}, J=8.9 \mathrm{~Hz}, 2 \mathrm{H}), 6.07$ (s, $1 \mathrm{H}), 4.61(\mathrm{~d}, J=10.1 \mathrm{~Hz}, 1 \mathrm{H}), 3.77-3.83(\mathrm{~m}, 1 \mathrm{H}), 3.79(\mathrm{~s}, 3 \mathrm{H}), 3.76(\mathrm{~d}, J=10.6 \mathrm{~Hz}, 1 \mathrm{H}), 2.86$ $(\mathrm{dd}, J=6.3,12.1 \mathrm{~Hz}, 1 \mathrm{H}), 2.40(\mathrm{dd}, J=9.2,12.1 \mathrm{~Hz}, 1 \mathrm{H}), 1.37(\mathrm{~d}, J=6.2 \mathrm{~Hz}, 3 \mathrm{H}) .{ }^{13} \mathrm{C}\left\{{ }^{1} \mathrm{H}\right\} \mathrm{NMR}:$ ठ $155.9,154.6,145.7,145.3,132.2,128.8,128.7,127.0,126.8,126.6,122.0,114.3,56.3,55.7,52.9$, 52.2, 47.0, 20.9. IR (neat, $\mathrm{cm}^{-1}$ ): 3301, 1640, 1511, 1372, 1232, 699. Anal. calcd (found) for $\mathrm{C}_{25} \mathrm{H}_{26} \mathrm{~N}_{2} \mathrm{O}_{2}$ : H, 6.78 (6.56); C, 77.69 (77.42). HRMS calcd (found) for $\mathrm{C}_{25} \mathrm{H}_{26} \mathrm{~N}_{2} \mathrm{O}_{2}\left(\mathrm{M}^{+}\right)$: 386.1994 (386.1996).

4-Acetylphenyl 2-methyl-4,4-diphenylpyrrolidine-1-carboxyamide (S29). White solid. TLC (ether- $\left.\mathrm{CH}_{2} \mathrm{Cl}_{2}=1: 9\right): R_{f}=0.35 .{ }^{1} \mathrm{H} \mathrm{NMR}: \delta 7.89(\mathrm{~d}, J=8.9 \mathrm{~Hz}, 2 \mathrm{H}), 7.53(\mathrm{~d}, J=8.7 \mathrm{~Hz}, 2 \mathrm{H})$, 7.14-7.31 (m, $10 \mathrm{H}), 6.71($ br s, $1 \mathrm{H}), 4.61(\mathrm{~d}, J=9.9 \mathrm{~Hz}, 1 \mathrm{H}), 3.82-3.90(\mathrm{~m}, 1 \mathrm{H}), 3.80(\mathrm{~d}, J=10.8 \mathrm{~Hz}$ $1 \mathrm{H}), 2.89(\mathrm{dd}, J=6.5,12.1 \mathrm{~Hz}, 1 \mathrm{H}), 2.54(\mathrm{~s}, 3 \mathrm{H}), 2.39(\mathrm{dd}, J=9.2,12.3 \mathrm{~Hz}, 1 \mathrm{H}), 1.37(\mathrm{~d}, J=6.2$ $\mathrm{Hz}, 3 \mathrm{H}) .{ }^{13} \mathrm{C}\left\{{ }^{1} \mathrm{H}\right\} \mathrm{NMR}: \delta 197.2,153.4,145.4,145.0,144.0,131.6,129.9,128.8,128.7,126.8,126.7$, 126.4, 118.4, 56.3, 52.9, 52.4, 46.7, 26.5, 20.8. IR (neat, $\mathrm{cm}^{-1}$ ): 1653, 1519, 1367, 1250, 1176, 699 . HRMS calcd (found) for $\mathrm{C}_{26} \mathrm{H}_{26} \mathrm{~N}_{2} \mathrm{O}_{2}\left(\mathrm{M}^{+}\right)$: 398.1994 (398.1997). 
Phenyl 3-methyl-2-aza-spiro[4.5]decane-2-carboxamide (S30). White solid. TLC (ether$\left.\mathrm{CH}_{2} \mathrm{Cl}_{2}=1: 19\right): R_{f}=0.50 .{ }^{1} \mathrm{H}$ NMR: $\delta 7.62(\mathrm{t}, J=8.0 \mathrm{~Hz}, 2 \mathrm{H}), 7.25-7.29(\mathrm{~m}, 2 \mathrm{H}), 6.98-7.02(\mathrm{~m}, 1$ $\mathrm{H}), 6.13($ br s, $1 \mathrm{H}), 3.32(\mathrm{~s}, 2 \mathrm{H}), 1.83(\mathrm{~s}, 2 \mathrm{H}), 1.54(\mathrm{~s}, 6 \mathrm{H}), 1.38-1.59(\mathrm{~m}, 10 \mathrm{H}) .{ }^{13} \mathrm{C}\left\{{ }^{1} \mathrm{H}\right\} \mathrm{NMR}: \delta$ $153.8,139.4,128.9,122.7,119.8,61.7,58.4,54.2,40.0,37.6,28.9,26.0,23.5$. IR (neat, $\left.\mathrm{cm}^{-1}\right): 3297$, 2924, 2854, 1637, 1367, 757. Anal. calcd (found) for $\mathrm{C}_{17} \mathrm{H}_{24} \mathrm{~N}_{2} \mathrm{O}: \mathrm{H}, 8.88$ (8.82); C, 74.96 (74.92). HRMS calcd (found) for $\mathrm{C}_{17} \mathrm{H}_{24} \mathrm{~N}_{2} \mathrm{O}\left(\mathrm{M}^{+}\right)$: 286.2045 (286.2043).

Ethyl 3,3,5-trimethyl-2-phenyl-pyrrolidine-1-carboxamide (S31). Chromatography of the crude reaction product gave three fractions, one of which consisted of a >20:1 mixture of cis-S31:transS31, a second that consisted of a 14:1 mixture of cis-S31:trans-S31, and a third that consisted of a 1:2.8 mixture of cis-S31:trans-S31. Spectroscopic analysis of these fractions allowed assignment of the ${ }^{1} \mathrm{H}$ and ${ }^{13} \mathrm{C}\left\{{ }^{1} \mathrm{H}\right\}$ NMR resonances corresponding to cis-S31 and trans-S31, respectively. Combination of these fractions gave $\mathbf{S 3 1}$ [99\%, 3.9:1 (cis/trans)] as a white solid. TLC (ether- $\left.\mathrm{CH}_{2} \mathrm{Cl}_{2}=1: 5\right): R_{f}=0.44$. ${ }^{1} \mathrm{H}$ NMR (cis-S31): $\delta$ 7.23-7.37 (m, $\left.5 \mathrm{H}\right), 4.19$ (s, $\left.1 \mathrm{H}\right), 4.09-4.18$ (m, $\left.1 \mathrm{H}\right), 3.80$ (br t, $\left.J=5.5 \mathrm{~Hz}, 1 \mathrm{H}\right)$, 3.00-3.19 (m, $2 \mathrm{H}), 1.76(\mathrm{dd}, J=6.7,12.5 \mathrm{~Hz}, 1 \mathrm{H}), 1.53(\mathrm{~d}, J=6.2 \mathrm{~Hz}, 3 \mathrm{H}), 1.51(\mathrm{dd}, J=10.3,12.8$ $\mathrm{Hz}, 1 \mathrm{H}), 1.18(\mathrm{~s}, 3 \mathrm{H}), 0.92(\mathrm{t}, J=7.2 \mathrm{~Hz}, 3 \mathrm{H}), 0.57(\mathrm{~s}, 3 \mathrm{H}) .{ }^{13} \mathrm{C}\left\{{ }^{1} \mathrm{H}\right\} \mathrm{NMR}(\operatorname{cis}-\mathbf{S 3 1}): \delta \quad 158.3$, 141.2, 128.7, 127.8, 127.2, 74.0, 52.9, 45.9, 42.1, 35.2, 29.6, 25.3, 21.1, 15.5. ${ }^{1} \mathrm{H}$ NMR (trans-S31): $\delta$ 7.26-7.32 (m, $3 \mathrm{H}), 7.08(\mathrm{~d}, J=7.5 \mathrm{~Hz}, 2 \mathrm{H}), 4.29-4.37(\mathrm{~m}, 1 \mathrm{H}), 4.26(\mathrm{~s}, 1 \mathrm{H}), 3.78$ (br t, $J=5.8 \mathrm{~Hz}, 1$ H), 2.88-3.13 (m, $2 \mathrm{H}), 2.10(\mathrm{dd}, J=9.1,13.0 \mathrm{~Hz}, 1 \mathrm{H}), 1.50(\mathrm{~d}, J=6.3 \mathrm{~Hz}, 3 \mathrm{H}), 1.46(\mathrm{dd}, J=3.6$, $13.0 \mathrm{~Hz}, 1 \mathrm{H}), 1.31(\mathrm{~s}, 3 \mathrm{H}), 0.71(\mathrm{t}, J=7.2 \mathrm{~Hz}, 3 \mathrm{H}), 0.57(\mathrm{~s}, 3 \mathrm{H}) .{ }^{13} \mathrm{C}\left\{{ }^{1} \mathrm{H}\right\} \mathrm{NMR}($ trans-S31): $\delta$ 157.2, 140.8, 128.6, 127.7, 127.2, 72.8, 54.3, 45.2, 42.5, 35.2, 30.5, 25.9, 22.2, 15.3. IR (neat, $\left.\mathrm{cm}^{-1}\right)$ : 3397, 2963, 1626, 1519, 1324, 697. HRMS calcd (found) for $\mathrm{C}_{16} \mathrm{H}_{24} \mathrm{~N}_{2} \mathrm{O}\left(\mathrm{M}^{+}\right)$: 260.1889 (260.1894).

The stereochemistry of the major diastereomer of $\mathbf{S 3 1}$ (cis-S31) was determined via ${ }^{1} \mathrm{H}-{ }^{1} \mathrm{H}$ NOESY analysis (Figures S1 and S3). A cis relationship between $\mathrm{Me}_{\mathrm{a}}$ and $\mathrm{H}_{\mathrm{Ph}}$ was established by the 
much stronger cross peak between $\mathrm{Me}_{\mathrm{b}}$ and $\mathrm{H}_{\mathrm{Bn}}$ relative to the cross peak between $\mathrm{Me}_{\mathrm{a}}$ and $\mathrm{H}_{\mathrm{Bn}}$ and from the presence of a cross peak between $\mathrm{Me}_{\mathrm{a}}$ and $\mathrm{H}_{\mathrm{Ph}}$ and the absence of a detectable cross peak between $\mathrm{Me}_{\mathrm{b}}$ and $\mathrm{H}_{\mathrm{Ph}}$. The cis relationship between $\mathrm{H}_{\mathrm{b}}$ and $\mathrm{Me}_{\mathrm{b}}$ was established by the greater magnitude of the $\mathrm{Me}_{\mathrm{b}} / \mathrm{H}_{\mathrm{b}}$ cross peak relative to the $\mathrm{Me}_{\mathrm{a}} / \mathrm{H}_{\mathrm{b}}$ cross peak. The overlapping resonances corresponding to $\mathrm{H}_{\mathrm{a}}$ and $\mathrm{Me}_{\mathrm{c}}$ in the ${ }^{1} \mathrm{H}$ NMR spectrum of cis-S31 complicated, stereochemical analysis. Nevertheless, the cis relationship between $\mathrm{H}_{\mathrm{a}}$ and $\mathrm{Me}_{\mathrm{c}}$ was established from the NOESY spectrum. First, was the presence of a cross peak between $\mathrm{H}_{\mathrm{a}} / \mathrm{Me}_{\mathrm{c}}$ and $\mathrm{Me}_{\mathrm{a}}$ and the absence of a cross peak between $\mathrm{H}_{\mathrm{a}} / \mathrm{Me}_{\mathrm{c}}$ and $\mathrm{Me}_{\mathrm{b}}$. Second was the presence of a cross peak between $\mathrm{H}_{\mathrm{Ph}}$ and $\mathrm{H}_{\mathrm{a}} / \mathrm{Me}_{\mathrm{c}}$ and the absence of cross peaks between either $\mathrm{H}_{\mathrm{Ph}}$ and $\mathrm{H}_{\mathrm{c}}$ or between $\mathrm{H}_{\mathrm{Ph}}$ and $\mathrm{H}_{\mathrm{b}}$. Therefore $\mathrm{H}_{\mathrm{a}}$ and $\mathrm{Me}_{\mathrm{c}}$ must be cis to one another and, because $\mathrm{H}_{\mathrm{b}}$ is trans to $\mathrm{H}_{\mathrm{Ph}}, \mathrm{Me}_{\mathrm{c}}$ must be cis to $\mathrm{H}_{\mathrm{Ph}}$. Taken together, these data establish the cis relationship between $\mathrm{Me}_{\mathrm{c}}$ and $\mathrm{H}_{\mathrm{Ph}}$.

Figure S1. Relevant cross-peaks and NOE enhancements observed in the ${ }^{1} \mathrm{H}-{ }^{1} \mathrm{H}$ NOESY spectrum of cis-S31 (all values reported are absolute enhancements and not standardized).

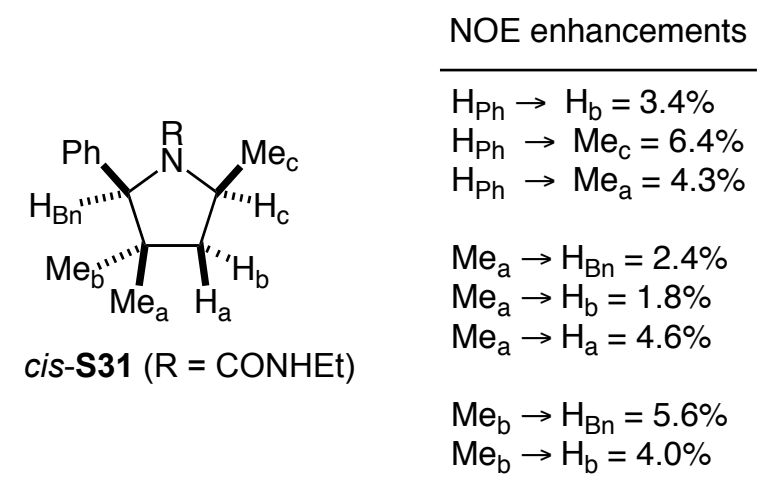

Phenyl 3,3,5-trimethyl-2-phenyl-pyrrolidine-1-carboxamide (S32). Chromatography of the crude reaction product gave two fractions, one of which consisted of a $>20: 1$ mixture of cis-S32:trans- 
S32 and a second that consisted of 3.4:1 mixture of cis-S32:trans-S32. Spectroscopic analysis of these fractions allowed assignment of the ${ }^{1} \mathrm{H}$ and ${ }^{13} \mathrm{C}\left\{{ }^{1} \mathrm{H}\right\}$ NMR resonances corresponding to cis-S32 and trans-S32. Combination of these fractions gave a 3.6:1 mixture of cis-S32:trans-S32 (92\%) as a white solid. TLC (ether- $\left.\mathrm{CH}_{2} \mathrm{Cl}_{2}=1: 19\right): R_{f}=0.54 .{ }^{1} \mathrm{H}$ NMR (cis-S32): $\delta \quad 7.32-7.45(\mathrm{~m}, 5 \mathrm{H}), 7.15-7.19(\mathrm{~m}$, $2 \mathrm{H}), 7.07-7.09$ (m, $2 \mathrm{H}), 6.92(\mathrm{td}, J=1.2,7.2 \mathrm{~Hz}, 1 \mathrm{H}), 5.92(\mathrm{~s}, 1 \mathrm{H}), 4.37(\mathrm{~s}, 1 \mathrm{H}), 4.21-4.29(\mathrm{~m}, 1 \mathrm{H})$, $1.85(\mathrm{dd}, J=6.5,12.6 \mathrm{~Hz}, 1 \mathrm{H}), 1.61(\mathrm{dd}, J=10.1,12.6 \mathrm{~Hz}, 1 \mathrm{H}), 1.58(\mathrm{~d}, J=6.0 \mathrm{~Hz}, 3 \mathrm{H}), 1.25(\mathrm{~s}, 3$ H), $0.64(\mathrm{~s}, 3 \mathrm{H}) .{ }^{13} \mathrm{C}\left\{{ }^{1} \mathrm{H}\right\}$ NMR (cis-S32): $\delta$ 155.3, 140.4, 139.1, 129.1, 128.8, 128.3, 127.4, 122.6, 119.1, 74.3, 53.1, 45.8, 42.2, 29.6, 25.2, 20.6. ${ }^{1} \mathrm{H}$ NMR (trans-S32): $\delta \quad 6.89-7.46$ (m, $\left.10 \mathrm{H}\right), 5.94$ (s, 1 H), $4.46(\mathrm{~s}, 1 \mathrm{H}), 4.40-4.44(\mathrm{~m}, 1 \mathrm{H}), 2.17(\mathrm{dd}, J=9.1,13.0 \mathrm{~Hz}, 1 \mathrm{H}), 1.59(\mathrm{~d}, J=6.3 \mathrm{~Hz}, 3 \mathrm{H}), 1.54$ $(\mathrm{dd}, J=3.6,13.0 \mathrm{~Hz}, 1 \mathrm{H}), 1.36(\mathrm{~s}, 3 \mathrm{H}), 0.63(\mathrm{~s}, 3 \mathrm{H}) .{ }^{13} \mathrm{C}\left\{{ }^{1} \mathrm{H}\right\} \mathrm{NMR}($ trans-S32): $\delta \quad 154.2,140.0$, 139.0, 128.8, 128.7, 128.1, 127.4, 122.7, 119.5, 73.0, 54.5, 45.2, 42.6, 30.3, 25.8, 22.0. IR (neat, $\mathrm{cm}^{-1}$ ): 3409, 2961, 1664, 1524, 1440, 1331. HRMS calcd (found) for $\mathrm{C}_{20} \mathrm{H}_{24} \mathrm{~N}_{2} \mathrm{O}\left(\mathrm{M}^{+}\right): 308.1889$ (308.1892).

Phenyl 2-methyl-4-phenyl-pyrrolidine-1-carboxamide (S33). Chromatography of the crude reaction product gave two fractions, one of which consisted of a >20:1 mixture of cis-S33:trans-S33 and a second that consisted of 3.7:1 mixture of cis-S33:trans-S33. Spectroscopic analysis of these fractions allowed assignment of the ${ }^{1} \mathrm{H}$ and ${ }^{13} \mathrm{C}\left\{{ }^{1} \mathrm{H}\right\}$ NMR resonances corresponding to cis-S33 and trans-S33. Combination of these fractions gave $\mathbf{S 3 3}$ [99\%, 2.9:1 (cis/trans)] as a white solid. TLC (ether- $\mathrm{CH}_{2} \mathrm{Cl}_{2}=$ 1:19): $R_{f}=0.19 .{ }^{1} \mathrm{H}$ NMR (cis-S33): $\delta$ 7.22-7.40 (m, $\left.9 \mathrm{H}\right), 6.96-7.01(\mathrm{~m}, 1 \mathrm{H}), 6.23(\mathrm{br} \mathrm{s}, 1 \mathrm{H}), 4.06-$ $4.14(\mathrm{~m}, 1 \mathrm{H}), 3.96(\mathrm{dd}, J=8.0,8.2 \mathrm{~Hz}, 1 \mathrm{H}), 3.25-3.42(\mathrm{~m}, 2 \mathrm{H}), 2.52-2.58(\mathrm{~m}, 1 \mathrm{H}), 1.77$ (dt, $J=9.4$, $9.6 \mathrm{~Hz}, 1 \mathrm{H}), 1.41(\mathrm{~d}, J=6.0 \mathrm{~Hz}, 3 \mathrm{H}) .{ }^{13} \mathrm{C}\left\{{ }^{1} \mathrm{H}\right\} \mathrm{NMR}($ cis-S33): $\delta \quad 154.2,140.3,139.3,129.0,128.8$, 127.3, 127.1, 122.9, 119.6, 54.0, 53.5, 43.4, 42.1, 21.3. ${ }^{1} \mathrm{H}$ NMR (trans-S33): $\delta$ 7.22-7.40 (m, $\left.9 \mathrm{H}\right)$, 6.96-7.01 (m, $1 \mathrm{H}), 6.22(\mathrm{br} \mathrm{s}, 1 \mathrm{H}), 4.27-4.33$ (m, $1 \mathrm{H}), 3.87$ (dd, $J=8.4,8.5 \mathrm{~Hz}, 1 \mathrm{H}), 3.59-3.68(\mathrm{~m}, 1$ H), 3.34-3.40 (m, $1 \mathrm{H}), 2.23(\mathrm{dt}, J=8.0,12.1 \mathrm{~Hz}, 1 \mathrm{H}), 1.98(\mathrm{dd}, J=6.5,12.3 \mathrm{~Hz}, 1 \mathrm{H}), 1.34(\mathrm{~d}, J=6.5$ 
$\mathrm{Hz}, 3 \mathrm{H}) .{ }^{13} \mathrm{C}\left\{{ }^{1} \mathrm{H}\right\}$ NMR (trans-S33): $\delta \quad 153.7,140.8,139.2,129.0,128.9,127.2,127.2,122.9,119.6$, 53.5, 53.0, 42.2, 40.0, 21.1. IR (neat, $\mathrm{cm}^{-1}$ ): 3276, 1639, 1532, 1443, 1377, 756. HRMS calcd (found) for $\mathrm{C}_{18} \mathrm{H}_{20} \mathrm{~N}_{2} \mathrm{O}\left(\mathrm{M}^{+}\right): 280.1576(280.1574)$.

Ethyl 2-methyl-4-phenyl-pyrrolidine-1-carboxamide (S34). Chromatography of the crude reaction product gave two fractions, one of which consisted of a >20:1 mixture of cis-S34:trans-S34 and a second that consisted of 3.0:1 mixture of cis-S34:trans-S34. Spectroscopic analysis of these fractions allowed assignment of the ${ }^{1} \mathrm{H}$ and ${ }^{13} \mathrm{C}\left\{{ }^{1} \mathrm{H}\right\}$ NMR resonances corresponding to cis-S34 and trans-S34. Combination of these fractions gave a 3.3:1 mixture of cis-S34:trans-S34 S34 (84\%) as a white solid. TLC (ether- $\left.\mathrm{CH}_{2} \mathrm{Cl}_{2}=1: 19\right): R_{f}=0.21 .{ }^{1} \mathrm{H}$ NMR (cis-S34): $\delta$ 7.22-7.34 (m, $\left.5 \mathrm{H}\right), 4.13(\mathrm{br} \mathrm{t}, J=5.3$ Hz, $1 \mathrm{H}), 3.86-4.03$ (m, $2 \mathrm{H}), 3.21-3.67(\mathrm{~m}, 4 \mathrm{H}), 2.50-2.56(\mathrm{~m}, 1 \mathrm{H}), 1.69-1.78(\mathrm{~m}, 1 \mathrm{H}), 1.37(\mathrm{~d}, J=$ $6.2 \mathrm{~Hz}, 3 \mathrm{H}), 1.15(\mathrm{t}, J=7.3 \mathrm{~Hz}, 3 \mathrm{H}) .{ }^{13} \mathrm{C}\left\{{ }^{1} \mathrm{H}\right\}$ NMR (cis-S34): $\delta \quad 157.1,140.8,128.7,127.2,126.9$, 53.5, 53.1, 43.2, 42.3, 35.5, 21.5, 15.8. ${ }^{1} \mathrm{H}$ NMR (trans-S34): $\delta$ 7.21-7.33 (m, $\left.5 \mathrm{H}\right), 4.18$ (br t, $J=6.2$ $\mathrm{Hz}, 1 \mathrm{H}), 3.73(\mathrm{dd}, J=8.4,8.5 \mathrm{~Hz}, 1 \mathrm{H}), 3.54-3.62(\mathrm{~m}, 1 \mathrm{H}), 3.20-3.36(\mathrm{~m}, 4 \mathrm{H}), 2.13-2.21(\mathrm{~m}, 1 \mathrm{H})$, $1.94(\mathrm{dd}, J=6.2,12.1 \mathrm{~Hz}, 1 \mathrm{H}), 1.27(\mathrm{~d}, J=6.3 \mathrm{~Hz}, 3 \mathrm{H}), 1.13(\mathrm{t}, J=7.2 \mathrm{~Hz}, 3 \mathrm{H}) .{ }^{13} \mathrm{C}\left\{{ }^{1} \mathrm{H}\right\} \mathrm{NMR}$ (trans-S34): $\delta 156.6,141.2,128.7,127.2,127.0,53.1,52.7,42.1,40.1,35.4,21.2,15.9 . \quad$ IR (neat, $\mathrm{cm}^{-}$ ${ }^{1}$ ): $3335,2968,1626,1532,1369,761$. HRMS calcd (found) for $\mathrm{C}_{14} \mathrm{H}_{20} \mathrm{~N}_{2} \mathrm{O}\left(\mathrm{M}^{+}\right): 232.1576$ (232.1569).

The cis-stereochemistry of the major diastereomer of $\mathbf{S 3 4}$ was established by combined ${ }^{1} \mathrm{H}-{ }^{1} \mathrm{H}$ NOESY and ${ }^{1} \mathrm{H}-{ }^{1} \mathrm{H}$ COSY analysis of the major diastereomer of $\mathbf{S 3 4}$ (Figures S2, S4, \& S5). $1 \mathrm{H}$ assignments were made on the basis of ${ }^{1} \mathrm{H}-{ }^{1} \mathrm{H}$ COSY analysis. The cis relationship between the menthyl group and phenyl group of the pyrrolidine ring was established from the presence of a cross peak between the aryl protons and the methyl group. Furthermore, the cis relationship between $\mathrm{H}_{\mathrm{Ph}}$ and $\mathrm{H}_{\mathrm{b}}$ was established by the presence of a cross peak between $\mathrm{H}_{\mathrm{Ph}}$ and $\mathrm{H}_{\mathrm{b}}$ and the absence of a cross peak 
betweeh $\mathrm{H}_{\mathrm{Ph}}$ and $\mathrm{H}_{\mathrm{c}}$. The cis relationship between $\mathrm{H}_{\mathrm{b}}$ and Me was established by the presence of a cross peak between Me and $\mathrm{H}_{\mathrm{b}}$ and the absence of a cross peak between Me and $\mathrm{H}_{\mathrm{c}}$.

Figure S2. Proton assignments for cis-S34.

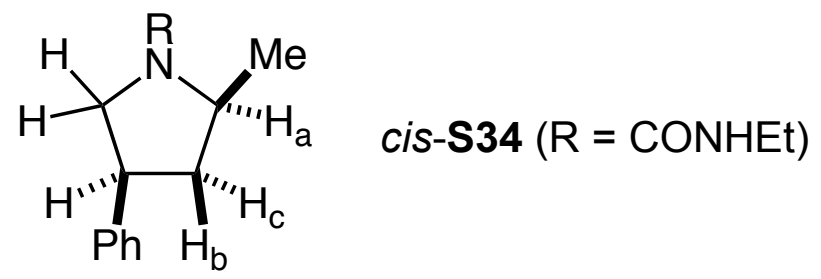

Phenyl 4-isopropyl-2-methyl-pyrrolidine-1-carboxamide (S35). Chromatography of the crude reaction product gave two fractions, one of which consisted of a 6.3:1 mixture of cis-S35:transS35, and a second that consisted of 4.1:1 mixture of cis-S35:trans-S35. Spectroscopic analysis of these fractions allowed assignment of the ${ }^{1} \mathrm{H}$ and ${ }^{13} \mathrm{C}\left\{{ }^{1} \mathrm{H}\right\}$ NMR resonances corresponding to cis-S35 and partial assignment of the ${ }^{1} \mathrm{H}$ and ${ }^{13} \mathrm{C}\left\{{ }^{1} \mathrm{H}\right\}$ NMR resonances corresponding to trans-S35. Combination of these fractions gave a 5.5:1 mixture of cis-S35:trans-S35. S35 (98\%) as a white solid. TLC (ether$\left.\mathrm{CH}_{2} \mathrm{Cl}_{2}=1: 11\right): R_{f}=0.62 .{ }^{1} \mathrm{H}$ NMR (cis-S35): $\delta$ 7.37-7.42 (m, $\left.2 \mathrm{H}\right)$, 7.25-7.29 (m, $\left.2 \mathrm{H}\right)$, 6.98-7.02 (m, $1 \mathrm{H}), 6.11(\mathrm{br} \mathrm{s}, 1 \mathrm{H}), 3.92-4.01(\mathrm{~m}, 1 \mathrm{H}), 3.72(\mathrm{t}, J=8.4 \mathrm{~Hz}, 1 \mathrm{H}), 2.99-3.04(\mathrm{~m}, 1 \mathrm{H}), 2.32(\mathrm{td}, J=6.7$, $12.5 \mathrm{~Hz}, 1 \mathrm{H}), 1.73-1.85(\mathrm{~m}, 1 \mathrm{H}), 1.44-1.53(\mathrm{~m}, 1 \mathrm{H}), 1.34(\mathrm{~d}, J=6.0 \mathrm{~Hz}, 3 \mathrm{H}), 1.20-1.28(\mathrm{~m}, 2 \mathrm{H})$, 0.92-0.96 (m, $6 \mathrm{H}) .{ }^{13} \mathrm{C}\left\{{ }^{1} \mathrm{H}\right\}$ NMR (cis-S35): $\delta \quad 154.3,139.4,128.9,122.7,119.5,54.0,51.6,45.7$, 40.0, 32.1, 21.6, 21.4, 21.2. ${ }^{1} \mathrm{H}$ NMR (trans-S35, partial): $\delta 6.14(\mathrm{br} \mathrm{s}, 1 \mathrm{H}), 4.14-4.21(\mathrm{~m}, 1 \mathrm{H}), 3.61$ $(\mathrm{dd}, J=8.0,8.7 \mathrm{~Hz}, 1 \mathrm{H}), 3.00-3.05(\mathrm{~m}, 1 \mathrm{H}), 2.07-2.19(\mathrm{~m}, 1 \mathrm{H}), 1.63-1.72(\mathrm{~m}, 1 \mathrm{H}), 1.34(\mathrm{~d}, J=6.0$ $\mathrm{Hz}, 3 \mathrm{H}) .{ }^{13} \mathrm{C}\left\{{ }^{1} \mathrm{H}\right\}$ NMR (trans-S35): $\delta \quad 154.3,139.4,128.9,122.7,119.6,53.5,50.7,44.4,37.4,32.5$, 21.3, 21.2, 21.0. IR (neat, $\mathrm{cm}^{-1}$ ): 3223, 2959, 1633, 1443, 1385, 756. Anal. calcd (found) for 
$\mathrm{C}_{15} \mathrm{H}_{22} \mathrm{~N}_{2} \mathrm{O}$ : H, 9.00 (9.00); C, 73.13 (72.90). HRMS calcd (found) for $\mathrm{C}_{15} \mathrm{H}_{22} \mathrm{~N}_{2} \mathrm{O}\left(\mathrm{M}^{+}\right.$): 246.1732 (246.1731).

Benzyl 2-methyl-pyrrolidine-1-carboxamide (S36). TLC (ether- $\mathrm{CH}_{2} \mathrm{Cl}_{2}=1: 2$ ): $\quad R_{f}=0.46$. White solid. ${ }^{1} \mathrm{H}$ NMR: $\delta$ 7.22-7.33 (m, $\left.5 \mathrm{H}\right), 4.53$ (br t, $\left.1 \mathrm{H}\right), 4.36-4.48(\mathrm{~m}, 2 \mathrm{H}), 3.96-4.03(\mathrm{~m}, 1 \mathrm{H})$, 3.21-3.34 (m, $2 \mathrm{H}), 1.82-2.03(\mathrm{~m}, 3 \mathrm{H}), 1.54-1.61(\mathrm{~m}, 1 \mathrm{H}), 1.18(\mathrm{~d}, J=6.3 \mathrm{~Hz}, 3 \mathrm{H}) .{ }^{13} \mathrm{C}\left\{{ }^{1} \mathrm{H}\right\} \mathrm{NMR}: \delta$ 156.6, 140.0, 128.6, 127.8, 127.2, 52.8, 45.9, 44.7, 32.9, 23.6, 20.7. IR (neat, $\mathrm{cm}^{-1}$ ): 3334, 2962, 1626, 1533, 1389, 1350. HRMS calcd (found) for $\mathrm{C}_{13} \mathrm{H}_{18} \mathrm{~N}_{2} \mathrm{O}\left(\mathrm{M}^{+}\right)$: 218.1419 (218.1418).

Ethyl 3-methyl-2-aza-spiro[5.5] undecane-2-carboxamide (S37). TLC (ether- $\mathrm{CH}_{2} \mathrm{Cl}_{2}=1: 3$ ): $R_{f}=0.50$. Colorless oil. ${ }^{1} \mathrm{H}$ NMR: $\delta 4.41(\mathrm{br} \mathrm{t}, J=5.3 \mathrm{~Hz}, 1 \mathrm{H}), 4.08-4.16(\mathrm{~m}, 1 \mathrm{H}), 3.64(\mathrm{~d}, J=13.5$ Hz, $1 \mathrm{H}), 3.13-3.29$ (m, $2 \mathrm{H}), 2.48$ (d, $J=13.5 \mathrm{~Hz}, 1 \mathrm{H}), 1.76-1.86(\mathrm{~m}, 1 \mathrm{H}), 1.17-1.43$ (m, $13 \mathrm{H}), 1.08$ (t, $J=6.7 \mathrm{~Hz}, 3 \mathrm{H}), 1.07(\mathrm{~d}, J=7.0 \mathrm{~Hz}, 3 \mathrm{H}) .{ }^{13} \mathrm{C}\left\{{ }^{1} \mathrm{H}\right\} \mathrm{NMR}: \delta \quad 157.8,47.2,46.2,38.1,35.7,33.1$, 31.4, 30.2, 26.7, 25.7, 21.8, 21.5, 15.7, 15.6. IR (neat, $\mathrm{cm}^{-1}$ ): 3344, 2925, 2855, 1617, 1532, 1295. HRMS calcd (found) for $\mathrm{C}_{14} \mathrm{H}_{26} \mathrm{~N}_{2} \mathrm{O}\left(\mathrm{M}^{+}\right)$: 238.2045 (238.2043). 
Figure S3. ${ }^{1} \mathrm{H}-{ }^{1} \mathrm{H}$ NOESY of $c i s-\mathbf{S 3 1}$.

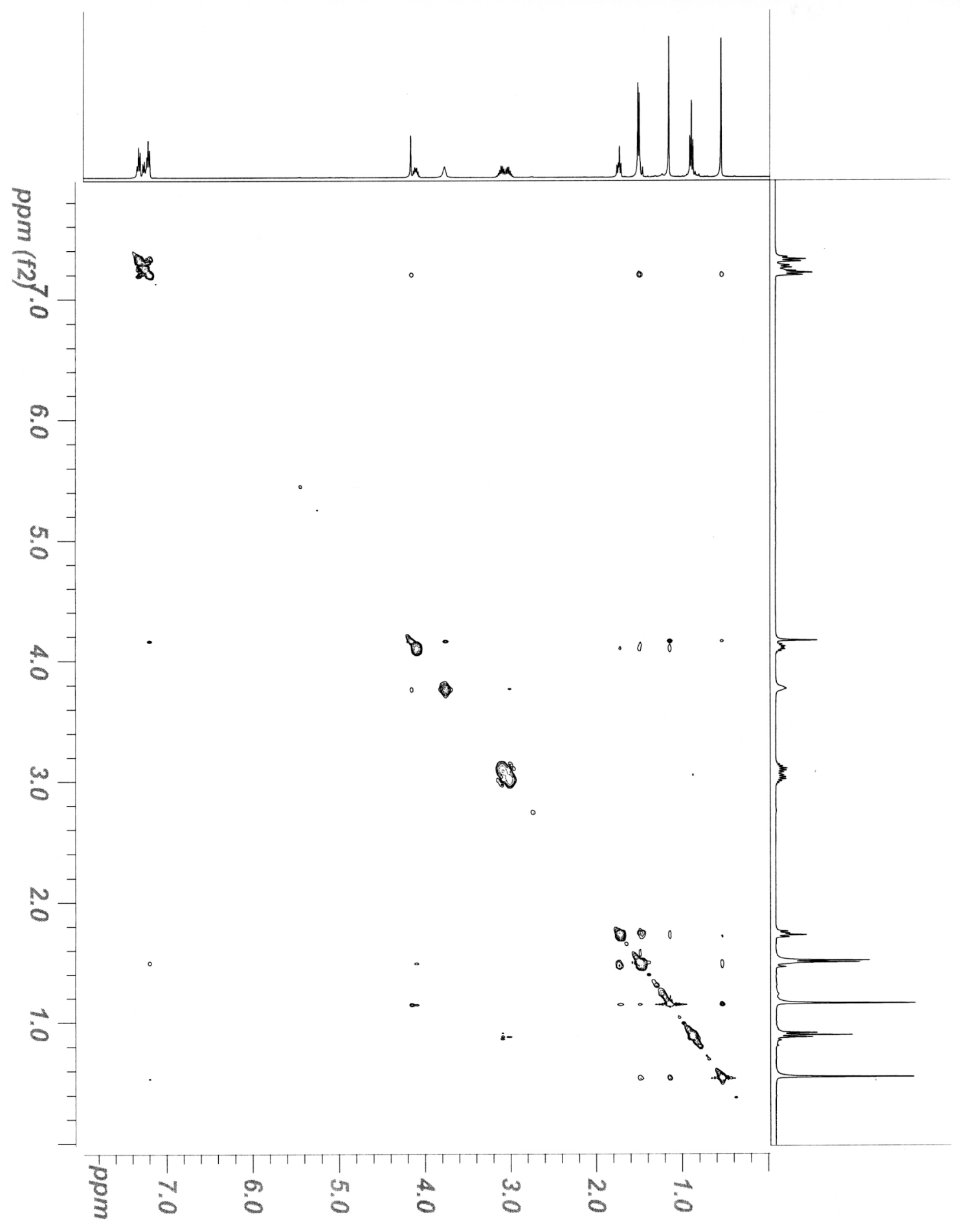


Figure S4. Partial ${ }^{1} \mathrm{H}-{ }^{1} \mathrm{H}$ COSY of $c i s-S 34$.

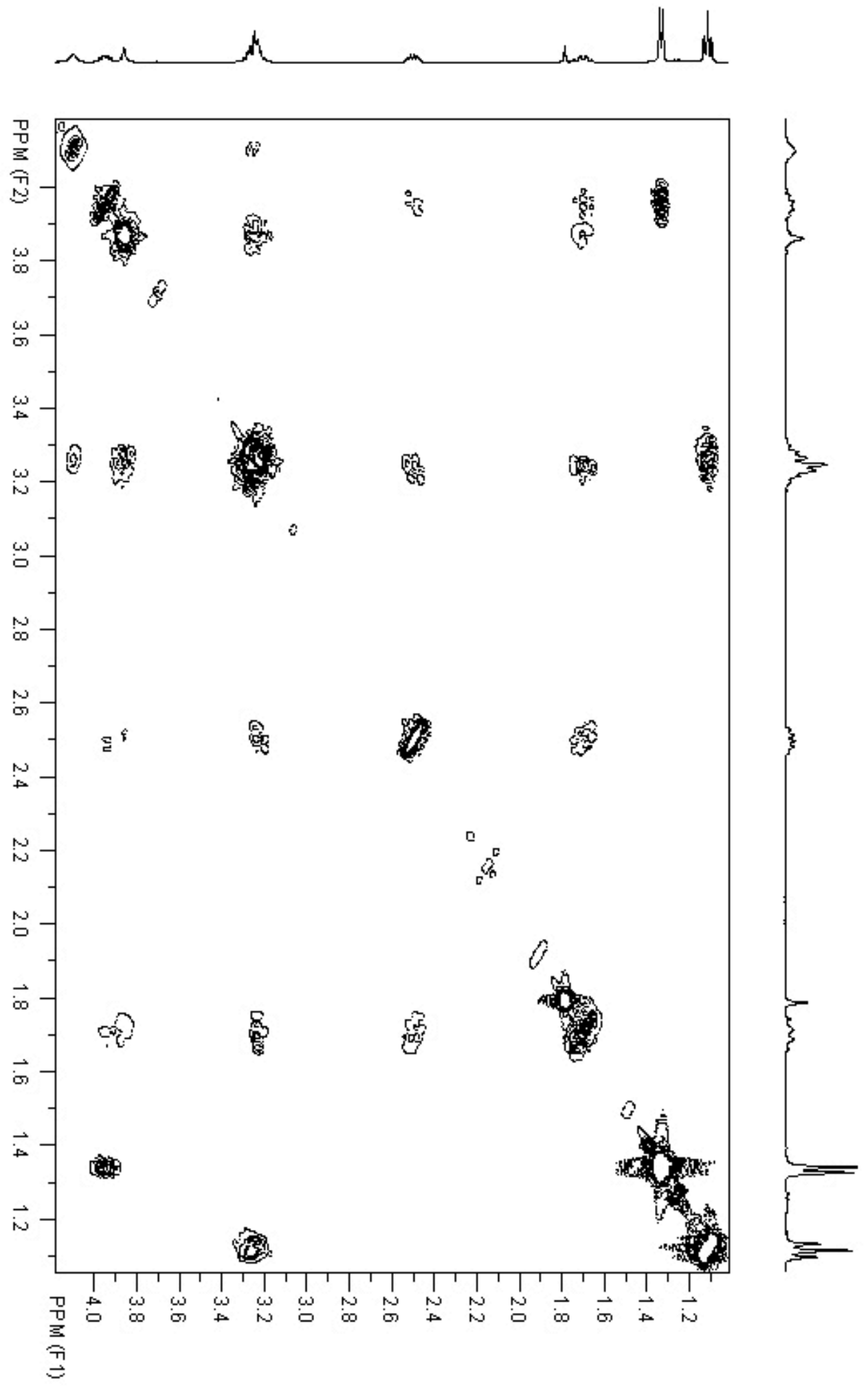


Figure S5. Partial ${ }^{1} \mathrm{H}-{ }^{1} \mathrm{H}$ NOESY of $c i s-$ S34.

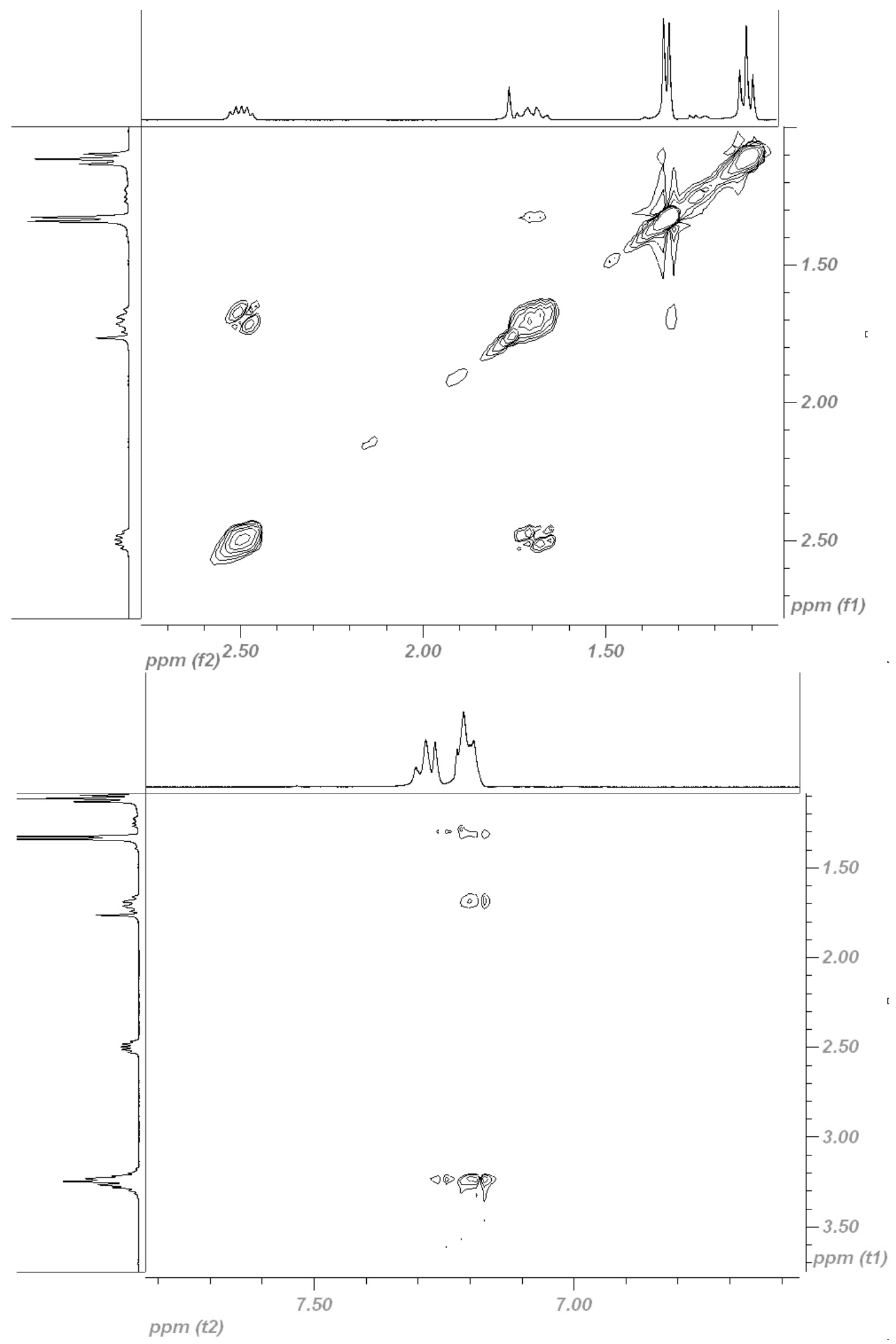


Figure S6. ${ }^{1} \mathrm{H}$ NMR spectrum of $\mathbf{2 c}$.

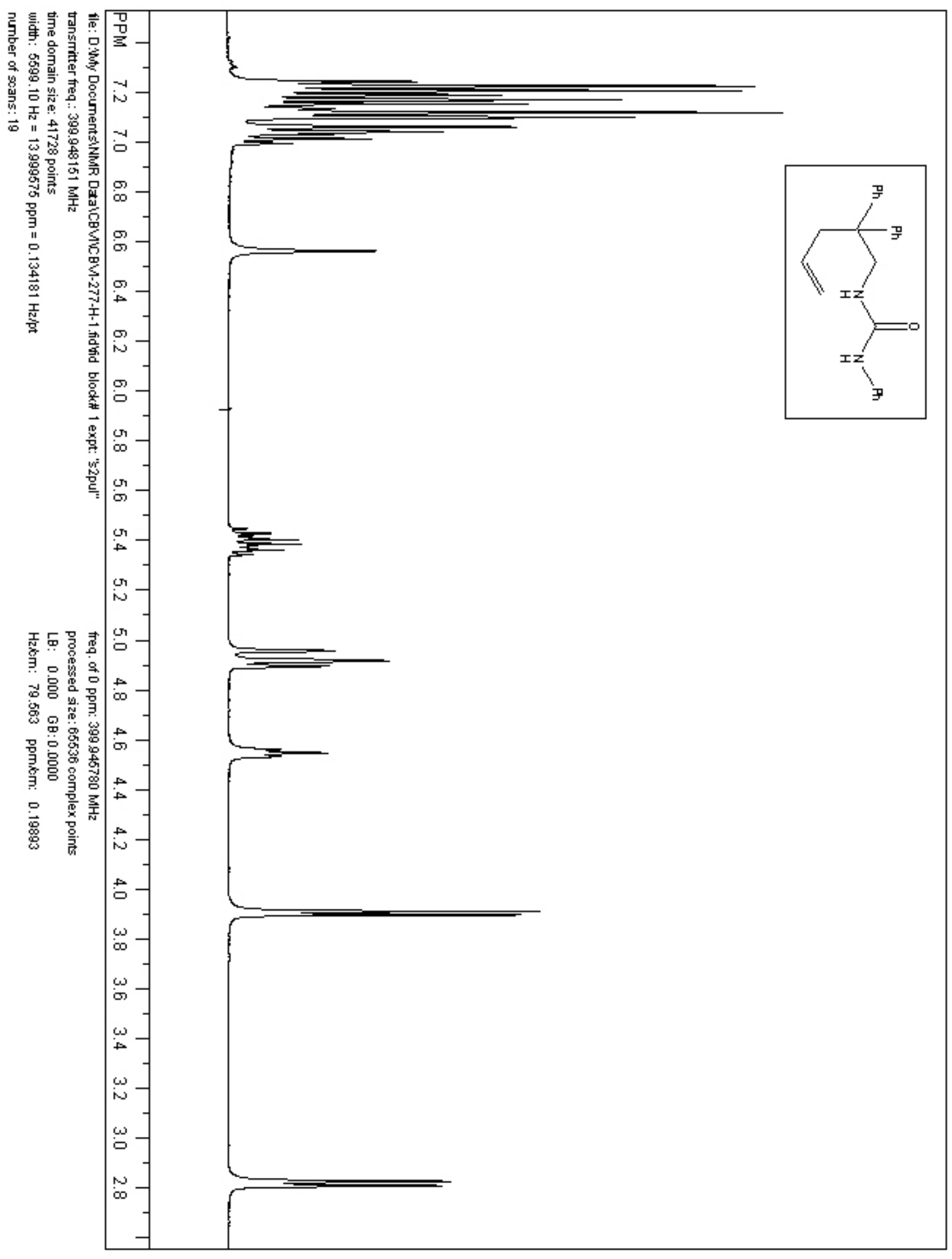


Figure S7. ${ }^{13} \mathrm{C}\left\{{ }^{1} \mathrm{H}\right\}$ NMR spectrum of $\mathbf{2 c}$.

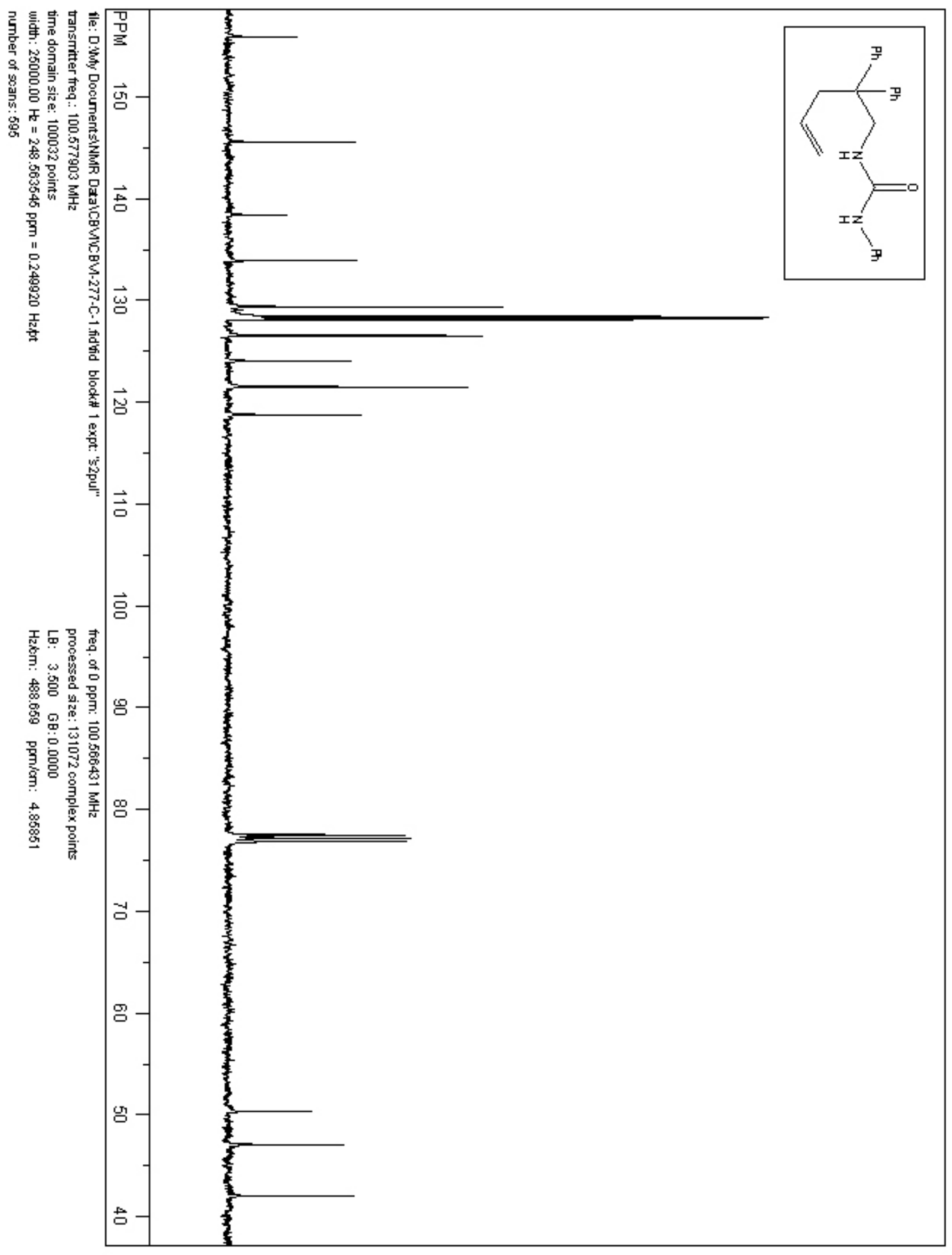


Figure S8. ${ }^{1} \mathrm{H}$ NMR spectrum of $\mathbf{3 c}$.

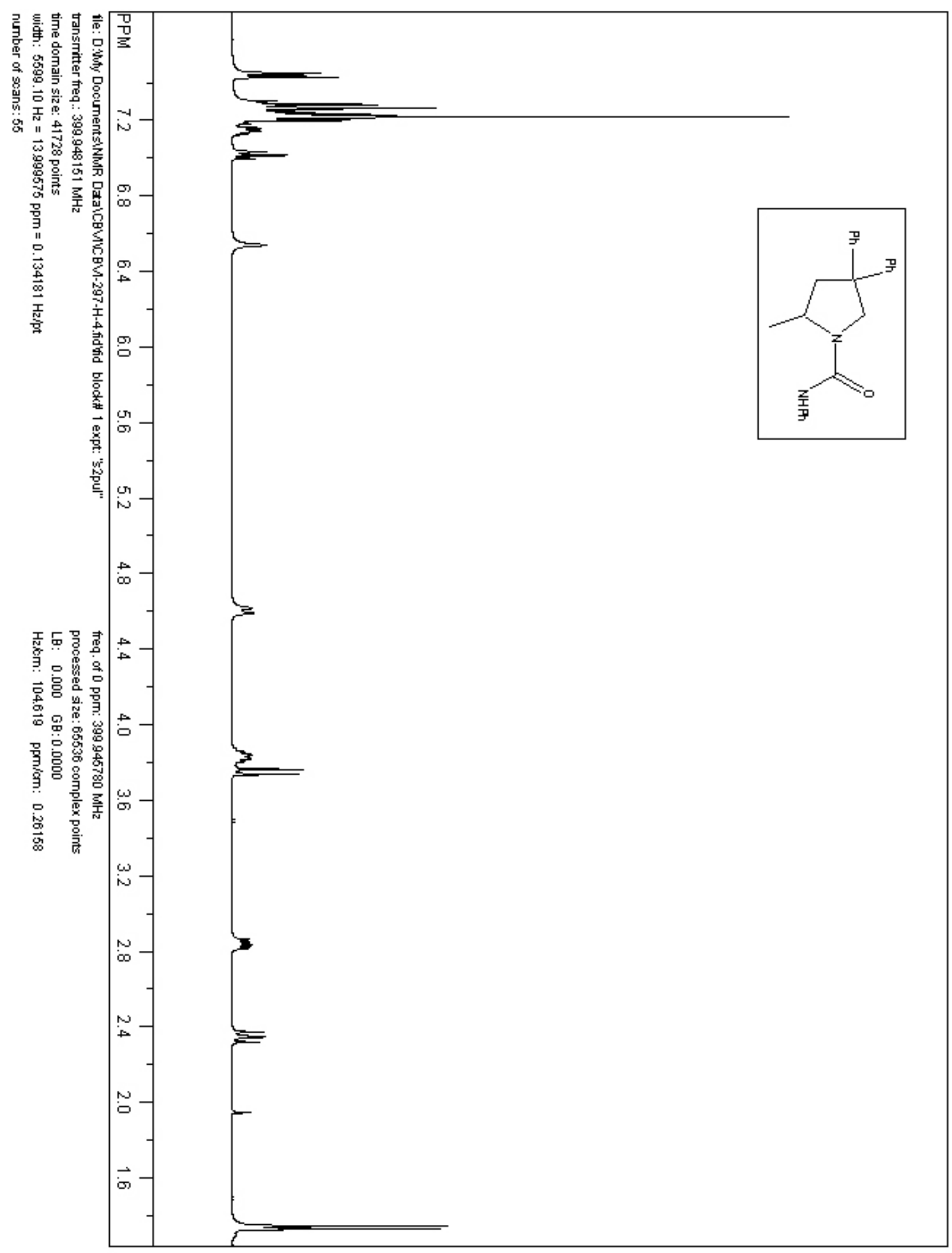


Figure S9. ${ }^{13} \mathrm{C}\left\{{ }^{1} \mathrm{H}\right\}$ NMR spectrum of $\mathbf{3 c}$.

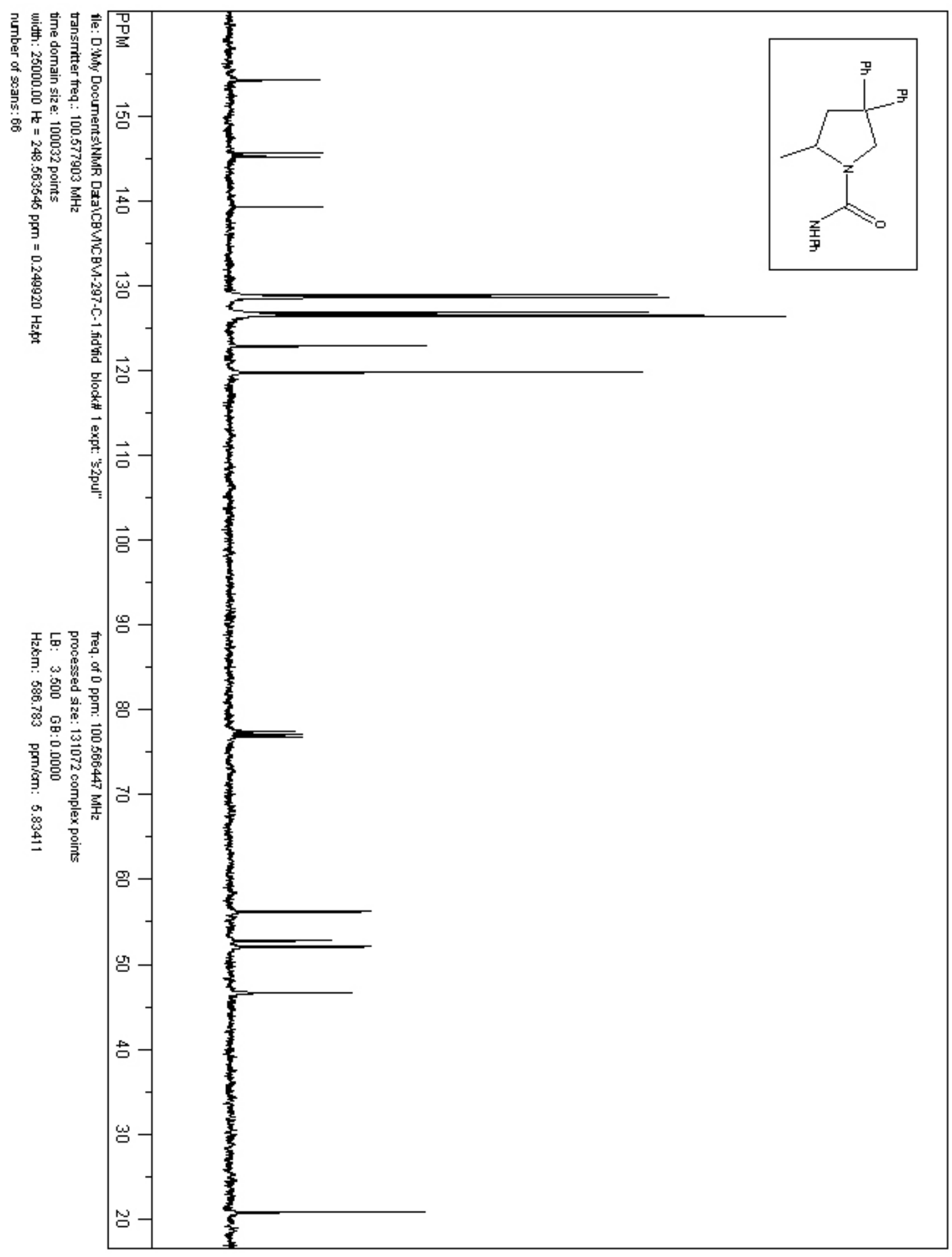


Figure S10. ${ }^{1} \mathrm{H}$ NMR spectrum of $\mathbf{3 b}$.

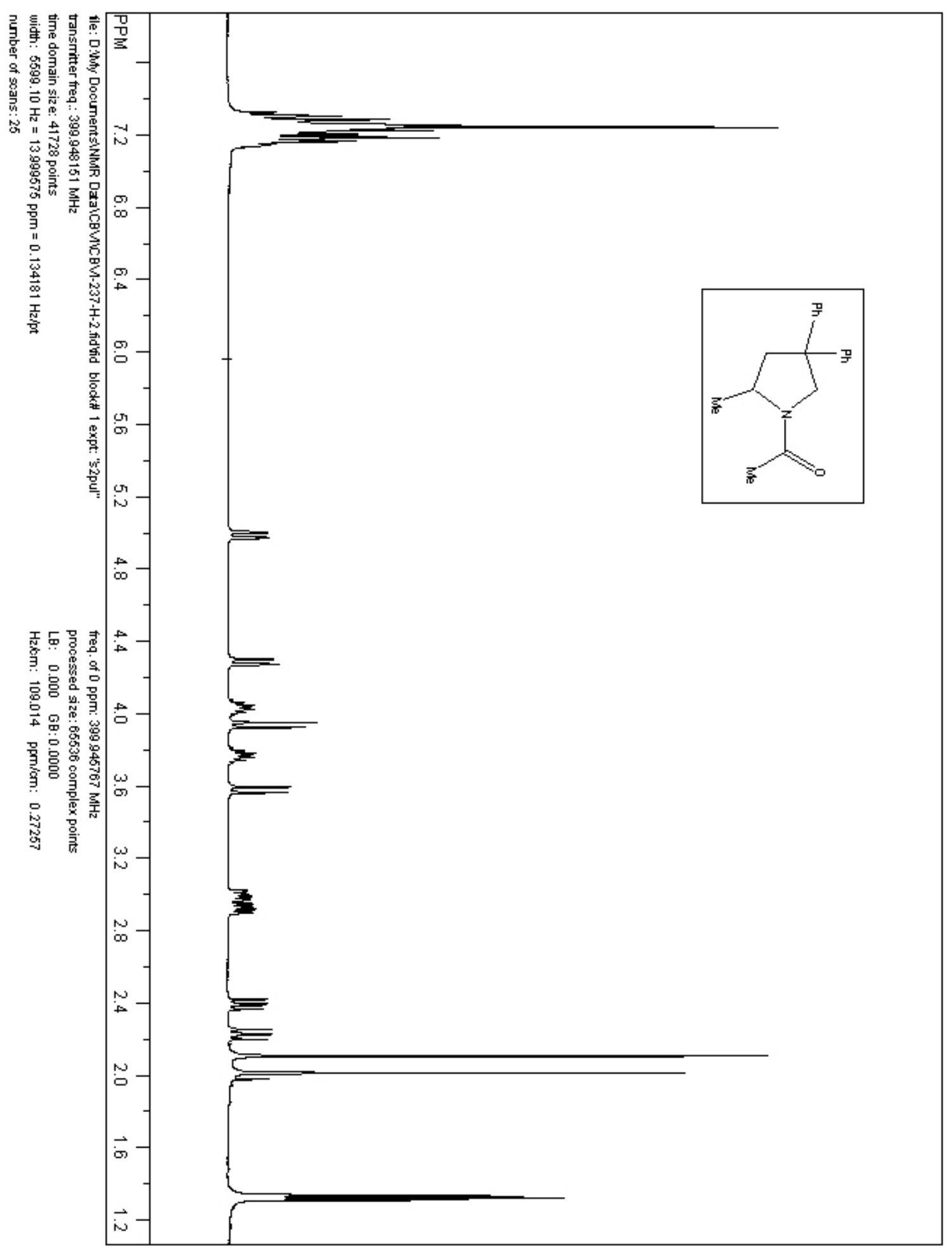


Figure S11. ${ }^{13} \mathrm{C}\left\{{ }^{1} \mathrm{H}\right\}$ NMR spectrum of $\mathbf{3 b}$.

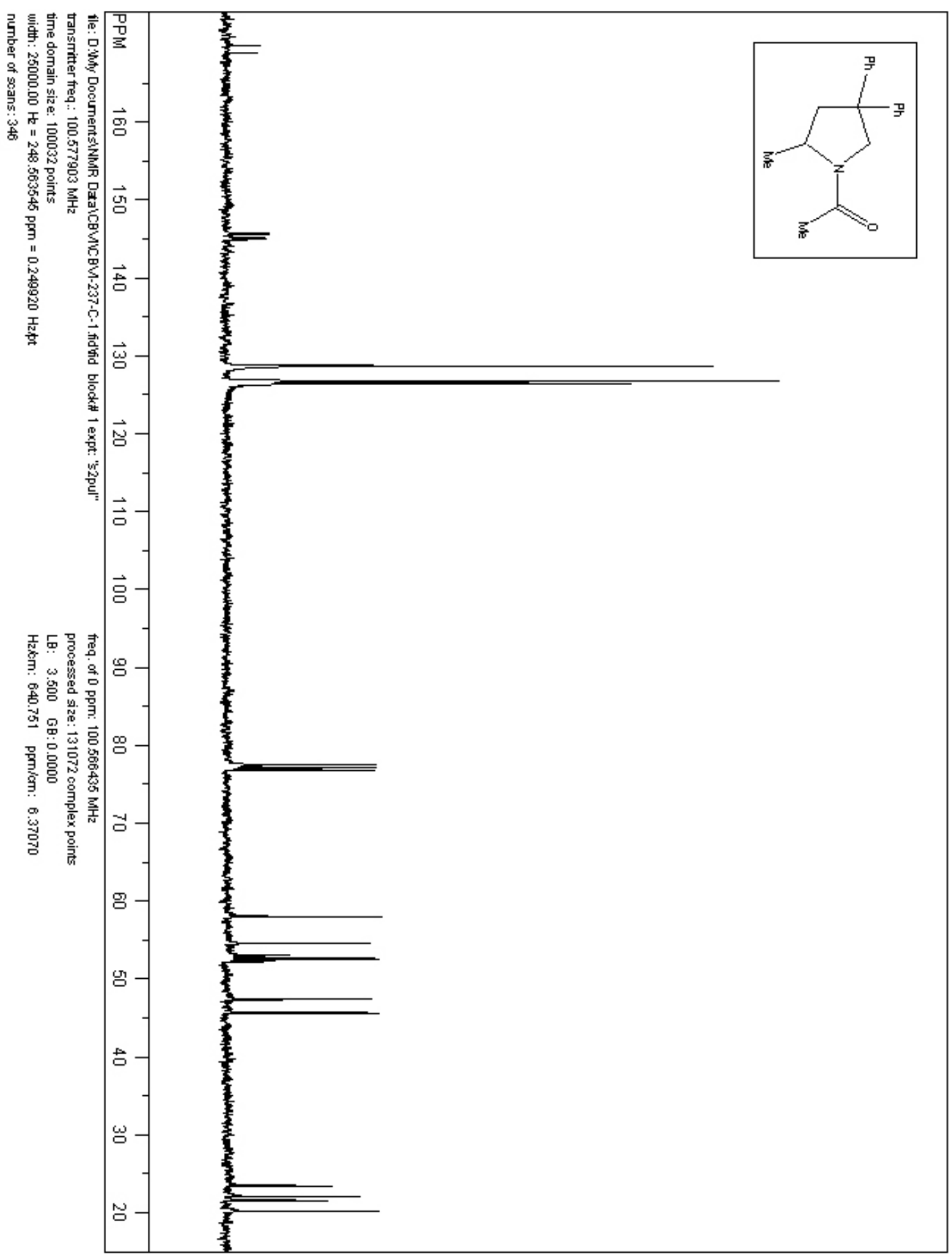


Figure S12. ${ }^{1} \mathrm{H}$ NMR spectrum of $\mathbf{3 a}$.

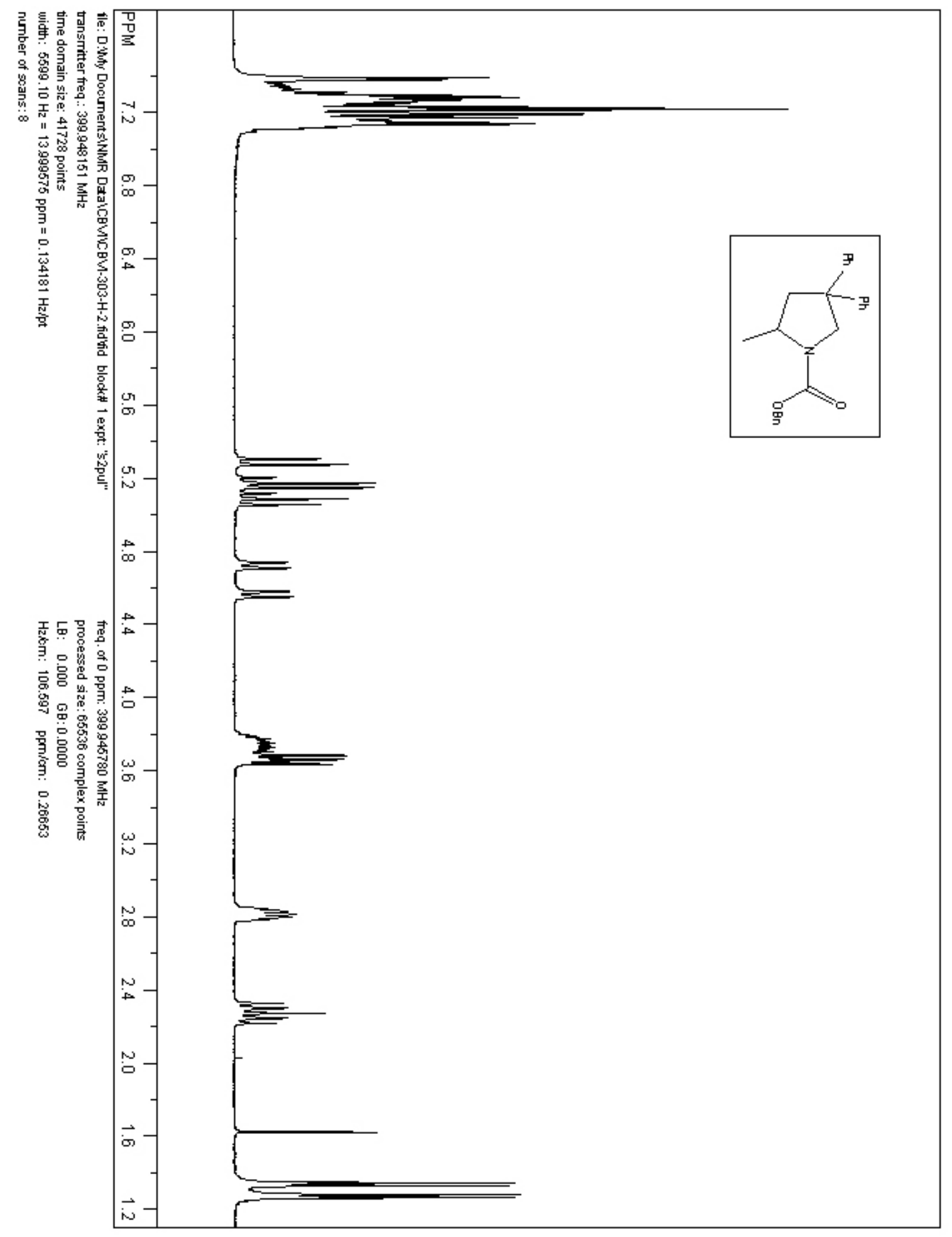


Figure S13. ${ }^{13} \mathrm{C}\left\{{ }^{1} \mathrm{H}\right\}$ NMR spectrum of $\mathbf{3 a}$.

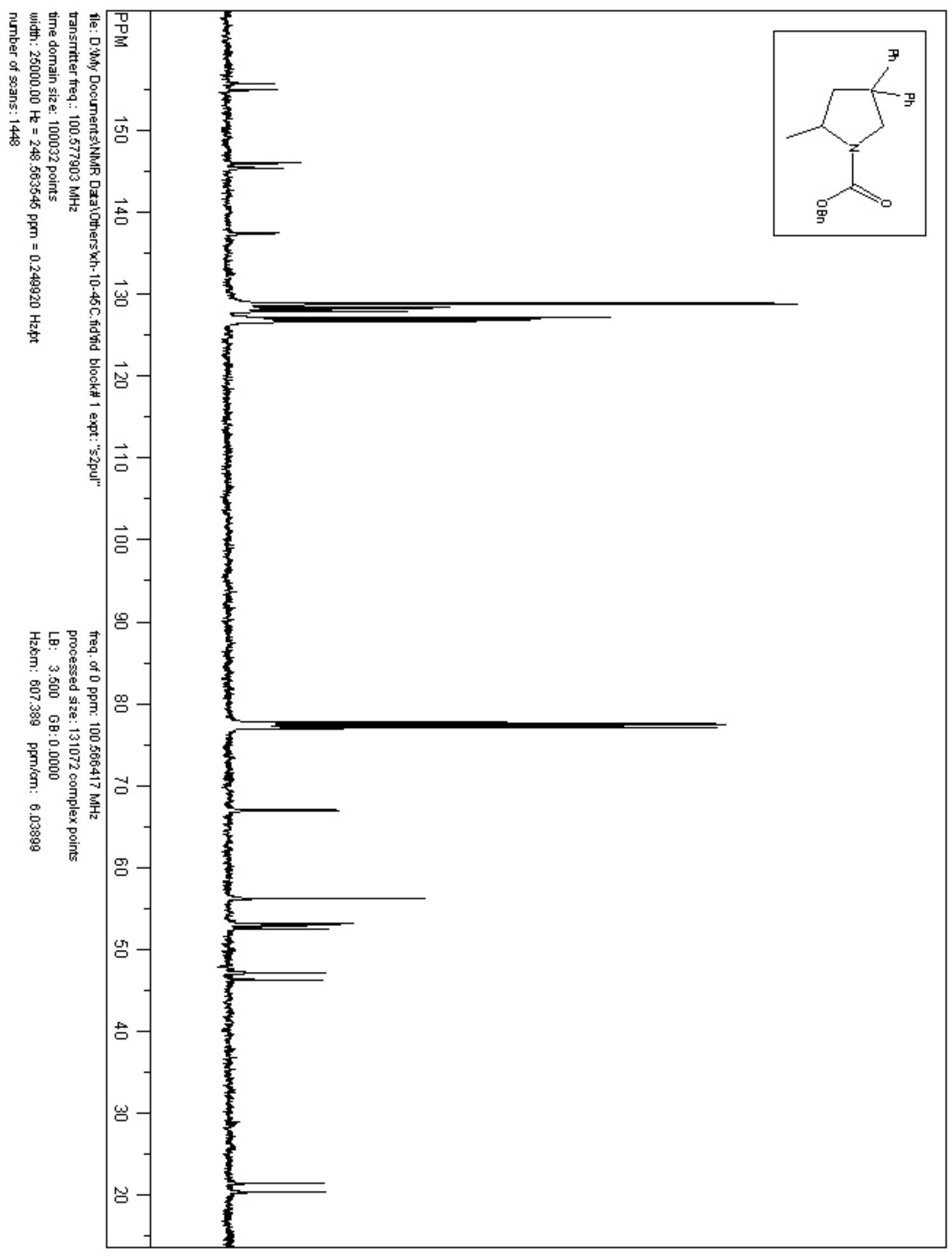


Figure S14. ${ }^{1}$ H NMR spectrum of S26.

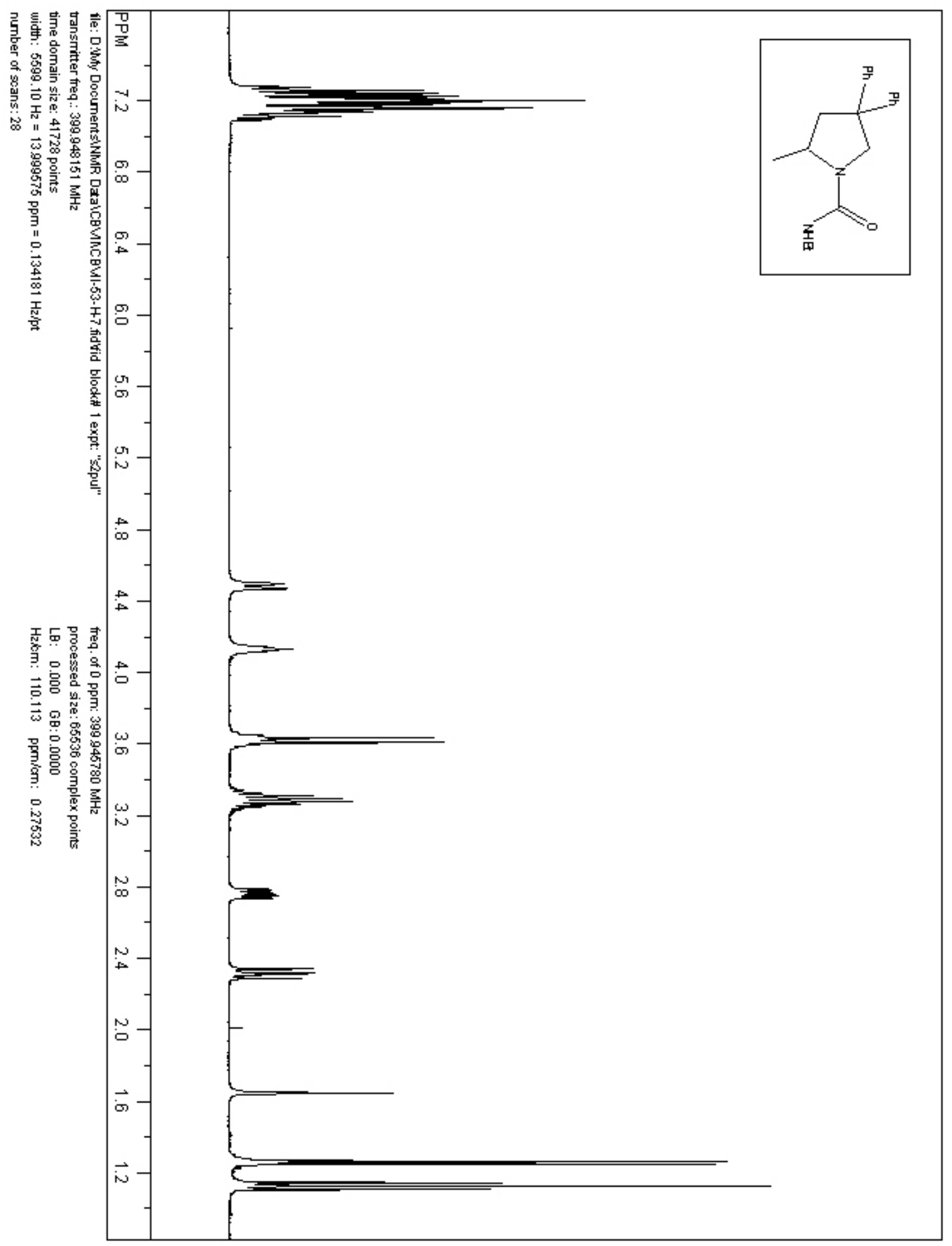

S30 
Figure S15. ${ }^{13} \mathrm{C}\left\{{ }^{1} \mathrm{H}\right\}$ NMR spectrum of $\mathbf{S 2 6}$.

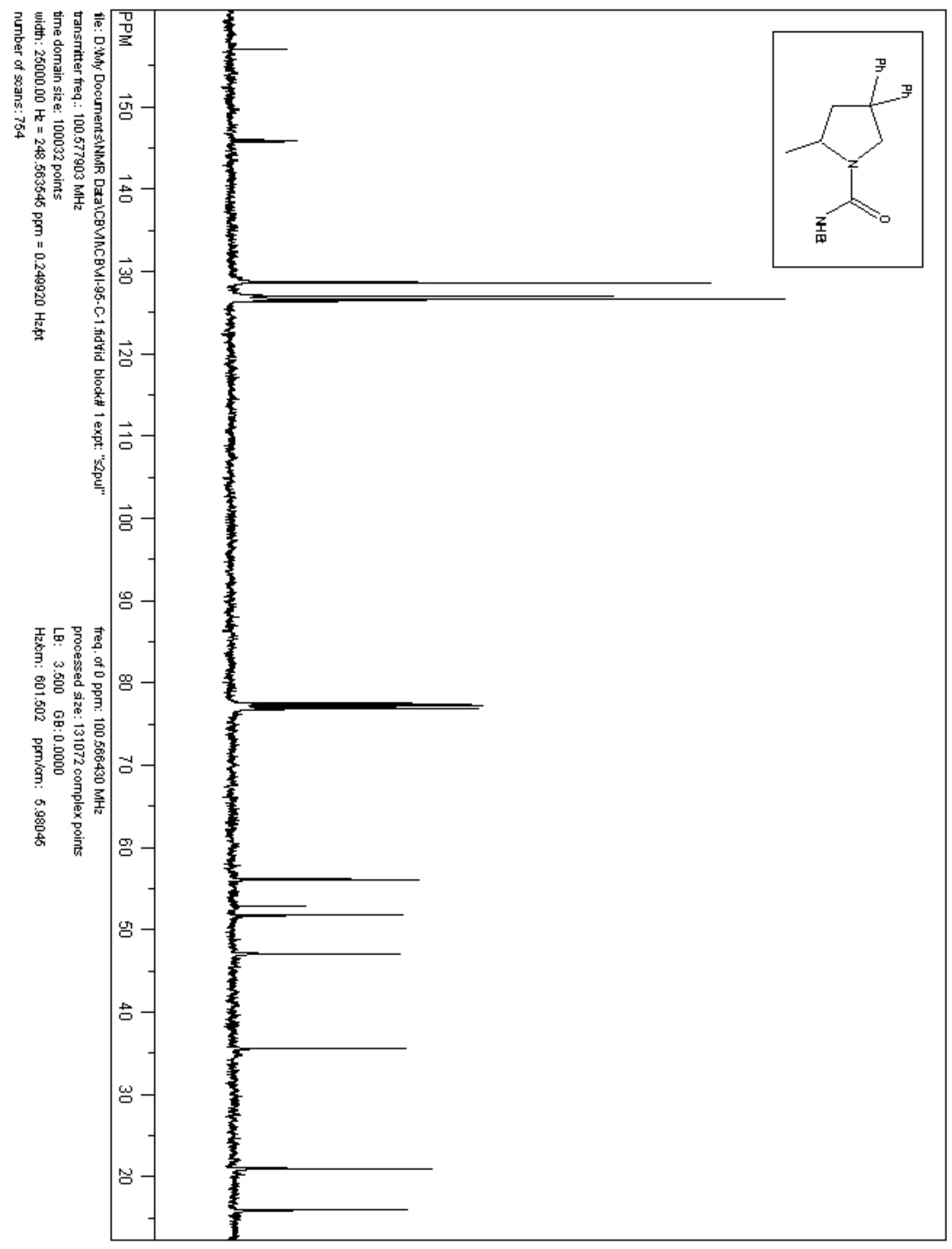


Figure S16. ${ }^{1} \mathrm{H}$ NMR spectrum of $\mathbf{S 2 7}$.

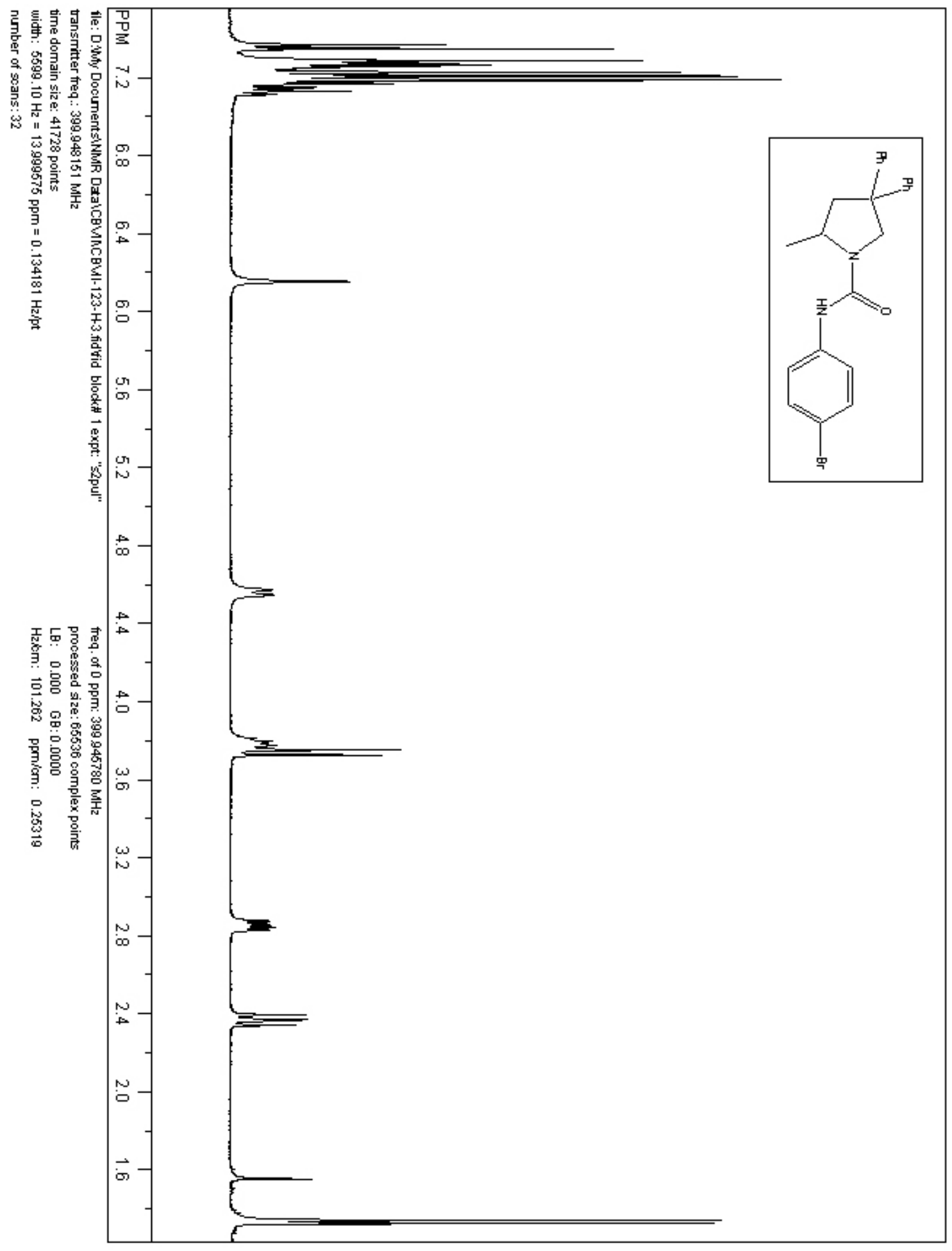


Figure S17. ${ }^{13} \mathrm{C}\left\{{ }^{1} \mathrm{H}\right\}$ NMR spectrum of $\mathbf{S 2 7}$.

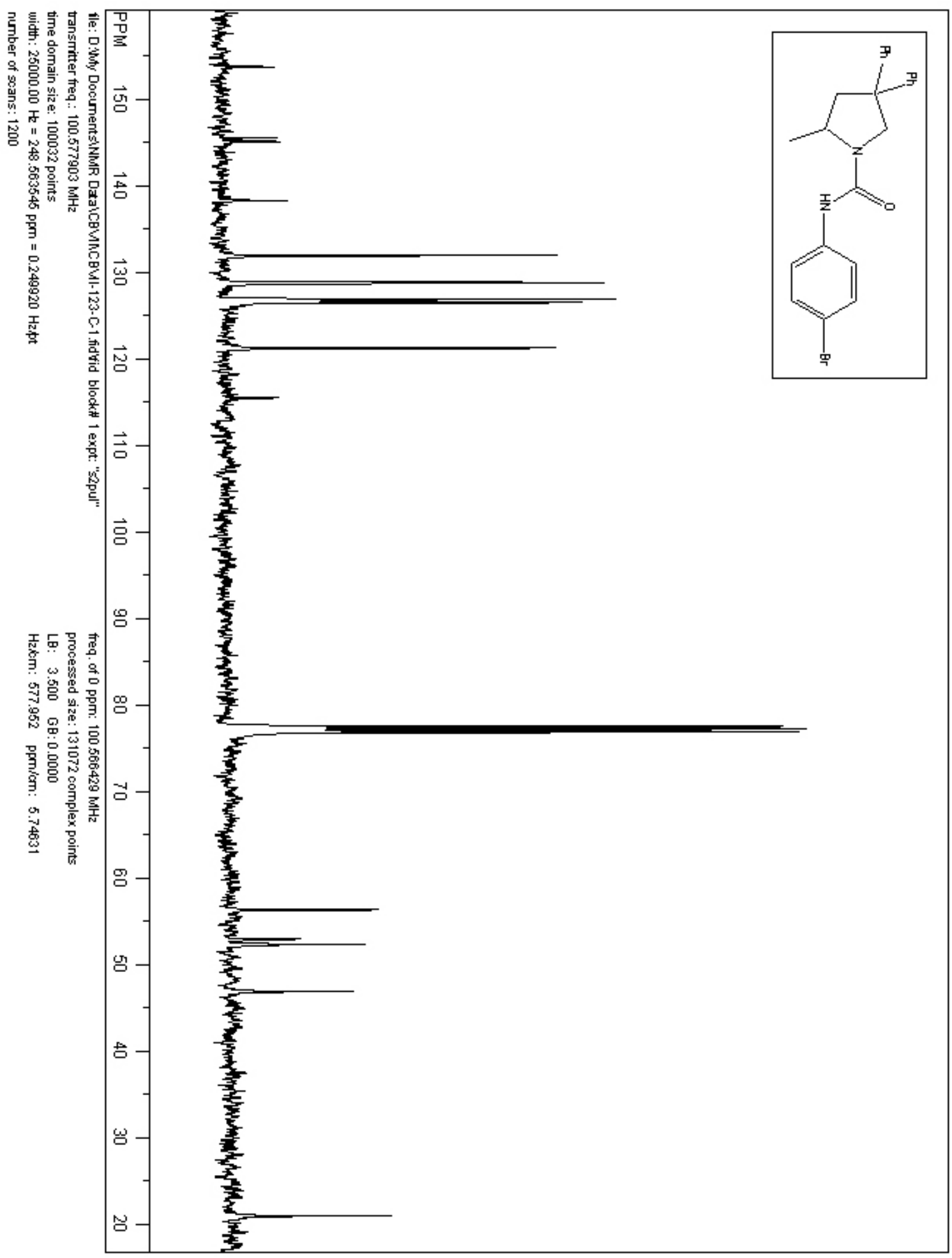


Figure S18. ${ }^{1} \mathrm{H}$ NMR spectrum of $\mathbf{S 2 8}$.

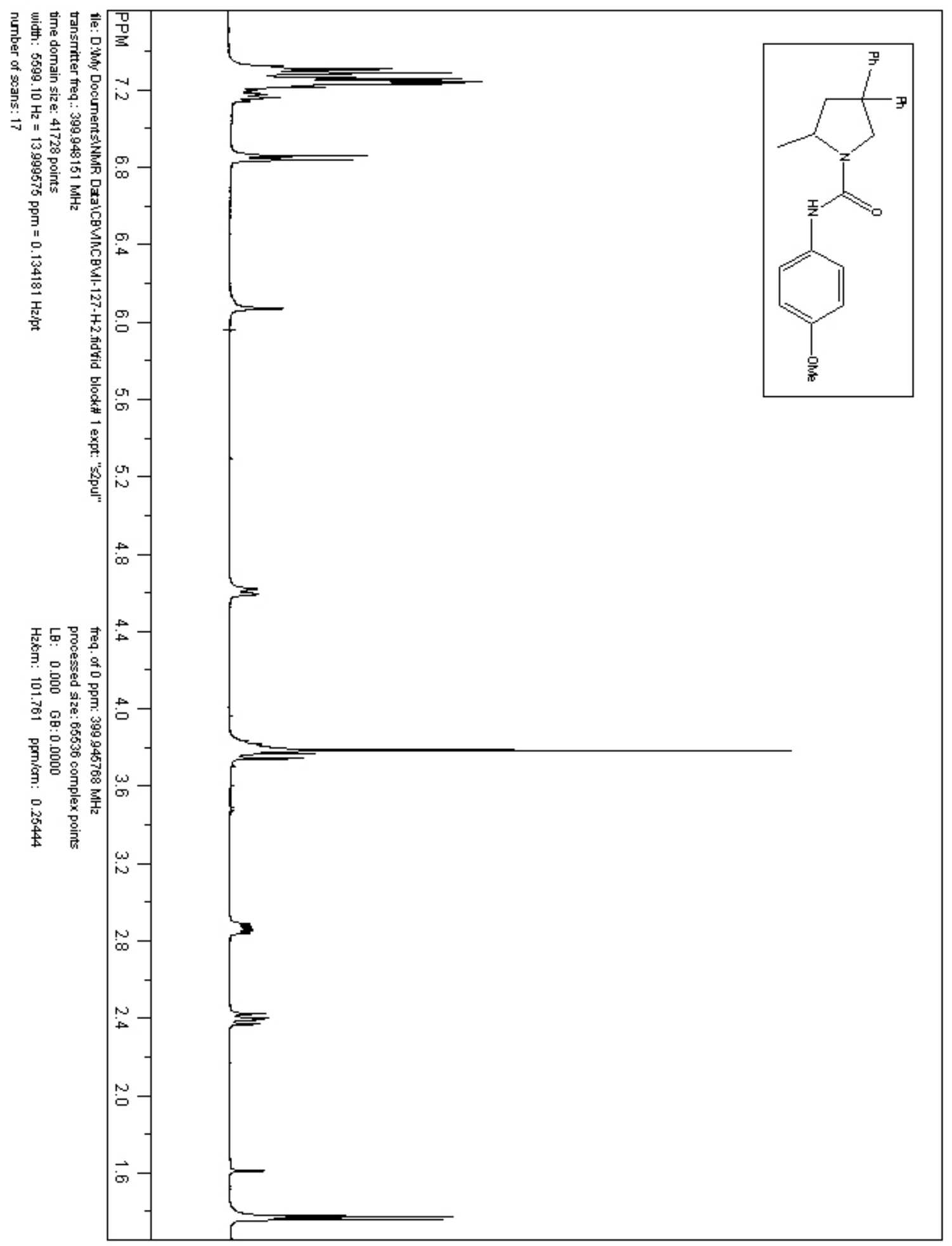


Figure S19. ${ }^{13} \mathrm{C}\left\{{ }^{1} \mathrm{H}\right\}$ NMR spectrum of $\mathbf{S 2 8}$.

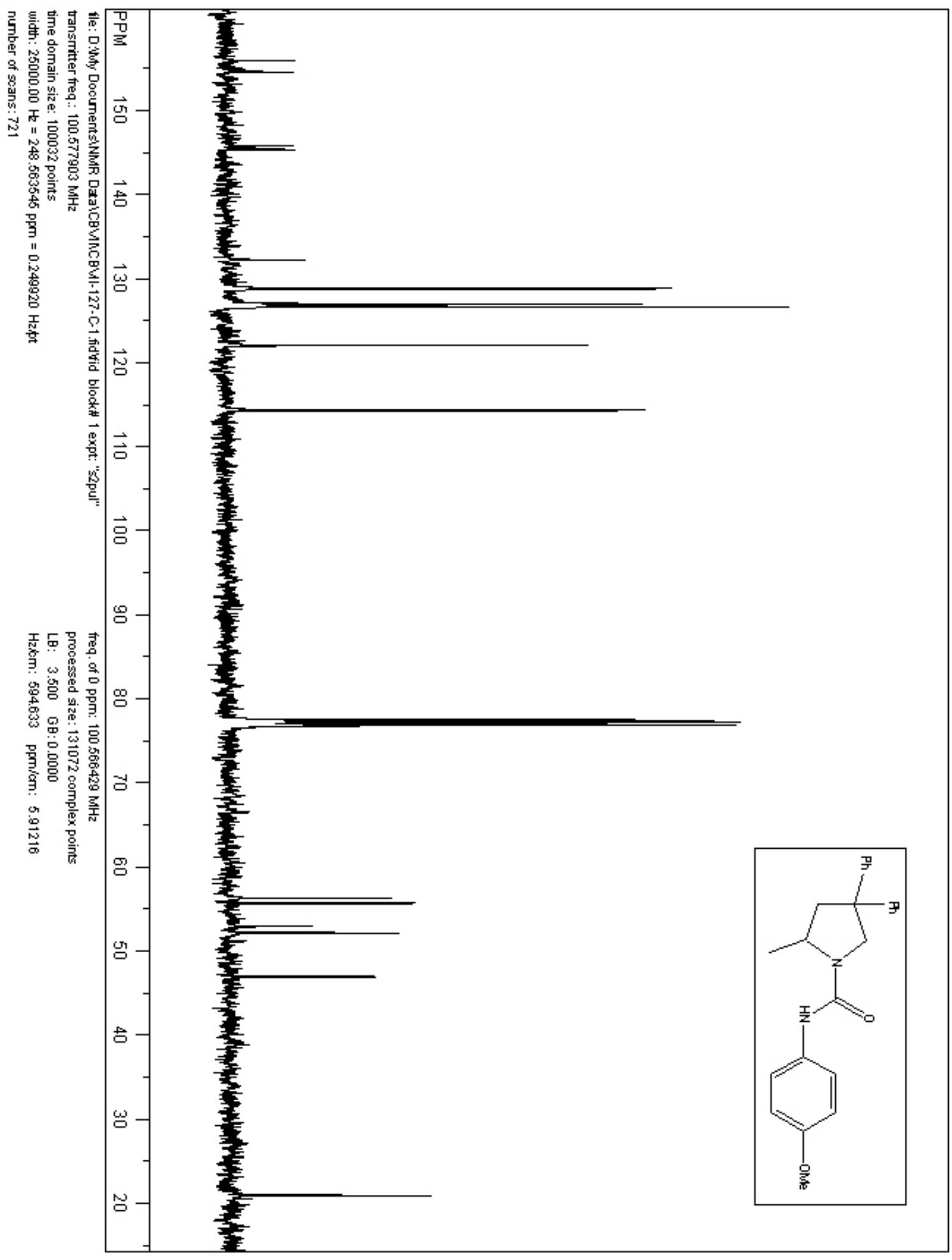


Figure S20. ${ }^{1}$ H NMR spectrum of $\mathbf{S 2 9}$.

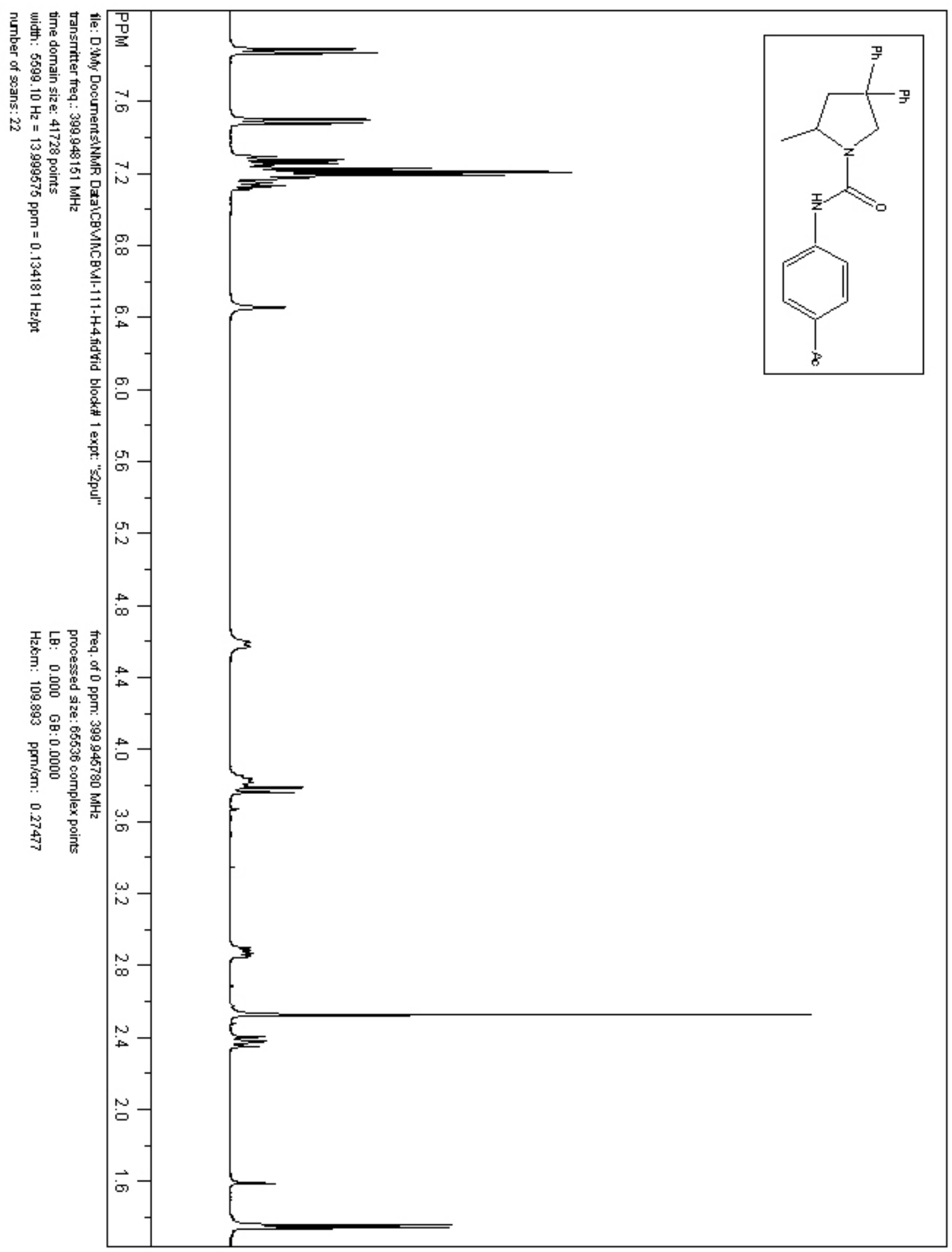


Figure S21. ${ }^{13} \mathrm{C}\left\{{ }^{1} \mathrm{H}\right\}$ NMR spectrum of $\mathbf{S 2 9}$.

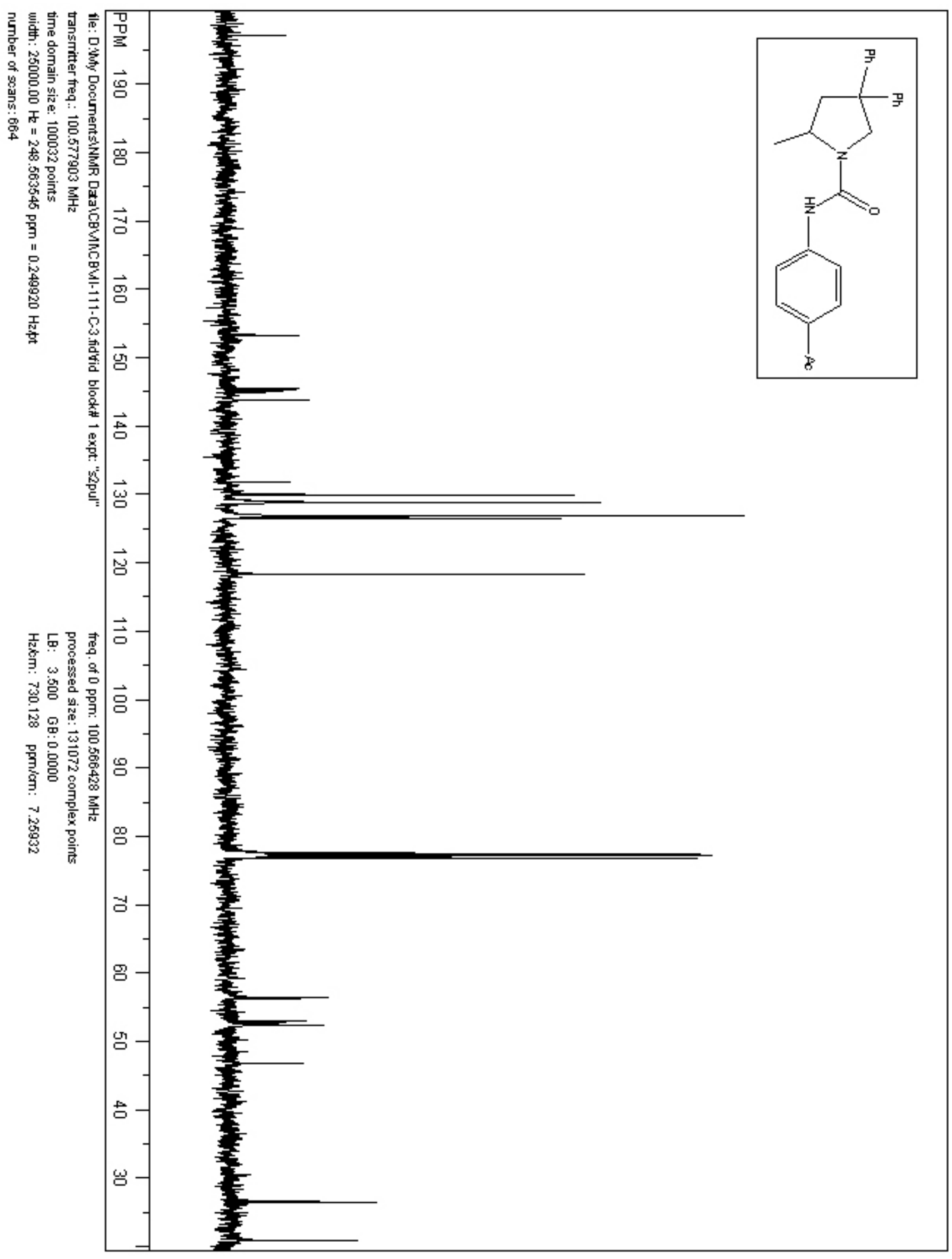


Figure S22. ${ }^{1} \mathrm{H}$ NMR spectrum of $\mathbf{S 3 0}$.

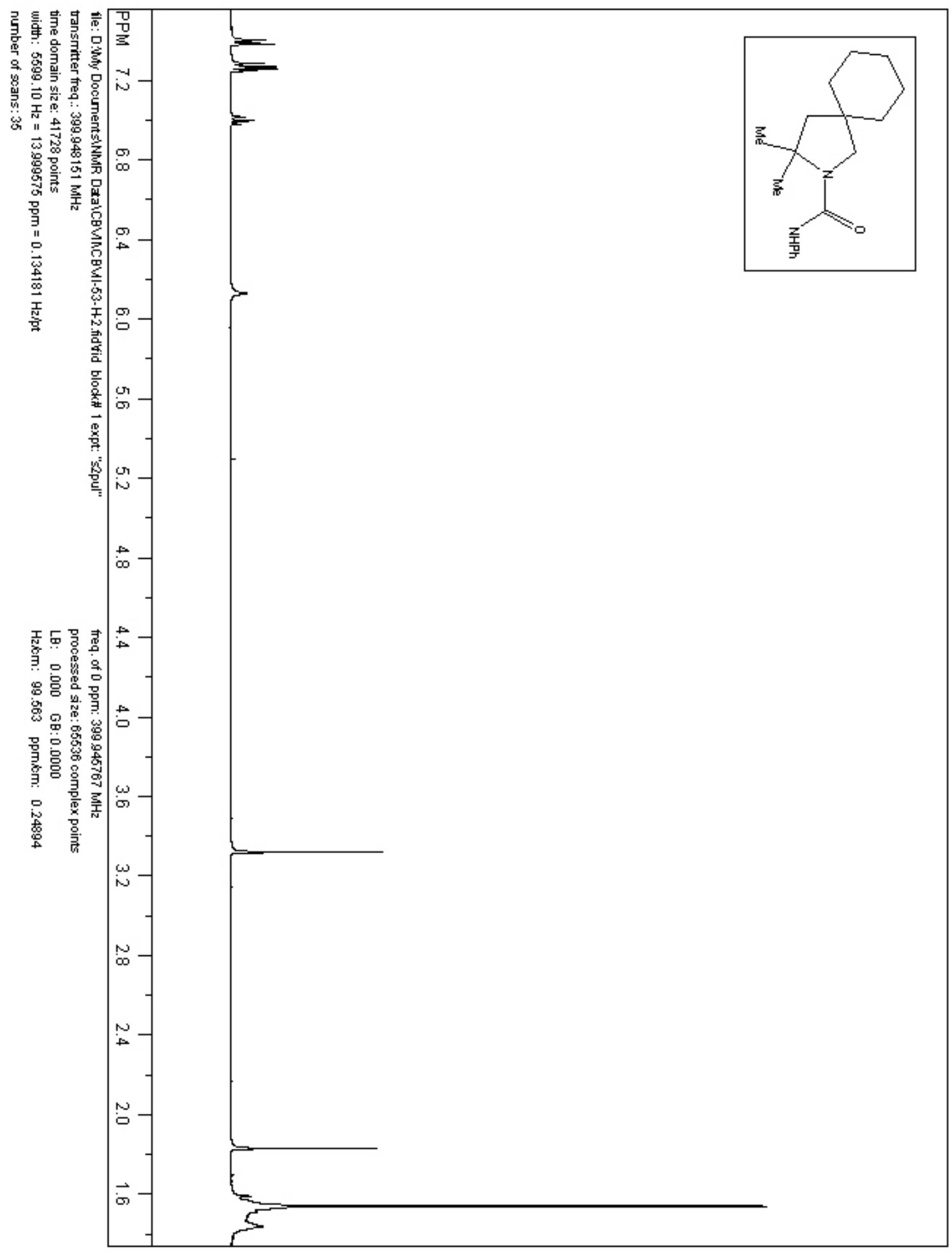


Figure S23. ${ }^{13} \mathrm{C}\left\{{ }^{1} \mathrm{H}\right\}$ NMR spectrum of $\mathbf{S 3 0}$.

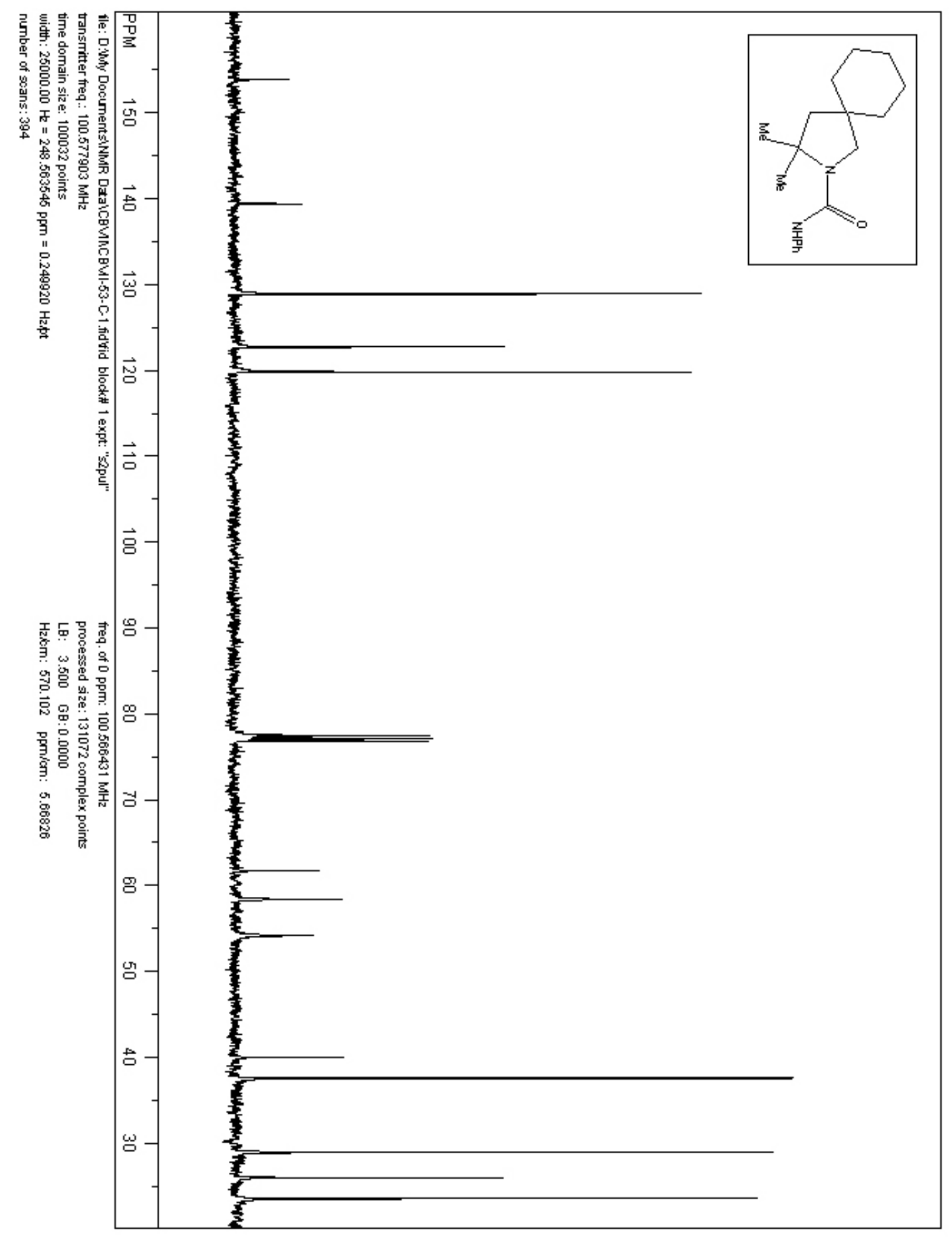


Figure S24. ${ }^{1} \mathrm{H}$ NMR spectrum (14:1-cis:trans) S31.

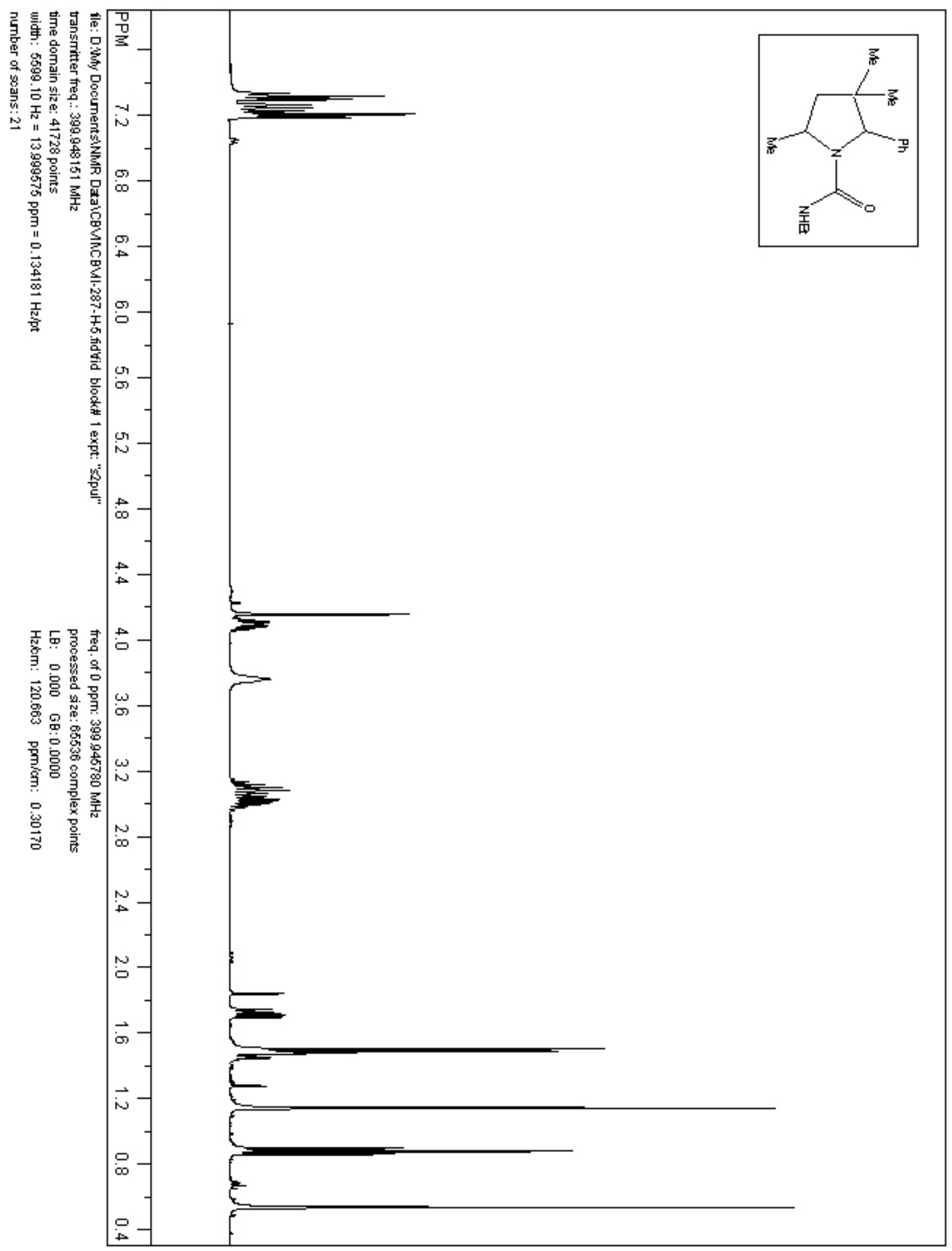


Figure S25. ${ }^{13} \mathrm{C}\left\{{ }^{1} \mathrm{H}\right\}$ NMR (>20:1-cis:trans) spectrum of $\mathbf{S 3 1}$.

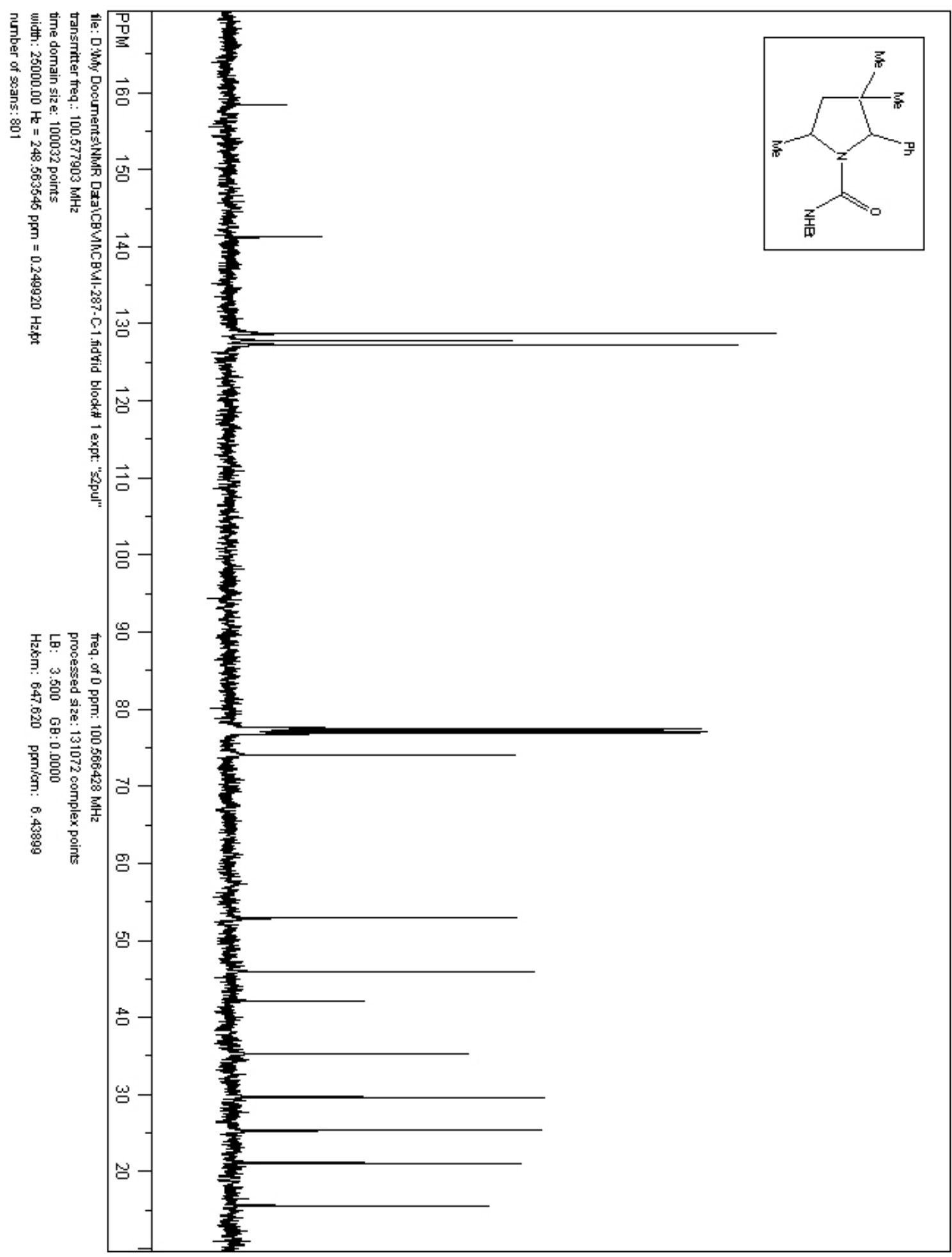


Figure S26. ${ }^{1} \mathrm{H}$ NMR spectrum of the cis-diastereomer of $\mathbf{S 3 2}$.

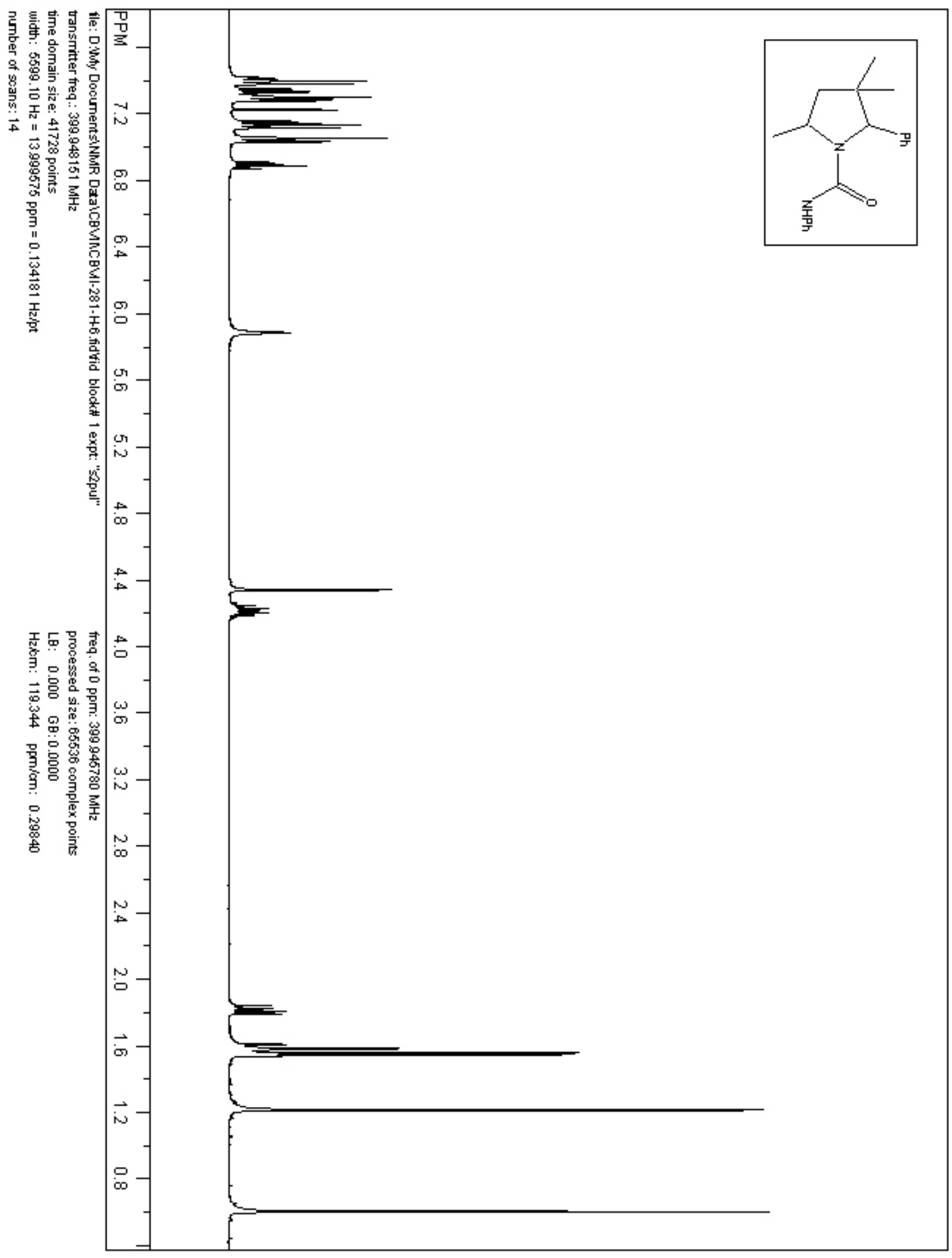


Figure S27. ${ }^{13} \mathrm{C}\left\{{ }^{1} \mathrm{H}\right\}$ NMR spectrum of cis-diastereomer of $\mathbf{S 3 2}$.

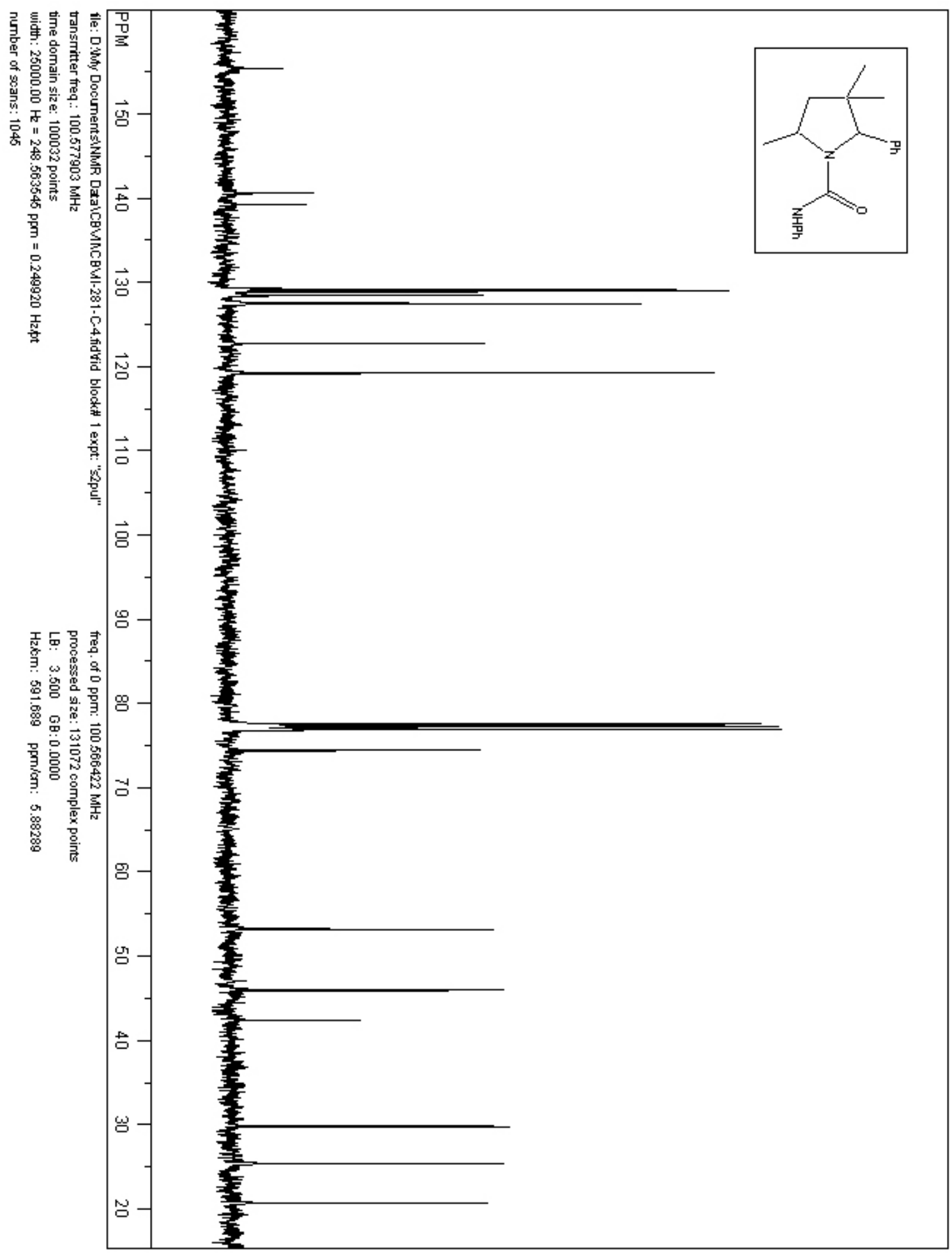


Figure S28. ${ }^{1}$ H NMR (3.7:1-cis:trans) spectrum of $\mathbf{S 3 3}$.

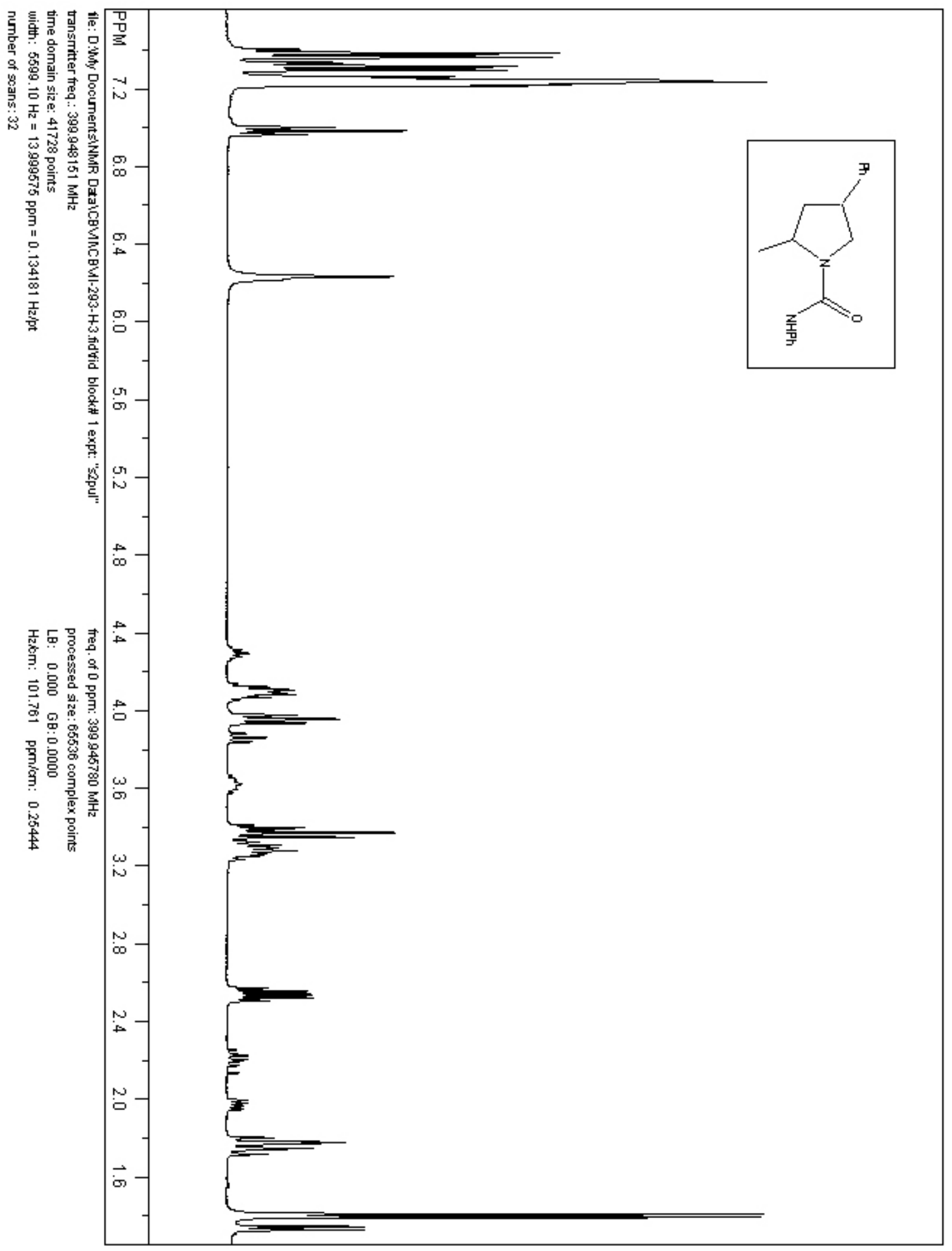


Figure S29. ${ }^{13} \mathrm{C}\left\{{ }^{1} \mathrm{H}\right\}$ NMR (3.7:1-cis:trans) spectrum of $\mathbf{S 3 3}$.

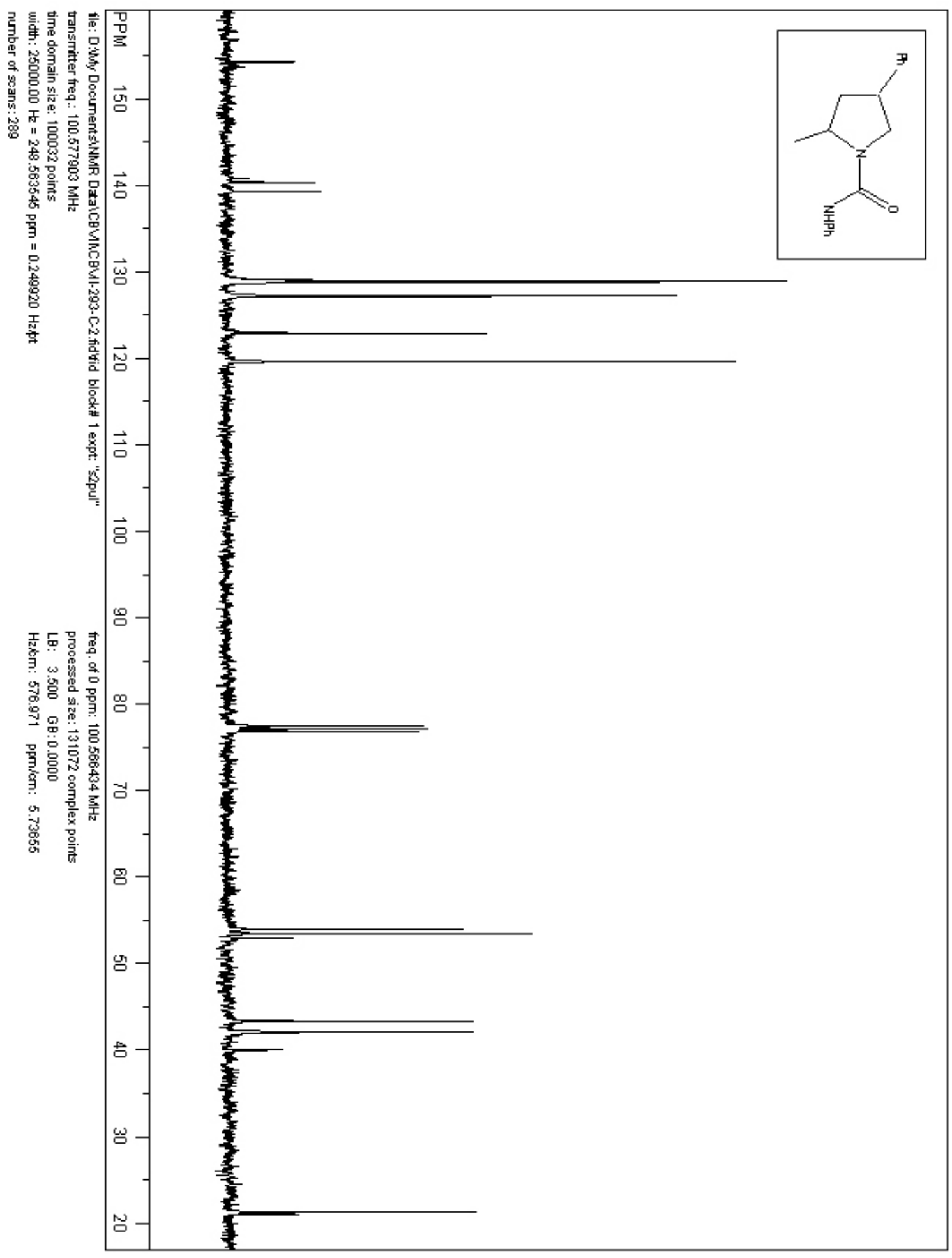


Figure S30. ${ }^{1}$ H NMR (3.0:1-cis:trans) spectrum of $\mathbf{S 3 4}$.

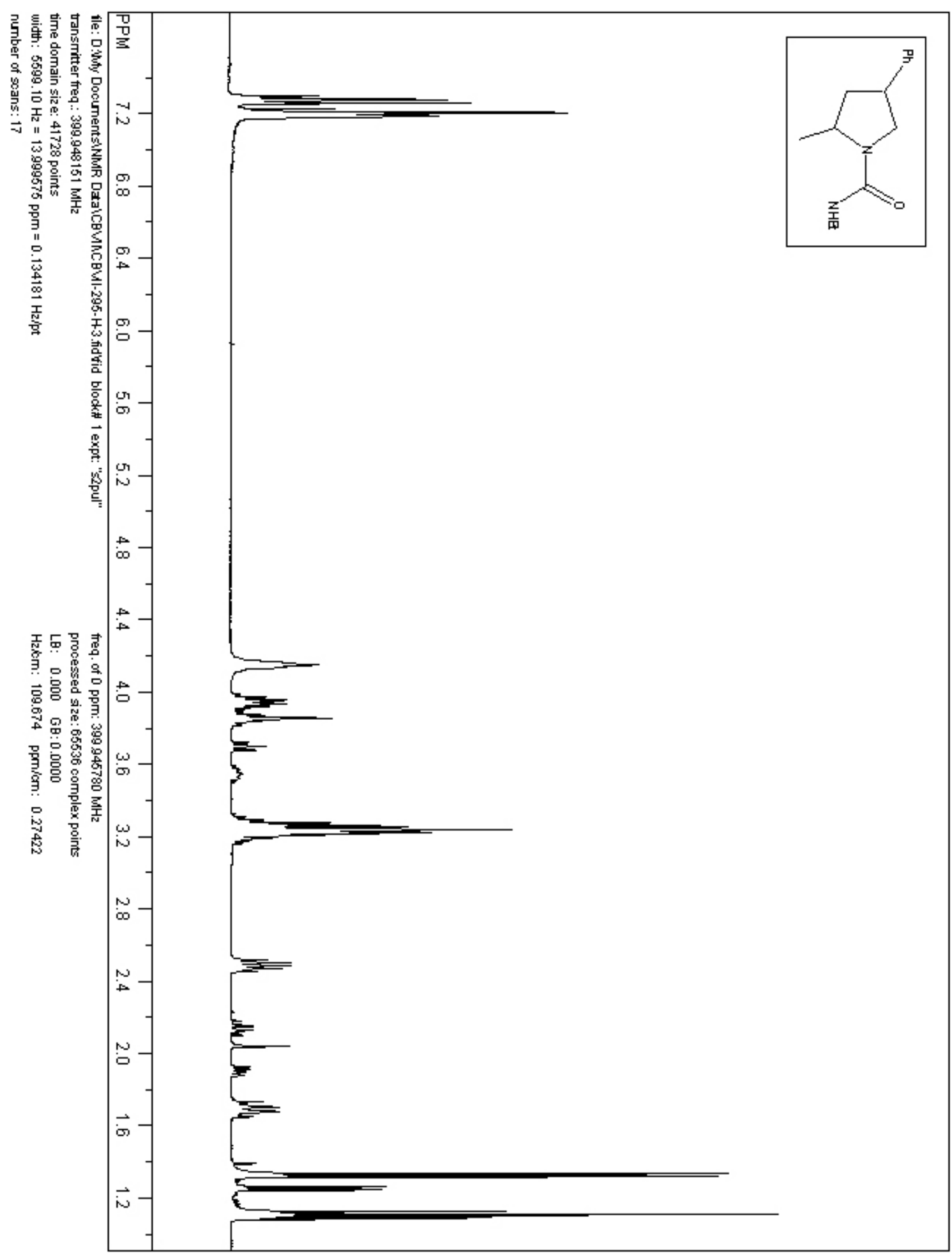


Figure S31. ${ }^{13} \mathrm{C}\left\{{ }^{1} \mathrm{H}\right\}$ NMR (3.0:1-cis:trans) spectrum of $\mathbf{S 3 4}$.

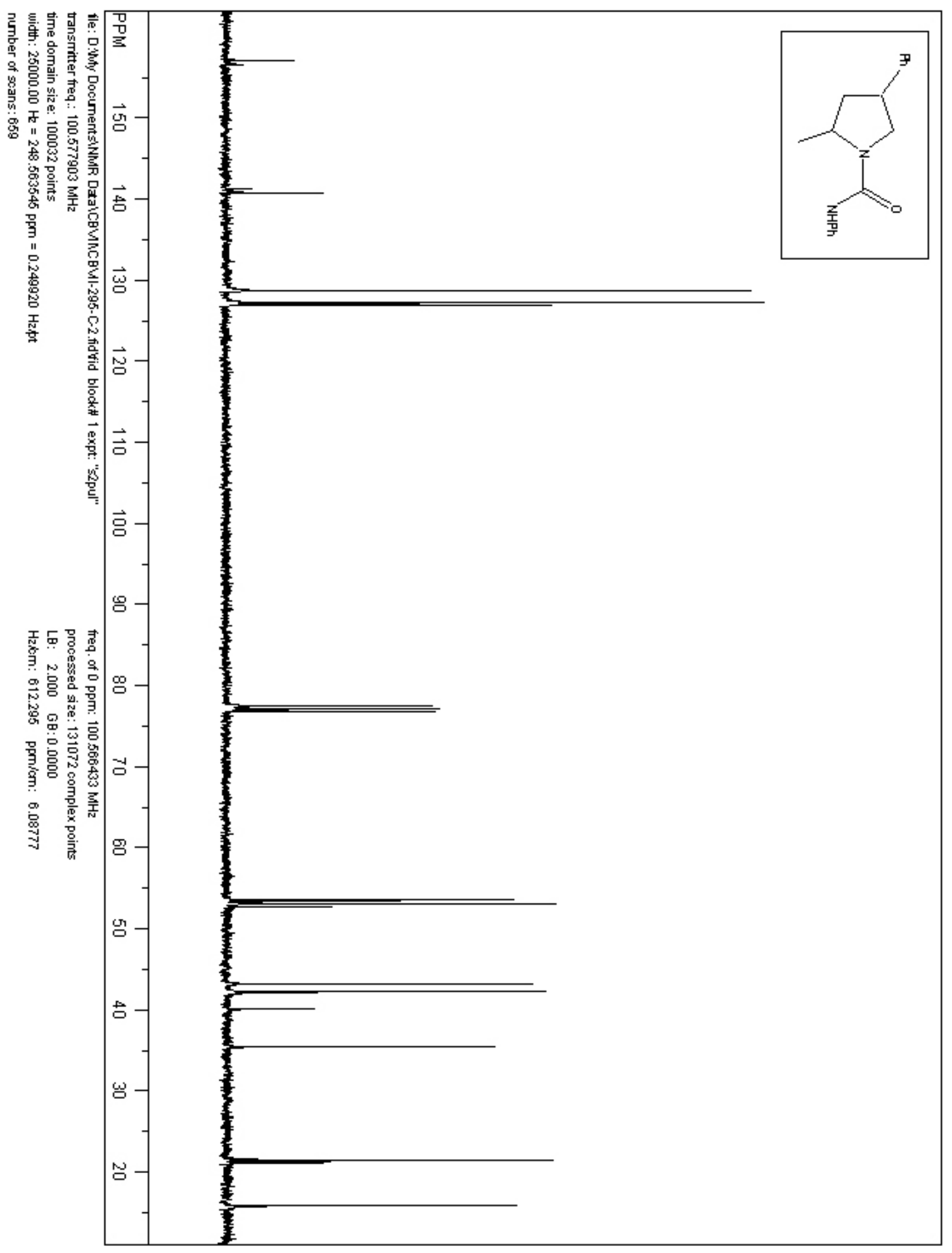


Figure S32. ${ }^{1}$ H NMR (4.1:1-cis:trans) spectrum of $\mathbf{S 3 5}$.

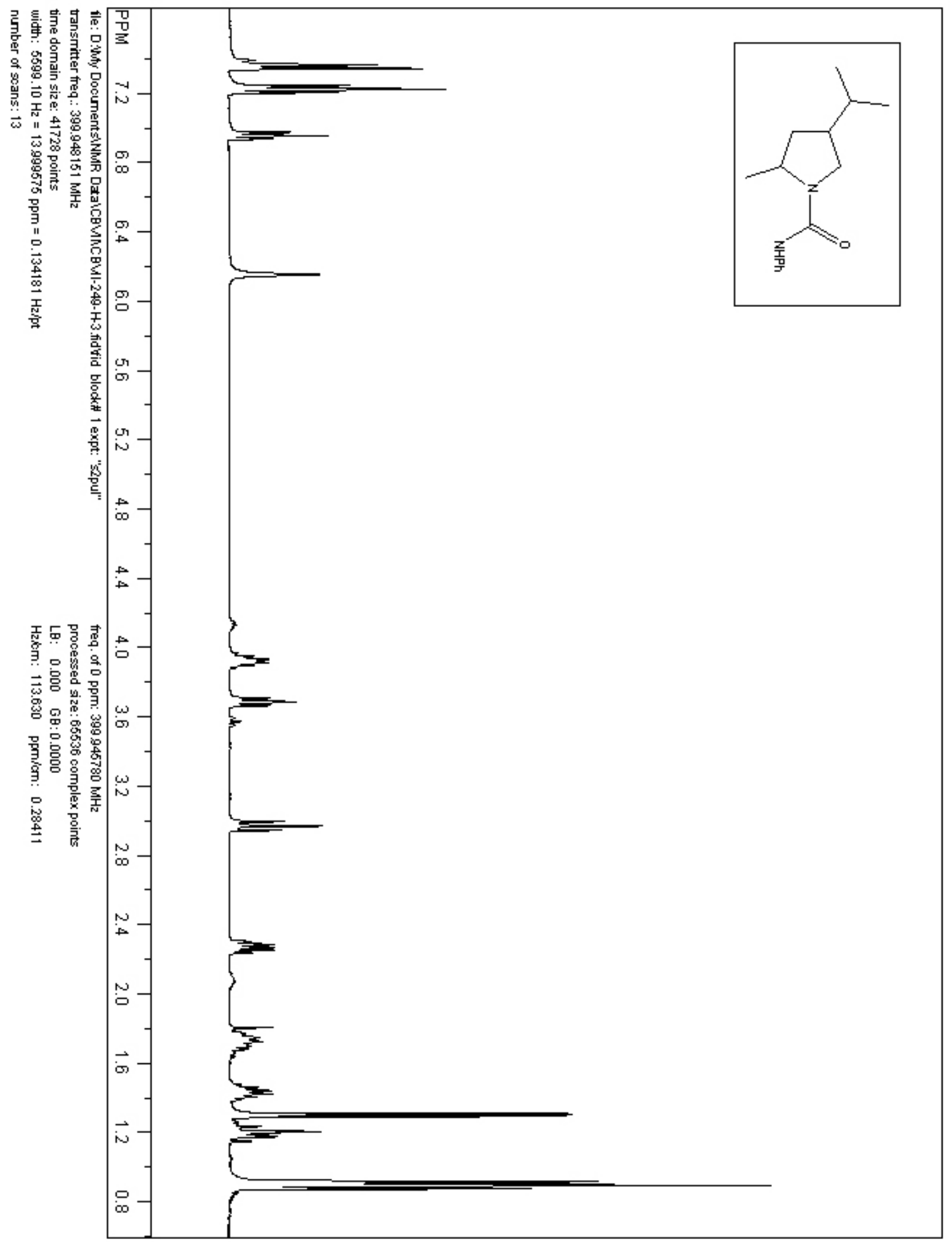


Figure S33. ${ }^{13} \mathrm{C}\left\{{ }^{1} \mathrm{H}\right\}$ (4.1:1-cis:trans) NMR spectrum of $\mathbf{S 3 5}$.

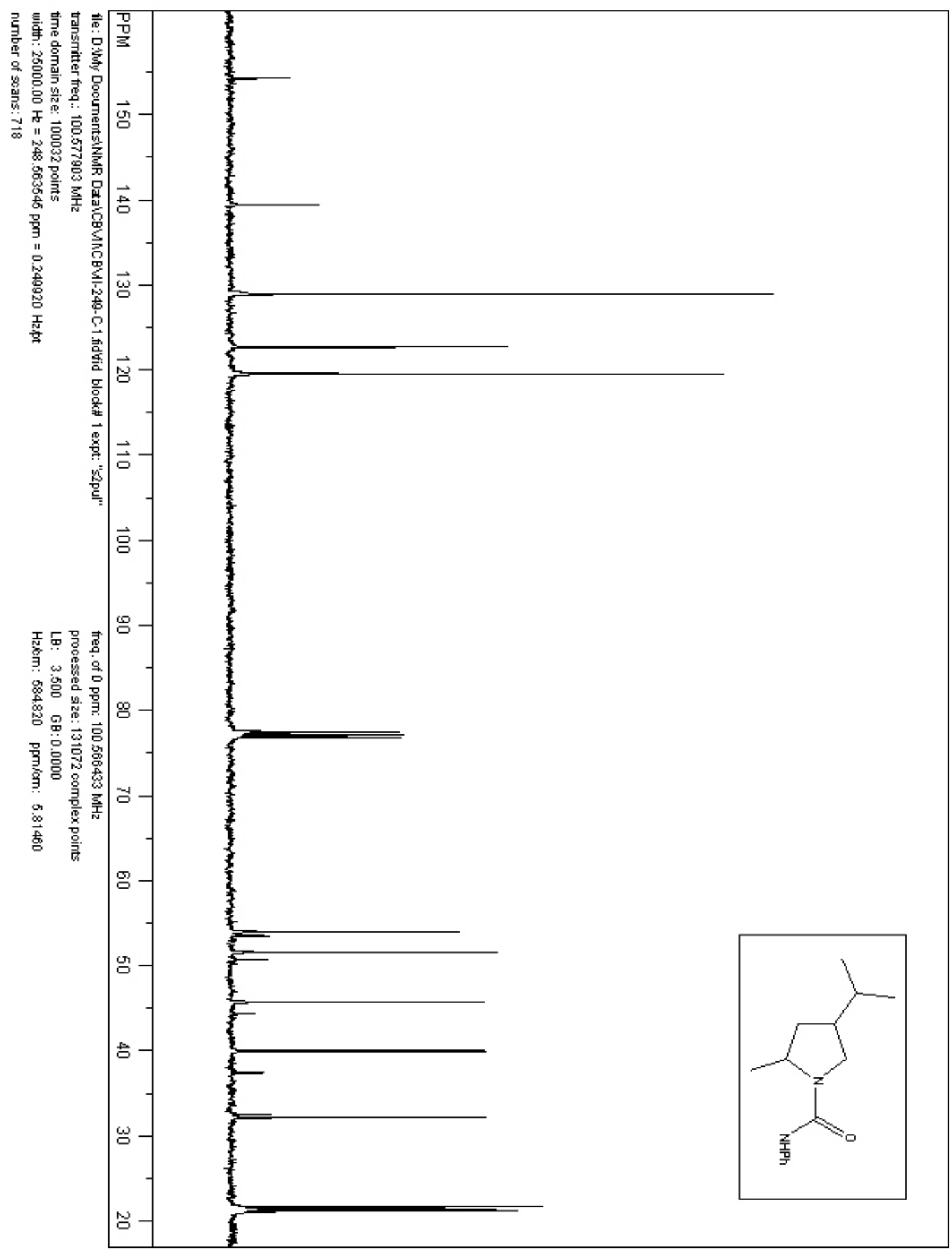


Figure S34. ${ }^{1} \mathrm{H}$ NMR spectrum of $\mathbf{S 3 6}$.

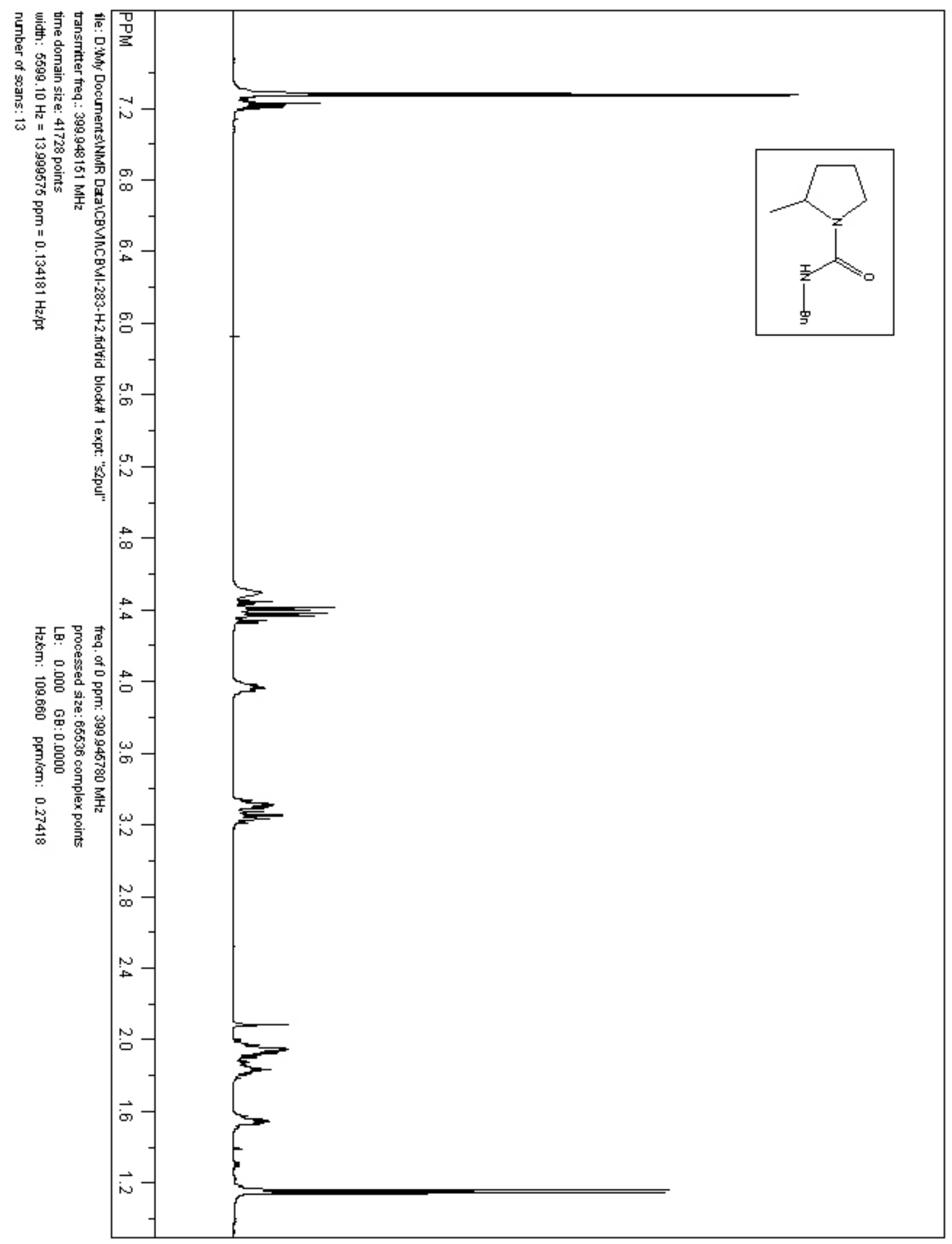


Figure S35. ${ }^{13} \mathrm{C}\left\{{ }^{1} \mathrm{H}\right\}$ NMR spectrum of $\mathbf{S 3 6}$.

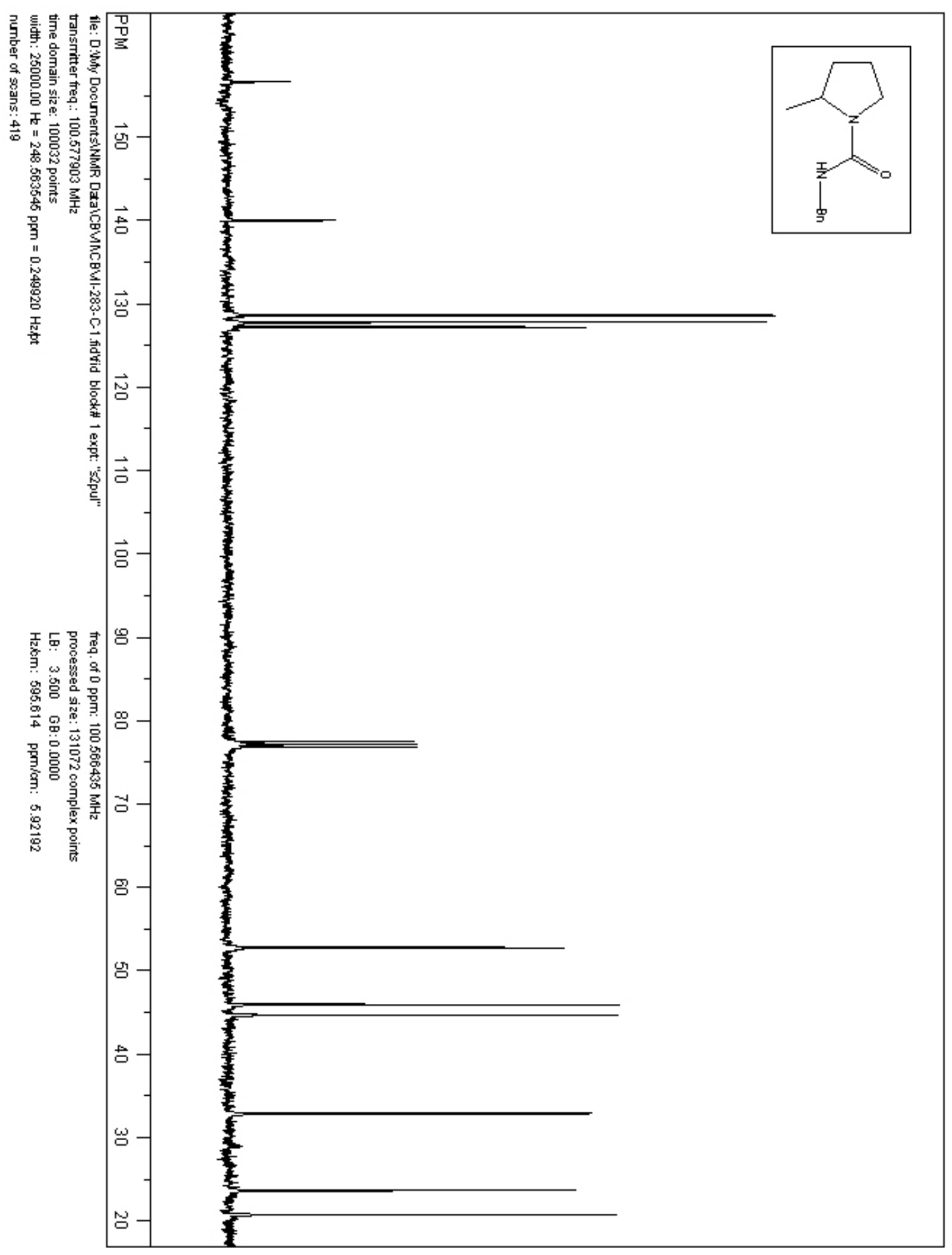

S51 
Figure S36. ${ }^{1}$ H NMR spectrum of $\mathbf{S 3 7}$.

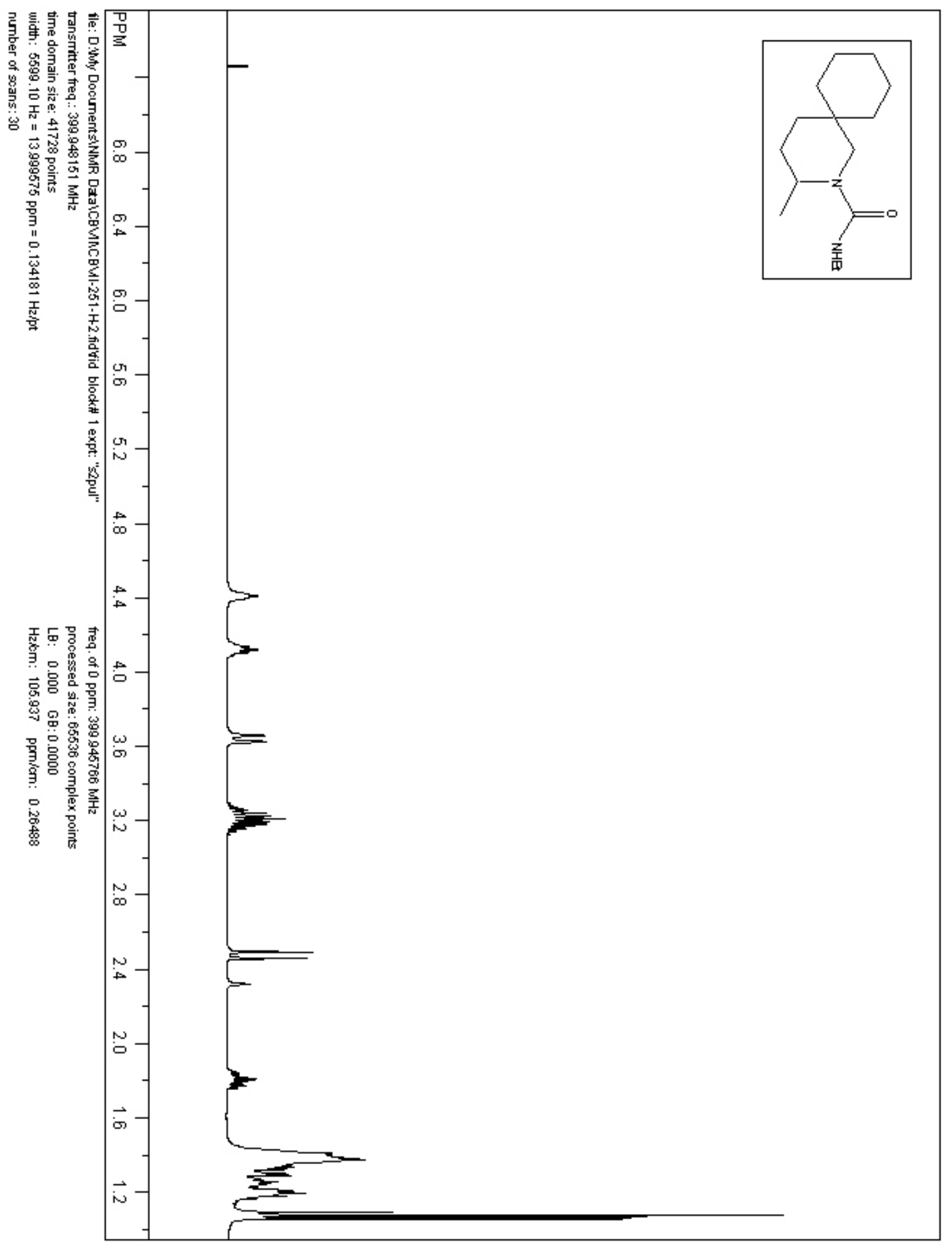


Figure S37. ${ }^{13} \mathrm{C}\left\{{ }^{1} \mathrm{H}\right\}$ NMR spectrum of $\mathbf{S 3 7}$.

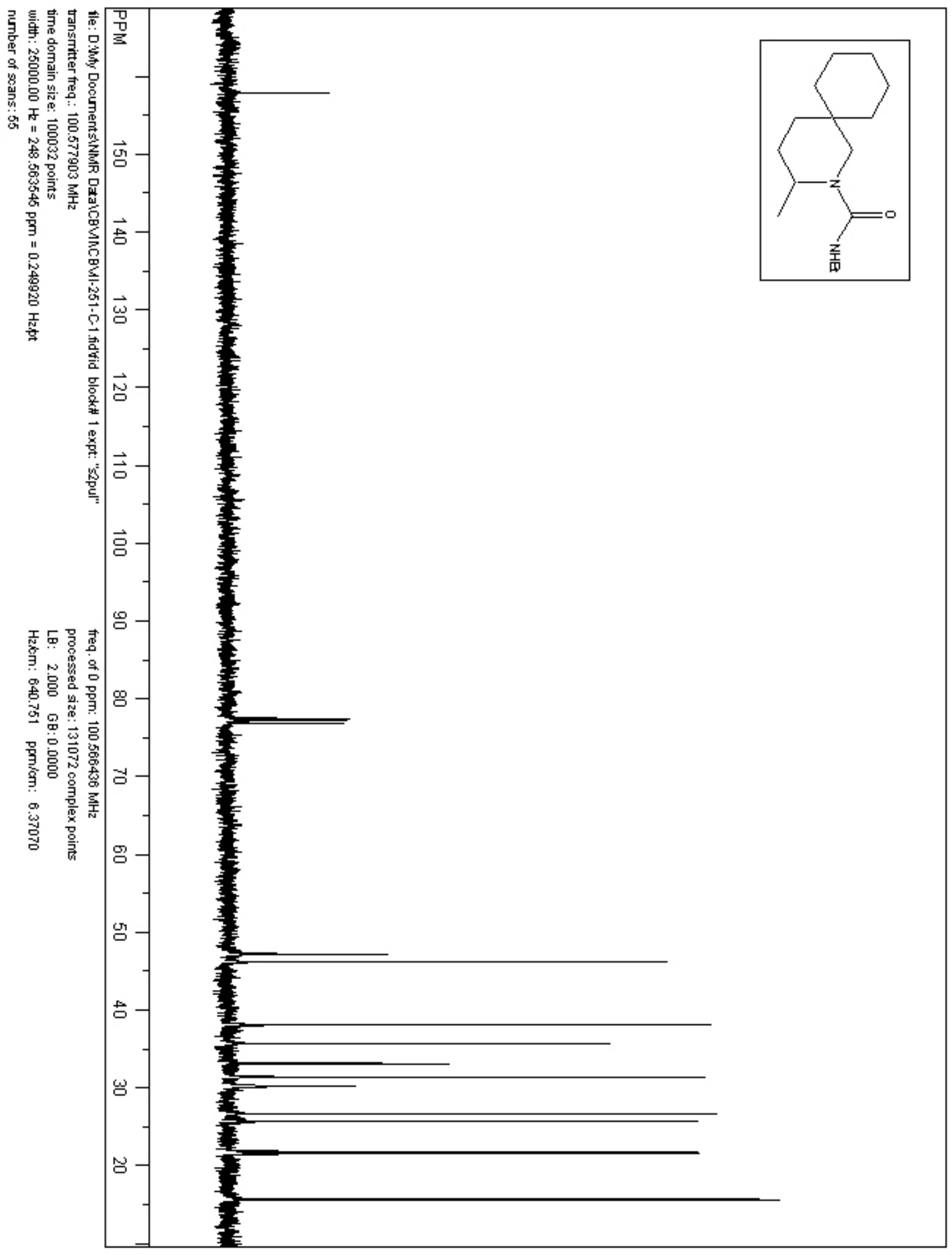




\section{References.}

S1) Bender, C. F.; Widenhoefer, R. A. J. Am. Chem. Soc. 2005, 127, 1070.

S2) Pour, M.; Špulák, M.; Balšánek, V.; Kuneš, J.; Kubanová, P.; Buchta, V. Biorg. \& Med. Chem. 2003, 11, 2843.

S3) Han, X.; Widenhoefer, R. A. Angew. Chem. Int. Ed. 2006, 45, 1747.

S4) Bender, C. F.; Widenhoefer, R. A. Chem. Commun. 2006, DOI: 10.1039/b608638a.

S5) de Frémont, P.; Scott, N. M.; Stevens, E. D.; Nolan, S. P. Organometallics 2005, 24, 2411.

S6) El Samii, Z. K. M.; Al Ashmawy, M. I.; Mellor, J. M. J. Chem. Soc., Perkin Trans. 1 1988, 2517. 